\title{
Cryptonite - A Programmable Crypto Processor Architecture For High-Bandwidth Applications
}

Rainer Buchty 



\author{
Institut für Informatik \\ der Technischen Universität München
}

Lehrstuhl für Rechnertechnik und

Rechnerorganisation

\title{
Cryptonite - A Programmable Crypto Processor Architecture For High-Bandwidth Applications

\author{
Rainer Buchty
}

Vollständiger Abdruck der von der Fakultät für Informatik der Technischen Universität München zur Erlangung des akademischen Grades eines

Doktors der Naturwissenschaften (Dr. rer. nat.)

genehmigten Dissertation.

Vorsitzender: $\quad$ Univ.-Prof. Dr. Hans Michael Gerndt

Prüfer der Dissertation:

1. Univ.-Prof. Dr. Arndt Bode

2. Univ.-Prof. Dr. Eike Jessen, em.

Die Dissertation wurde am 16. September 2002 bei der Technischen Universität München eingereicht und durch die Fakultät für Informatik am 27. November 2002 angenommen. 



\section{Cryptonite - A Programmable Crypto Processor Architecture For High-Bandwidth Applications}

Rainer Buchty 



\section{$\overline{\text { Abstract }}$}

Cryptography was and still is one of the most interesting fields in Computer Science-related research. Where its origin lies in military and governmental use, today cryptography is widely used in everyday life. Cryptography secures communication between smart cards and card readers. It scrambles transmissions between DECT telephones and their base stations, it even entered the living rooms through digital Pay TV channels which use cryptographic methods to make sure that only their subscribers can watch their transmissions - and only what they have payed for. Similarly, each DVD player contains cryptographic techniques which were implemented to prevent unauthorized copying and playback.

Also for network infrastructures, company intranets, or the global internet with its shared resources, cryptography is essential to secure transmitted data against snooping. This is especially vital to so called virtual private networks (VPNs), where a (virtually) private network is spanned using shared network resources meaning that although a shared medium is used, the spanned network behaves like a private network in terms of data security and connectivity. Even the underlying physical network infrastructure is hidden.

One of the biggest problems with modern cryptography is data throughput. Combined video/audio data as on DVDs or broadcasted via Pay TV stations easily needs - depending on the quality -2 to $10 \mathrm{MBit} / \mathrm{s}$ and more which means that networks carrying this data have to be able to provide at least the same bandwidth: A smaller bandwidth would lead to visible and audible artifacts.

Such applications require network devices which are not only capable of transporting the incoming data stream but also encrypt or decrypt them without becoming a bottleneck. For this reason, a number of dedicated hardware 
solutions exist which support one or more crypto algorithms. Naturally, such solutions do not allow to change the supported algorithms. Change of algorithm automatically means change of hardware; similarly, supporting a number of algorithms requires to have several of dedicated architectures each supporting one algorithm.

A programmable architecture is a solution to this dilemma. General purpose processors seem to be the ideal candidates for this task, however, these did not supply the needed computation power in the past - and today, where they do, they need too much electric power and produce too much heat. Also, it is not economically sensible to use processors worth several hundred Euro but using only a fraction of their potential.

In this thesis a programmable architecture dedicated to typical needs of cryptographic algorithms is presented. Starting with the analysis of several major cryptographic algorithms key parameters and hardware requirements were identified. Based on these, an architecture was designed which not only suits these requirements but also satisfies economic factors like cost of production and power consumption. A number of algorithms were implemented on an architectural simulator and dedicated parts of the architecture were realized using VHDL to measure hardware parameters such as logic use and routability. These numbers were compared against existing solutions; the comparison has proven that a programmable architecture like the one proposed within this work can achieve performance similar to or even better than existing dedicated hardware solutions while still retaining modest hardware requirements. 


\section{Acknowledgements}

This thesis would not have been possible without the support of many people. First of all, I would like to thank my advisor Professor Dr. Arndt Bode for the great freedom he gave me to conduct my research and the excellent research environment at LRR-TUM. I also want to express my gratitude to my co-referee Professor Dr. Eike Jessen for his valuable comments and hints.

The head of the Architecture Group, Dr. Wolfgang Karl, deserves credit for supporting my work. I want to especially thank him for making it possible for me to attend certain conferences which opened up more than fruitful opportunities.

Here, I would especially express my gratitude to Dr. Nevin Heintze (Agere Systems) for arranging an internship, and his colleague Dr. Dino Oliva (Agere Systems). Both of them deserve credits for their invaluable input and ongoing support. I further want to thank Ed Walters (Agere Systems) for taking the time to review my thesis so that it now will look not too awkward to native English speakers.

The internship would not have been possible without Dr.Tor Jeremiasson (Texas Instruments), who made contact between between Nevin Heintze and me on MICRO'33. Lastly, I want to thank Keith Bauer for providing a comfortable home during my stay in New Jersey.

Furthermore, I thank all of my former and current colleagues at LRRTUM, in particular Georg Acher and Deti Fliegl, for valuable technical and non-technical discussions and the great time we had and still have. Deti deserves further credits for administering our firewalled Intranet network infrastructure and providing a reliable and secure working environment. Besides, I want to thank my former project mates of the EPP and HotSwap projects, Jürgen Jeitner, Dr.Carsten Trinitis and Max Walter for yielding dis- 
cussions. I would also like to thank all my other colleagues for the friendly and familiar athmosphere at LRR-TUM which makes working there such a great pleasure.

Finally, I want to express my gratitude to my parents for their priceless support and sponsorship which made it possible for me to fully concentrate on my studies and research. I want to thank them especially for not allowing me to buy that video game back in the early 80 s but rather encouraging and sponsoring my interest in computers, computer science, and electrical engineering. My brother Martin I want to thank for sharing the interest in "ancient" electronics.

Last not least, I want to thank my love Nina for her great understanding and support.

Rainer Buchty

September 2002 


\section{Contents}

1 Introduction and Motivation 1

1.1 What is Cryptology? . . . . . . . . . . . . . . . 1

1.2 Use of Cryptology . . . . . . . . . . . . . . . 2

1.2.1 Secure Data Transfer . . . . . . . . . . . . 3

1.2.2 Authentication of Documents ......... 3

1.2.3 Data Distribution . . . . . . . . . . . . 4

1.2.4 Virtual Private Networks . . . . . . . . . . . . . . 4

1.2.5 Other Cryptological Applications ........ 5

1.3 Key Generation . . . . . . . . . . . . . . . . . 5

1.4 Need for speedy and secure cryptography . . . . . . . 6

1.5 Research Contribution . . . . . . . . . . . . . 8

1.6 Thesis Organization . . . . . . . . . . . . . . 9

2 Crypto Algorithms - Terms and Definitions 11

2.1 Symmetric and Asymmetric Algorithms . . . . . . . . . . 12

2.2 Modes of Operation . . . . . . . . . . . . . . . 13

2.2.1 Electronic Codebook Mode (ECB) . . . . . . . . . . 13

2.2.2 Cipher Block Chaining Mode (CBC) . . . . . . . . 14

2.2.3 Cipher Feedback Mode (CFB) . . . . . . . . . . . 16

2.2.4 Output Feedback Mode (OFB) . . . . . . . . . . . 17

2.2.5 Counter Mode $(\mathrm{CM}) \ldots \ldots$

2.3 Summary ...................... 18

3 Algorithm Discussion and Analysis $\quad 21$

3.1 Exploitable Parallelism . . . . . . . . . . . . . 21

3.2 Selection of Algorithms . . . . . . . . . . . . . 22

3.3 The Data Encryption Standard (DES) . . . . . . . . . 22 
3.3.1 Design of the DES Algorithm . . . . . . . . . . 24

3.3.2 Analysis of the DES Algorithm . . . . . . . . . 26

3.3.3 Proposed Computation Speed . . . . . . . . . . 28

3.4 Advanced Encryption Standard (AES / Rijndael) . . . . . . 30

3.4.1 Key Scheduling . . . . . . . . . . . . 31

3.4.2 Inner architecture of AES/Rijndael . . . . . . . . . 32

3.4.3 Implementational aspects of AES/Rijndael . . . . . 34

3.4 .4 Architectural Impact . . . . . . . . . . . . . . . . 34

3.5 The MD5 Message Digest Algorithm . . . . . . . . . . . 36

3.5.1 Analysis of the MD5 Algorithm . . . . . . . . 38

3.5.2 Similar Hash Algorithms . . . . . . . . . . . . 39

3.6 The Secure Hash Algorith (SHA) . . . . . . . . . . . . . 40

3.6.1 Proposed Computation Speed and Architectural Im-

pact .................... 41

3.7 The International Data Encryption Algorithm (IDEA) . . . . 43

3.7.1 Round Key Generation . . . . . . . . . . . . . . 44

3.7.2 Algorithm Analysis . . . . . . . . . . . . . . . 45

3.8 The RC6 ${ }^{T M}$ Block Cipher . . . . . . . . . . . . . . . 48

3.8 .1 Key Scheduling . . . . . . . . . . . 50

3.8.2 Algorithm Analysis . . . . . . . . . . . . . 50

3.9 Summary . . . . . . . . . . . . . 53

4 Architecture Impact resulting from Algorithm Analysis $\mathbf{5 5}$

4.1 Functional Units . . . . . . . . . . . . . . . 55

4.2 Register File . . . . . . . . . . . . . . . . . . . . . . . . . . . . 57

4.2.1 Register Size . . . . . . . . . . . . . 57

4.2.2 Number of Registers . . . . . . . . . . . . . . 58

4.3 Special Instructions . . . . . . . . . . . . . . . . . . . . . . . . 59

4.4 Summary ..................... 60

5 The Cryptonite Architecture $\quad 63$

5.1 Design Goals . . . . . . . . . . . . . . . . . 64

5.2 General Purpose Architectures - An Alternative? . . . . . . 65

5.3 Architecture overview . . . . . . . . . . . . 66

5.4 The Pipeline . . . . . . . . . . . . . . . . . . 68

5.5 Access from External Devices . . . . . . . . . . . . 69

5.6 The Control Unit . . . . . . . . . . . . . . . . 71 
5.6.1 Supplying immediate values . . . . . . . . . 71

5.6.2 Counters, looping, and conditional branching . . . . 73

5.6 .3 System Control . . . . . . . . . . . . . . . . . . 74

5.7 The Arithmetic Logical Unit . . . . . . . . . . . . . 74

5.7 .1 Overview .................. 74

5.7 .2 The Register File . . . . . . . . . . . . . . 74

5.7.3 The Arithmetic Unit (AU) . . . . . . . . . . 76

5.7 .4 The XOR Unit . . . . . . . . . . . . 80

5.7.5 Avoiding register pressure: Crosslinking the arithmetic units . . . . . . . . . . . . 80

5.8 The Memory Unit . . . . . . . . . . . . . . . . . . . . 81

5.8.1 Address Generation Unit . . . . . . . . . . . . . 81

5.8.2 Speeding up table lookups: S-Box support . . . . . . 84

5.8 .3 The Data Input/Output Unit . . . . . . . . . . . 85

5.8.4 The DES-specific unit . . . . . . . . . . . . 87

5.9 Summary . . . . . . . . . . . . . . . 90

6 Implementational Aspects $\quad 93$

6.1 Software Implementation . . . . . . . . . . . . . . . 93

$6.1 .1 \quad$ AES/Rijndael . . . . . . . . . . . . . . . 94

6.1 .2 DES and 3DES . . . . . . . . . . . 95

6.1 .3 IDEA . . . . . . . . . . . . 95

6.1 .4 RC6 . . . . . . . . . . . . . 95

6.1 .5 MD5 and SHA-1 . . . . . . . . . . . 95

6.2 Hardware Implementation . . . . . . . . . . . . 96

6.2.1 Technology Requirements . . . . . . . . . . . 96

6.2.2 Implementation Results _. . . . . . . . . . . . 97

6.3 Summary . . . . . . . . . . . . . . . . 99

7 Comparison of CRYPTONITE against Existing Solutions $\quad 101$

7.1 Hardware Solutions \& IP Cores . . . . . . . . . . . . . . 102

7.1.1 Amphion Semiconductor Ltd. . . . . . . . . . . . 102

7.1.2 Broadcom Corporation . . . . . . . . . . . 105

7.1.3 Corrent Corporation . . . . . . . . . . 106

$7.1 .4 \mathrm{Hi} / \mathrm{fn}$, Inc. . . . . . . . . . . . . . . . 106

7.1 .5 NetOctave, Inc. . . . . . . . . . . . . . . . . 109

7.1 .6 OpenCores.org ................ . . . . . . . . 
7.1.7 Secucore Consulting Services . . . . . . . . . . 113

7.1 .8 Zyfer, Inc. . . . . . . . . . . . . . . . . . . . . . . 113

7.2 Programmable Solutions . . . . . . . . . . . . . . . . . 114

7.2 .1 CryptoManiac . . . . . . . . . . . . . . . . . 114

7.2.2 PLD001 Cryptoprocessor . . . . . . . . . . . . . 119

7.3 Algorithm Performance Comparison . . . . . . . . . . 121

7.3.1 AES-128/128 Performance . . . . . . . . . . . 122

7.3.2 DES Performance . . . . . . . . . . . . . . . . 123

7.3.3 3DES Performance . . . . . . . . . . . . . . . . 124

7.3.4 IDEA Performance . . . . . . . . . . . . . . . . . . 125

7.3.5 RC6 Performance . . . . . . . . . . . . . . . . 125

7.3.6 MD5 Performance . . . . . . . . . . . . . . 126

7.3.7 SHA-1 Performance . . . . . . . . . . . . 128

7.4 Summary . . . . . . . . . . . . . . . . . . . . . . 129

8 Conclusion and Summary 131

9 Future Work 135

9.1 Software Development . . . . . . . . . . . . . . . 135

9.1.1 CRyptonite Assembler . . . . . . . . . . . . . 136

9.1.2 C-Compiler for the CRYPTONITE Architecture . . 136

9.2 Hardware Development . . . . . . . . . . . . . . . 136

9.2.1 Single-Core CRYPTONITE . . . . . . . . . . . . 136

9.2.2 Multi-Core CRyptonite . . . . . . . . . . . . 137

9.3 Use of CRYPTONITE . . . . . . . . . . . . . . . . . . . . . 138

9.3.1 Research \& Development . . . . . . . . . . . . 138

9.3.2 Conditional Access Systems . . . . . . . . . . . 138

9.3 .3 VPN Devices . . . . . . . . . . . . . . . . . 138

A Instruction Set 141

A.1 Immediate Values . . . . . . . . . . . . . . . . . 141

A.2 Control Instructions . . . . . . . . . . . . . . . . . 142

A.3 Memory Instructions $\ldots \ldots \ldots$

A.4 Arithmetic Instructions $\ldots \ldots \ldots$

A.5 XOR Instructions $\ldots \ldots \ldots \ldots$ 
B Instruction Word Format 149

B.1 Instruction Word . . . . . . . . . . . . . . . . . 149

B.2 Support of Immediate Values . . . . . . . . . . . . 150

B.3 Control Unit Command Encoding . . . . . . . . . . . . . 151

B.4 ALU-common Control Encoding . . . . . . . . . . . 151

B.5 XOR Unit Command Encoding . . . . . . . . . . . . 153

B.6 Arithmetic Unit Command Encoding . . . . . . . . . . . . 153

B.7 Memory Unit Command Encoding . . . . . . . . . . . . 155

C Proposed Assembly Language Format 157

C.1 Register Naming Convention . . . . . . . . . . . . . . 157

C.2 Assembly Language Format . . . . . . . . . . . . 157

C.3 Programming Examples . . . . . . . . . . . . . . . . . 162

C.3.1 Example 1: Initialization . . . . . . . . . . . . 162

C.3.2 Example 2: Update key \& data, return processed data 162

C.3.3 Example 3: DES Implementation . . . . . . . . . 163

$\begin{array}{ll}\text { D Glossary } & 165\end{array}$

$\begin{array}{ll}\text { Bibliography } & 169\end{array}$

$\begin{array}{ll}\text { Index } & 181\end{array}$ 


\section{Figures}

2.1 Encryption and Decryption using CBC . . . . . . . . 15

2.2 Cipher Feedback Mode (CFB) . . . . . . . . . . . . 17

2.3 Output Feedback Mode (OFB) $\ldots \ldots \ldots$

3.1 Workflow of the DES Algorithm . . . . . . . . . 23

3.2 A single DES round . . . . . . . . . . . . . . 24

3.3 The DES S-Box . . . . . . . . . . . . . 25

3.4 Triple-DES . . . . . . . . . . . . . 26

3.5 AES Encryption . . . . . . . . . . . . . . . 31

3.6 AES Decryption . . . . . . . . . . . . . . . . . . 32

3.7 Message Padding for MD5 . . . . . . . . . . . 36

3.8 Workflow of the MD5 Algorithm . . . . . . . . . . . 37

3.9 A single MD5 round . . . . . . . . . . . . . . . 37

3.10 MD5 Data Dependencies . . . . . . . . . . . . . . 38

3.11 The MD5 non-linear functions F, G, H, and I . . . . . . . 39

3.12 SHA Data Dependencies . . . . . . . . . . . . 41

3.13 The SHA non-linear functions for rounds $1,2 / 4$, and $3 \ldots 42$

3.14 Workflow of IDEA . . . . . . . . . . . . . . . . . . 44

3.15 A single round of IDEA . . . . . . . . . . . . . . 45

3.16 Data flow within an IDEA round . . . . . . . . . . 47

3.17 Workflow of the RC6 ${ }^{T M}$ Block Cipher . . . . . . . . . . . 49

3.18 Data flow within one $\mathrm{RC}^{T M}$ round $\ldots \ldots \ldots 1$

5.1 Overview over the CRYPTONITE architecture . . . . . . 67

5.2 The External Access Unit . . . . . . . . . . . . . . 70

5.3 Overview over CRYPTONITE's Control Unit . . . . . . . . 72

5.4 Overview over CRYPTONITE's ALU . . . . . . . . . . 75

5.5 Detail of the arithmetic unit . . . . . . . . . 78 
5.6 The Address Generation Unit . . . . . . . . . . . . . . . . 82

5.7 Direct and indexed address generation . . . . . . . . 84

5.8 Modulo addressing . . . . . . . . . . . . . . . . 84

5.9 S-Box address generation . . . . . . . . . . . . . . 85

5.10 Data I/O from embedded SRAM . . . . . . . . . . . . 86

5.11 Detail of CRYPTONITE's DES Unit . . . . . . . . . . . . 89

7.1 CryptoManiac Architecture Overview . . . . . . . . . . 115

7.2 CryptoManiac's functional unit . . . . . . . . . . 116

7.3 IDEA configuration . . . . . . . . . . . . . . . 119

7.4 Calculation Mode . . . . . . . . . . . . . . . . 120

7.5 Data flow within the PLD001 ALU . . . . . . . . . . 120

7.6 PLD001 ALU control structure . . . . . . . . . . . . . 121

7.7 AES-128/128 Performance Comparison . . . . . . . . . 122

7.8 DES Performance Comparison . . . . . . . . . . . . . . . 124

7.9 3DES Performance Comparison . . . . . . . . . . . 125

7.10 IDEA Performance Comparison . . . . . . . . . . 126

7.11 RC6 Performance Comparison . . . . . . . . . . . . . . . 127

7.12 MD5 Performance Comparison . . . . . . . . . . . . . . . 128

7.13 SHA-1 Performance Comparison . . . . . . . . . . . . 129 
3.1 Naive DES Implementation . . . . . . . . . . . . . . 27

3.2 A faster DES Implementation . . . . . . . . . . . . . . . 27

3.3 An even faster DES implementation (excluding key generation) . . . . . . . . . . . . . 27

3.4 Ideal DES implementation including Round Key generation 27

3.5 Architectural requirements for efficient (3)DES implementation ....................... 29

3.6 Number of Rounds as a function of block and key size . . . 30

3.7 Shift offsets for different block lengths . . . . . . . . . . . . 33

3.8 Architectural requirements for efficient AES/Rijndael implementation ................. 35

3.9 Chaining Variables Init Values . . . . . . . . . . . . 36

3.10 The non-linear functions . . . . . . . . . . . 38

3.11 MD4 non-linear functions . . . . . . . . . . . . . 40

3.12 The SHA non-linear functions . . . . . . . . . . . 41

3.13 Architectural requirements for efficient MD5 and SHA implementation ................. 42

3.14 IDEA sub-keys used for encryption and decryption . . . . . 46

3.15 Architectural requirements for efficient IDEA implementation 48

3.16 RC6 Basic Operations . . . . . . . . . . . . . . . . 50

3.17 Speed estimations for RC6 on an ideal architecture . . . . . 52

3.18 Architectural requirements for efficient $\mathrm{RC}^{T M}$ implementation ..................... 52

4.1 Operations employed in analyzed algorithms . . . . . . . . 56

4.2 Register Size Control . . . . . . . . . . . . . . . . . . . . 59

5.1 Pipeline Stages of the CRYPTONITE Architecture . . . . . 68 
5.2 Interrupt Cycles . . . . . . . . . . . . . 71

5.3 The upper64 and lower64 functions . . . . . . . . . 77

5.4 The swap functions . . . . . . . . . . . . . . . 79

5.5 The fold operations . . . . . . . . . . . . . . . . 79

5.6 Addressing modes supported by CRYPTONITE's AGU . . . . 83

5.7 Special functions for non-monolithic DES implementation . 87

5.8 Special functions for pipelined DES implementation . . . 88

6.1 CRYPTONite Performance Data for Selected Algorithms . . 94

6.2 Synthesis Results on Xilinx Virtex-IIp FPGA family . . . . 98

7.1 CS52xx AES Core Performance . . . . . . . . . . . 103

7.2 Technology and Performance Comparison for Amphion Cores 104

7.3 Broadcom BCM58xx performance regarding packet size . . 107

7.4 Broadcom BCM58xx performance data . . . . . . . . 108

7.5 Performance Data of Hifn Processors . . . . . . . . . . . . 110

7.6 Algorithm cycle counts computed from given performance data . . . . . . . . . . . . . . . . 110

7.7 Performance Data for NetOctave SSL and IPsec Processors . 111

7.8 OpenCores DES Performance Data . . . . . . . . . . . . . . 112

7.9 SecuCore Performance Data . . . . . . . . . . . . . 113

7.10 Kernel loop cycle counts per round . . . . . . . . . . . 117

7.11 Timing and Area Results for CryptoManiac . . . . . . . . 118

7.12 Estimated Throughput for CryptoManiac running at $360 \mathrm{MHz} 118$

7.13 PLD001 Performance Data . . . . . . . . . . . . 121

7.14 AES-128/128 Performance Comparison . . . . . . . . . . . . 122

7.15 DES Performance Comparison . . . . . . . . . . . . 123

7.16 3DES Performance Comparison . . . . . . . . . 124

7.17 IDEA Performance Comparison . . . . . . . . . . . . 125

7.18 RC6 Performance Comparison . . . . . . . . . . . . . 126

7.19 MD5 Performance Comparison . . . . . . . . . . . . . . 127

7.20 SHA-1 Performance Comparison . . . . . . . . . . . 128

A.1 Immediate Load Instruction (applies to Control Unit) . . . 142

A.2 Control Instructions . . . . . . . . . . . . . . 143

A.3 Memory-to-Register and Register-to-Memory Instructions . 144

A.4 DES-specific Instructions . . . . . . . . . . . . . . . 145

A.5 ALU Instructions . . . . . . . . . . . . . . . . . 146 

A.6 XOR Unit instructions $\ldots \ldots \ldots$. . . . . . . . . . . . . .
B.1 CRYPtonite Instruction Word Format . . . . . . . . 150
B.2 Register Adresses . . . . . . . . . . . . . . . . . . . . 150
B.3 Register Size Selection Values . . . . . . . . . . 151
B.4 Control Unit Instruction Format . . . . . . . . . . . 152
B.5 ALU-common Configuration Pattern . . . . . . . . . . . 152
B.6 XOR Unit Instruction Format . . . . . . . . . . . . . 153
B.7 Arithmetic Unit Instruction Format . . . . . . . . . . . 154
B.8 Memory Unit Instruction Format . . . . . . . . . . . 155
C.1 Register Naming Conventions _ . . . . . . . . . . 158
C.2 VLIW-style Assembly Instruction . . . . . . . . . . 158
C.3 BNF Notation of a CRYPTONITE Mnemonic . . . . . . . . 159
C.4 BNF Notation of a CRYPTONITE Instruction . . . . . . . . . 159
C.5 BNF Notation of Primitives and Composita . . . . . . . . 160
C.6 BNF Notation of Labels and Constant Declarations . . . . . 161 


\begin{tabular}{lc}
\hline \hline+2 & \\
& \\
\hline \hline
\end{tabular}

\section{Introduction and Motivation}

\subsection{What is Cryptology?}

When talking about cryptology in general the terms cryptology and cryptography are often used very loosely. To avoid confusion this document follows the definitions given by the American National Standard for Telecommunication (ANST) as part of the Alliance for Telecommuncations Industry Solutions (ATIS) [6] which are cited below.

Cryptology is both "the science that deals with hidden, disguised or encrypted communications" and "the field encompassing cryptography and cryptanalysis” [123].

The term cryptography describes "the art or science concerning the principles, means and methods for rendering plain information unintelligible, and for restoring encrypted information to intelligible form" [122]. It also refers to this term as "the branch of cryptology that treats the principles, means, and methods of designing and using cryptosystems" [122].

Measuring the quality of a cryptographic method is done by means of cryptanalysis, namely "operations performed in converting encrypted messages to plain text without initial knowledge of the crypto algorithm and/or 
key employed in the encryption" [121]. This definition can be condensed to "study of encrypted texts" [121] ${ }^{1}$.

\subsection{Use of Cryptology}

Today, cryptology is widely used and has already pervaded everyday life in many ways. So called smart cards use encryption for securing the communication between its host - for example a public telephone - and the smart card processor. Digital pay TV stations scramble their signals in a way which allows only the subscribers to descramble the transmissions. ID cards attached to a PC are used for authentication and digital subscription.

As these examples show, the role of cryptography is manifold; cryptographic algorithms perform the following tasks which directly relate to the above examples:

- Securing a point-to-point connection making it impossible to snoop into the communication to gain information about both, the transmitted data and the protocol used for data transmission. Two flavours of reversible data encryption exist which are symmetric and asymmetric encryption. In the first case, the same key is used for encryption and decryption; in the second case different ones are used. This will be explained in more detail when discussing asymmetric algorithms in Section 2.1.

- Securing a one-to-many broadcast ensuring that a group of individual recipients (and only this group) is able to decipher the transmitted data. This requires special encryption techniques which allow the use of group key(s) for encryption where the decryption is done using individual (subscriber, for instance) keys.

- Authentication of data; this can mean either proving the integrity of the transmitted data by providing some kind of checksum or authenticating the origin of a message. Data integrity is ensured by performing a one-way hash function over the received data and comparing the

\footnotetext{
${ }^{1}$ Since this work focuses on the development of an efficient programmable architecture for crypto algorithms this work will not deal with cryptanalysis issues beyond introductory information to each algorithm.
} 
computed value against a reference value provided by the sender. A commonly known use of this technique is storage and verification of passwords under the various Unix and Unix-like operating systems which only store the hash value of the password instead of the password itself.

Sender authentication comes virtually for free when using asymmetric Public Key encryption techniques which will be introduced in Section 2.1.

\subsubsection{Secure Data Transfer}

This is the basic application of cryptography: Information has to be encrypted in a way that only the recipient (or a group of recipients as for the Pay TV example) can access the original, unencrypted data.

As mentioned in Section 1.2, two basic encryption methods exist: symmetric encryption and asymmetric encryption. In Section 2.1 these methods will be discussed more detailed showing how this class of algorithms can be used for digital signing of data.

Also, the way data is transmitted needs to be taken into account. Algorithms can either work on data chunks of same size (block ciphers) or continuous data streams (stream ciphers). This will be discussed in Chapter 2 which will also include an introduction into chained ciphering where preceding data is included into the encryption process of following data.

\subsubsection{Authentication of Documents}

Securing data transfer does only guarantee (to a certain degree) that no unauthorized person is able to read the encrypted message. However, it does not prove authenticity. Besides securing against so-called snooping, the recipient has also to be sure that the transmitted data is authentic and does indeed originate from the denoted sender.

One example might be online banking: The bank has to ensure that outgoing money transfers are initiated by the account holder only. Just using login and password for authentification would be highly insufficient; thus, every transaction is not only secured by the Personal Identification Number (PIN) but also by a per-transaction individual Transaction Number (TAN). 
Used TANs are stored in a blacklist ensuring that each TAN can be used only one time.

Another example comes from civil service: Recent approaches target an entire "electronic service" allowing citizens to fill out necessary forms for services like passport renewal, announcing movements, or car licensing on-line. Security for this systems needs to be very tight to prevent possible identity frauds. Both, the abuse of another person's account, and pretending a different identity must be prohibited. Furthermore, all transfers must be encrypted in a way that only the denoted recipient is able to decrypt the transmissions to avoid disclosure of sensitive or personal data.

The already mentioned asymmetric encryption also known as public key encryption offers a solution to this problem.

\subsubsection{Data Distribution}

In certain cases it wanted to address a group of recipients rather than allow only one single recipient to decrypt an encrypted message. The most common use of this technique is Pay TV where all subscribers of a channel have to be able to decrypt the scrambled TV transmission. It is also applicable to other means of Digital Rights Management (DRM) where access to information is restricted to a set of subscribers, possibly with varying access rights.

Since this is mainly a mathematical problem dealing with key generation algorithms further discussion of this field are omitted from this work. An introduction into the field of DRM can be found in [64] and [77].

\subsubsection{Virtual Private Networks}

Instead of expensively creating their own physical network many companies use existing network infrastructures. These can be either private networks shared among several companies but also public ones like the Internet. In either case sensitive corporate data needs to be secured from external observation - despite the shared medium the transported data still needs to be kept private, thus the term Virtual Private Network (VPN).

Several software solutions exist which create a secured so-called tunnel between two endpoints spawning a virtual network structure over an existing 
network. One of the most common software packages used is the IPSEC system as incorporated in the FreeS/WAN project [124].

It is not uncommon that two VPN nodes connecting entire internal networks (hereafter referred to as intranets as opposed to the Internet) need to process an enormous workload of several $100 \mathrm{MBit} / \mathrm{s}$. The connecting nodes have to cope with these data streams and process them immediately so they do not become a bottleneck and slow down the network interconnection. This would eventually disrupt ongoing real-time data streams like video conferencing, for example.

\subsubsection{Other Cryptological Applications}

Plenty of additional applications for cryptological algorithms exist such as electronic voting: Here it must be ensured that every person is able to vote only one time, but on the other hand the identity of that person has to be kept secret. For a detailed introduction into secret voting the interested reader is referred to pages 149 to 159 of [106]. The same source also lists many other applications such as distributed secret sharing where a message is split up into a number of pieces where each piece can be proven to be authentic but the message can only be decrypted when all pieces are put together. This encryption method is ideal for companies selling a unique product such as the Coca Cola company which obviously have to ensure that never a single person knows the receipe for their soft drinks.

Other examples of cryptological applications are immediate exchange of secrets, immediate signing of contracts, exchanging data through a trusted third party like a notary, or approving data by a third person. All of these are interesting mathematical questions concerning algorithm construction and key generation; the interested reader might find an absorbing introduction into the various applications of cryptology in [106]. For this work, however, this short introduction should be sufficient.

\subsection{Key Generation}

Keys needed for asymmetric cryptographical algorithms (see Section 2.1) are based on huge prime numbers. To find such numbers fast is still one of the greatest challenges in cryptology. An introduction into this field would 
surely go beyond the focus of this work; the interested reader will find an overview over the various methods of prime number generation and proving primality in [29] and [38].

For about 15 years polynomial methods based on elliptic curve equations $[12,129]$ have been proven to be among the most efficient methods; for that reason a number of elliptic curves are recommended by the National Institute of Standards and Technology (NIST) [78] and numerous work was done on the field of implementing so-called elliptic curve processors, both in hardware [98, 71, 75, 76, 87, 130, 117] and software [14, 49, 111, 88]. However, the problem of generating large prime numbers is quite different from the cryptographic algorithms used for en- or decryption, which is why key generation will not be included in this work.

Whenever the term key generation is used it refers to round key generation $^{2}$. The term round denotes one iteration of the algorithm's inner loop. For example the DES algorithm consists of 16 rounds as explained in Section 3.3. Unlike the cipher key, which has been computed by aforementioned methods, the round key is generated using independent rules as defined within the chosen cryptographic algorithm. Roughly said, it is a special permutation based on the cipher key and an integral part of its referring cryptographic algorithm. The methods of key generation will be discussed along with the respective cipher algorithms in Sections 3.3 and 3.4.

\subsection{Need for speedy and secure cryptography}

The use of cryptography is an emerging market. The growing demand for cryptography arises from the desire to secure networks and data against potential intruders and also the marketing of intellectual property.

In the past the data media itself was adequate copy protection: it is impossible (or better: economically not sensible) to create an exact copy of a book or a magazine, the same goes for audio vinyl records or movies on VHS tape. Whenever one tries to copy these media by means available at reasonable prices the copy is of lower quality than the original. The digital age has changed this dramatically: Data stored on digital media can be copied without loss of quality and at nearly no cost.

\footnotetext{
${ }^{2}$ Another term for round key generation often found in literature is key setup.
} 
With increasing network performance and growing storage capacities the type of transmitted data changed. Whereas text data such as HTML pages usually does not require much bandwidth or, in case of text documents, does not have any real-time demands, requirements for so-called multimedia data like streaming video and audio are quite different. Audio streams in CD quality usually have a bit rate of $128 \mathrm{kBit} / \mathrm{s}$ to $256 \mathrm{kBit} / \mathrm{s}$ which is already twice to four times the data rate provided by a single ISDN data channel. Even more resource-greedy is video transmission which can easily use $10 \mathrm{MBit} / \mathrm{s}$ - an amount which was quite sufficient for entire companies' internal networks (intranets) just a decade ago.

This shows that especially for securing multimedia streams (usually on a per-user base as being used for pay-per-view services) a huge amount of data needs to be encrypted with minimum latency and appropriate throughput to meet the strict real-time requirements such streams have.

But it is not only multimedia which has high demands for bandwidth and data throughput. As mentioned before, companies often connect their local intranets over the internet using secure tunnels. These VPNs have to transport the entire communication between physically separated local nets. Companies with great network bandwidth demands use $155 \mathrm{MBit} / \mathrm{s}$ to $622 \mathrm{MBit} / \mathrm{s}$ lines to realize the intranet linking already requiring dedicated network routers. En- or decrypting these enormous data streams in realtime requires further hardware since this is far beyond the capabilities of any PC-based software solution.

Such solutions already exist. Back in the 1980s dedicated DES processors were built; today the first chips targeted towards the Advanced Encryption Standard (AES) have hit the market. However, these processors can only perform one single cryptographic algorithm - even worse with AES where common hardware solutions usually support only one out of 9 possible configurations as shown in Chapter 7.

Interestingly, until now almost no universally programmable crypto processors are known to the public. The available solutions, mostly for smartcard based cryptography, are designed for low-bandwith applications like exchange of keys or challenge-response operations. As of today, only one freely programmable and algorithm-independent crypto processor has shown up in literature which is capable of processing at least medium bandwidth data [131]. In addition, a microprogrammable solution [67] exists 
which - although it was tailored towards the IDEA algorithm - is not necessarily restricted to that algorithm.

\subsection{Research Contribution}

The research contribution of this work is the development of a novel crypto processor architecture targeting high-bandwidth applications. It is based on exhaustive algorithm analysis with respect to parallelism, memory, and register use as well as support of non-standard functions. Currently existing solutions are mainly hardwired logic which means that these chips perform only one or a very limited set of algorithms. In contrast, the proposed CRYPTONITE architecture is fully programmable and is not tailored towards a single algorithm: The flexibility of the CRYPTONITE architecture is not achieved by a collection of dedicated processing units where each unit is responsible for a single algorithm; instead, the computation units are designed to be as general as possible ${ }^{3}$.

To determine the proper base architecture a set of crypto algorithms was selected and analyzed to determine the architectural needs of each algorithm. This analysis started with a straight-forward implementation of the selected algorithms to firstly determine exploitable parallelism by analysis of data and control flow. A more fine-grained analysis was then performed to determine the number and type of functional units as well as supporting instructions. The data was collected and processed to determine an architecture which would be suitable for all investigated algorithms. Based on current and expected usage some algorithms were weighted over others (AES/DES).

The aim of this work was to create a programmable architecture suitable for a broad range of cryptographic algorithms used for encryption, decryption and fingerprinting (authentication) of data with respect to processing speed and manufacturing costs. To achieve this the algorithms were implemented in many ways to determine the optimal compromise between speed and complexity of the architecture as presented through Chapters 3 to 5; Section 5.2 lists reasons why a dedicated crypto architecture was preferred over using a high-performance general purpose processor.

\footnotetext{
${ }^{3}$ In case of DES, however, it was vital to provide a specialized DES unit to achieve high throughput. See Section 5.8.4 for further discussion of this topic.
} 
Performance numbers as listed in Chapter 6 have been calculated based on software simulation and VHDL model synthesis. For software simulation a cycle-exact simulator was written to allow implementation and testing of selected algorithms. To determine hardware requirements selected parts of the CRYPTONITE architecture were modelled using VHDL; these models were then synthesized and fitted into the Xilinx Virtex-IIpro FPGA family.

Depending on the algorithm, the CRYPTONITE architecture presented within this work shows a raw crypto performance of 250 to $780 \mathrm{MBit} / \mathrm{s}$ including round key calculation. Is is remarked that usual software and also some hardware implementations do not include round key generation embedded into the ongoing encryption or decryption process but rather operate on precomputed round keys. The achieved throughput is not only in the range of comparable dedicated hardware solutions but even outperforms a number of these as shown in Chapter 7. In addition, the proposed architecture shows also superior performance within the sparse field of truly programmable solutions. It shall be remarked again that the algorithm implementations as realized on the CRYPTONITE architecture include round key generation.

This very promising performance is resulting from the general architectural concept which was tailored towards the demands of typical cryptographic algorithms and a special memory access technique, the S-Box lookup as explained in Section 5.8.1, and specialized arithmetic operation supporting certain algorithms as listed in Section 5.7.3.1.

\subsection{Thesis Organization}

The thesis is organized as follows: Chapter 2 will give an overview over the field of cryptology and basic definitions such as operation modes of crypto algorithms. After this introductionary part, the following Chapter 3 will present selected algorithms used for architectural demand analysis together with their relevance within the field of crypto systems.

Based on this analysis, the architectural requirements are summed up in Chapter 4 and the resulting architecture is presented in Chapter 5. Implementational aspects of both, the software and hardware side, are shown in Chapter 6 which also gives performance estimations based on software sim- 
ulation and VHDL model synthesis. This topic is completed with Chapter 7 which compares the proposed architecture against existing solutions.

The thesis is closed with Chapter 8 giving conclusion and summary followed by Chapter 9 offering a brief outlook on future work and closes with some final remarks. It is finished with an overview of the instruction set in Appendix A and a description of the instruction word format described in Appendix B. Finally, the Assembly Language Format for CRYPTONITE is presented in Appendix $\mathrm{C}$ together with sample code. 


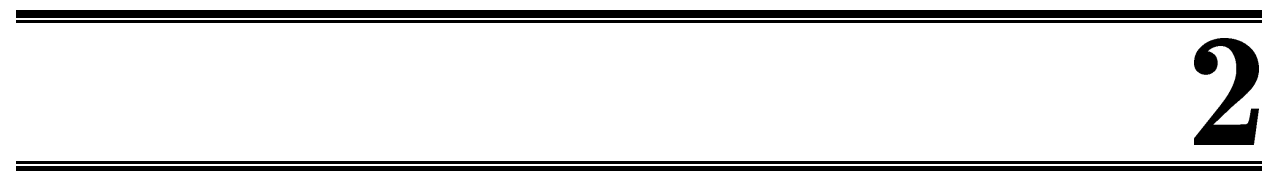

\section{Crypto Algorithms - Terms and Definitions}

This chapter will elaborate on certain crypto algorithms: Those used for reversable encryption, which hereafter will be called cipher algorithms, and those for the generation of fingerprints or hashes, the hash algorithms.

Cipher algorithms are bijective mathematical constructs: They allow encryption of a plain text message into cipher text but also the reverse operation, decryption of a cipher text into the original plain text message. The group of hash algorithms, however, is injective. For every input message they create a distinct hash code of a typical size (512-bit for MD5) which then, for instance, can be used for authentication of messages.Traditionally, Cipher algorithms can be as simple as the Caesar chiffre which is just a permutation of the alphabet by a certain permutation factor. Instead, modern crypto systems make use of so-called cipher keys. These keys are derived from a special cipher key space which has to adhere to certain rules.

For systems which use the same keys for encryption and decryption equations 2.1 and 2.2 apply. Here, $E_{K}$ and $D_{K}$ represent the respecting encryption and decryption functions based on a key $K . M$ and $C$ denote original message and cipher text. The functions $E_{K}$ and $D_{K}$ are constructed to be the reverse functions of each other so that equation 2.3 is valid.

$$
\begin{array}{r}
C=E_{K}(M) \\
M=D_{K}(C) \\
M=D_{K}\left(C_{K}(M)\right)
\end{array}
$$


Algorithms following equations 2.1 to 2.3 use the same key for encryption and decryption. Apart from these, a second class of algorithms exists. These algorithms use different keys $K_{e}$ and $K_{d}$ for encryption and decryption instead of a uniform cipher key $K$. For these algorithms equations 2.4 to 2.6 apply.

$$
\begin{array}{r}
C=E_{K_{e}}(M) \\
M=D_{K_{d}}(C) \\
M=D_{K_{d}}\left(C_{K_{e}}(M)\right)
\end{array}
$$

\subsection{Symmetric and Asymmetric Algorithms}

As just discussed, cipher algorithms can be divided into two distinct classes which are symmetric and asymmetric (or public key) algorithms.

Symmetric algorithms use the same key for encryption and decryption of a message as discussed in the previous section. Consequently, this common key has to be kept secret between the communication partners under all circumstances. The class of symmetric algorithms divides further into stream ciphers and block ciphers. As the name suggests, stream ciphers operate on a stream of data; depending on the context such a stream can be either be a stream of bits or bytes. Block ciphers, in contrast, operate on data chunks of certain size which means that a message distributes over a number of blocks. The size of these blocks are determined by the selected algorithm and algorithm configuration. In case of DES, which is the first standardized crypto algorithm, block size is 64-bit. Using certain operation modes as discussed in Section 2.2, block ciphers can also be applied to streaming data.

Asymmetric algorithms - more figuratively called public key algorithms - use different keys for encryption and decryption. One key is called public key and can be handed out to all communication partners without compromising security. The second key, however, must be kept private and is therefore called private key .

Public key algorithms are designed in a way that a message encrypted with someone's public key can only be decrypted with that person's private key - and vice versa. This makes it possible to encrypt a message in 
a way that it can be only deciphered by the addressee. Together with message authentication based on hash algorithms - which provide information about whether a data transmission was modified during transportation or not - public key algorithms ensure the authenticity of a message where the addressee can be sure that the message originates from the denoted sender (provided by the public key algorithm) and that the message content has not been altered (provided by the hash algorithm).

\subsection{Modes of Operation}

When discussing cipher algorithms in the context of developing a suitable crypto architecture, both, the algorithm itself and its mode of operation must be taken into account. These operation modes influence the way an algorithm can be implemented and especially affect efficiency and possible exploitation of parallelism. Besides, certain modes of operation allow the use of block-based cipher algorithms for character-based data streams which, for instance, are used for remote terminal applications (remote shells).

Numerous operation modes and combinations of these exist which can be reduced to one of the "primitive" modes which are electronic codebook mode (ECB), cipher block chaining (CBC), cipher feedback mode (CFB), output feedback mode (OFB), and counter mode (CM). Some of these modes employ shift registers into which computation results are fed back; another group of operation modes uses sequence numbers instead of computation results and are therefore called counter modes. For modes being derived from these modes the dedicated reader is referred to [103].

For certain crypto algorithms various operation modes are specified; Sections 2.2.1 to 2.2.5 will shortly introduce these operation modes prior to discussing the examined cipher algorithms in detail. For a more detailed discussion on operating modes please refer to [105].

\subsubsection{Electronic Codebook Mode (ECB)}

ECB is a straightforward way to use block cipher: a plain text block is directly encoded into a cipher text block. Assuming the use of identical keys plain text blocks of same content will be encrypted into cipher text blocks of same content. This would allow the creation of a codebook or dictionary 
showing the correlation between plain and cipher text block. On one hand, this is only a theoretical consideration since with cipher algorithms working on n-bit blocks such a codebook would have $2^{n}$ entries, furthermore with a key size of $\mathrm{k}$ bit, $2^{k}$ of these codebooks would be needed. On the other hand a professional cryptanalyst would be able to attack this mode by creating such codebooks and do a statistical analysis: Recent experiments have shown that it indeed is possible to identify language and even the author by statistically analyzing the codebook of compression algorithms which use codebook methods similar to ECB-based encryption against known language parameters $[13,50]$.

Since plain text blocks can be processed independently, ECB ensures linear coarse-grained increase in throughput by simply increasing the number of computation units and distributing a message of arbitrary length (which has to be a multiple of the block size $n$, of course) evenly among all computation units present in the system. This is unlike the feedback modes discussed in the following sections which inherently inhibit this coarsegrained parallelism.

ECB is resistant against bit errors (bit value changes) since only the block containing one or more error bits is affected; there is no error propagation. However, ECB is very sensitive to synchronization errors: Once the synchronization is lost, for example by accidental bit insertion when receiving a encrypted message, the remaining message will be unreadable.

\subsubsection{Cipher Block Chaining Mode (CBC)}

With cipher block chaining the previously generated cipher text block block is used as another input value for the en- or decryption process. This means, that processing a single data block is not independent like in ECB but depends on all previously computed data blocks.

The cipher text block of the previous computation round is XORed to the current round's plain text block. While encrypting plain text blocks this XOR function takes place prior to the encryption function, for decrypting cipher blocks it takes place after the decryption function. The method is depicted in Figure 2.1 and can be formulated mathematically as follows:

Encryption: $\quad C_{i}=E_{K}\left(P_{i} \oplus C_{i-1}\right)$ Decryption: $\quad P_{i}=C_{i-1} \oplus D_{K}\left(C_{i}\right)$ 
$C_{i}$ and $P_{i}$ denote the cipher and plain text blocks, $E_{K}$ and $D_{K}$ represent the encrypt and decrypt functions.

By feeding back the previously generated cipher text, CBC circumvents the major problem of ECB which transforms plain text blocks of same content into cipher text blocks (result blocks) of same content. Using CBC, messages of same content will be processed into result messages of same content. This can be circumvented by initializating CBC using appropriate initialization vectors.

Unlike ECB, CBC mode shows error propagation since in case of a received cipher block containing an error not only is this block affected but also the following one before it recovers; much like ECB, however, it will not recover from synchronization errors [104].

There are certain security issues with CBC mode allowing attacks based on cryptanalysis which can be circumvented by slightly modifying the feedback pattern. Since security discussions are beyond the scope of this document, the interested reader is referred to [104].
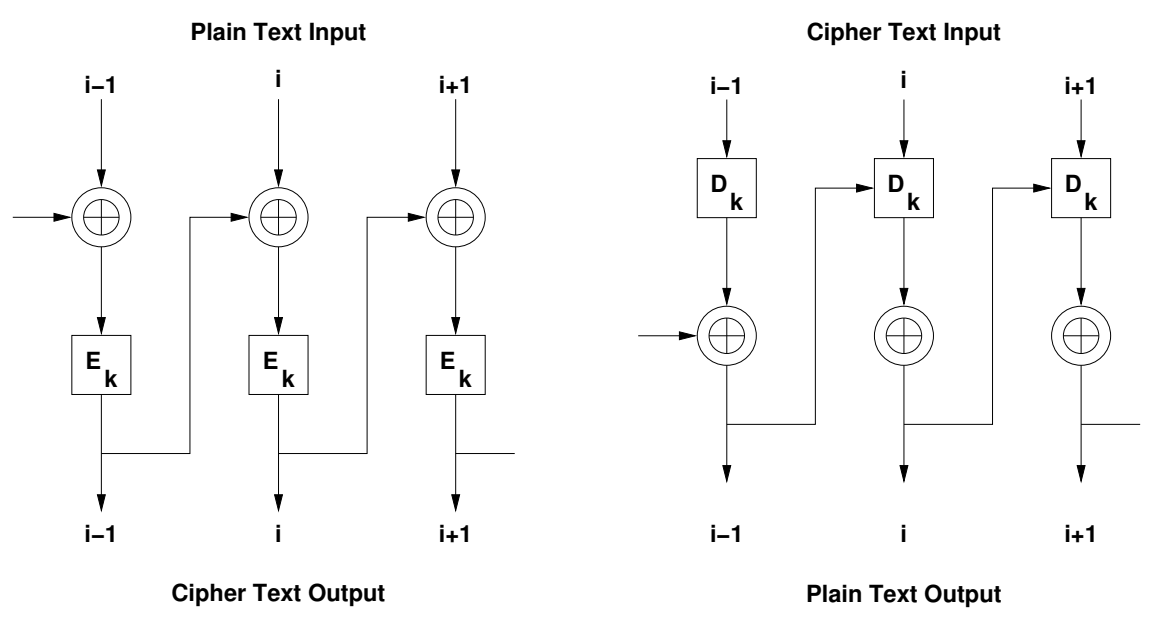

Figure 2.1: Encryption and Decryption using CBC 


\subsubsection{Cipher Feedback Mode (CFB)}

One of the problems with ECB and CBC modes is that they operate on block size rather than single characters or bits as it would be necessary for certain applications such as securing a data stream from or to a terminal: In this example an immediate reaction to single characters is necessary rather than collecting a complete block of characters prior to processing. The straightforward idea of padding a block - even with cryptographically sensible values - is not resonable since a potential attacker would know that the data payload of a complete block is only a single character. Instead of simple padding, a scheme as illustrated in Figure 2.2 is used.

The idea behind this scheme is easy to understand: Since a block algorithm can only work on entire blocks rather than symbols of arbitrary size where simple padding to block boundaries obviously is not reasonable a shift register is used to generate blocks of required length. This register is initialized by a cryptographically sensible value, the so-called initialization vector. Assuming a byte-wise encryption or decryption based on a 64-bit algorithm this shift register contains 8 positions. Whenever a character is sent out, the register content will be (de)scrambled using the (de)cipher key; the leftmost byte of the result of this operation is then XORed with the input byte and the register is shifted by one position to the left.

In case of encryption, the plain text byte is fed into the XOR operation and the resulting cipher byte is stored at the righmost position of the shift register. For decryption, the incoming cipher byte is stored there and is fed into the XOR operation; the result then is the plain text byte. This equals

$$
\begin{array}{ll}
\text { Encryption: } & C_{i}=P_{i} \oplus E_{K}\left(C_{i-1}\right) \\
\text { Decryption: } & P_{i}=C_{i} \oplus D_{K}\left(C_{i-1}\right)
\end{array}
$$

and Operation starts with index $i=1$ and an initialization vector $c_{0}$ is used as a "seed" for the process.

Of course, the feedback mechanism makes CFB less resistant against bit errors and more susceptive to error propagation: A packet containing an error bit will not only affect the current but also the $n$ following rounds where $n$ is the size of the shift register; the maximum boundary for error propagation is $\frac{n}{m}+1$ with $n$ being block and $m$ being symbol size. For the aforementioned 9 byte example, the current plus eight following rounds will 
retain an error. However, it will recover from synchronization errors since the error will age out of the shift register after $\frac{n}{m}$ steps.
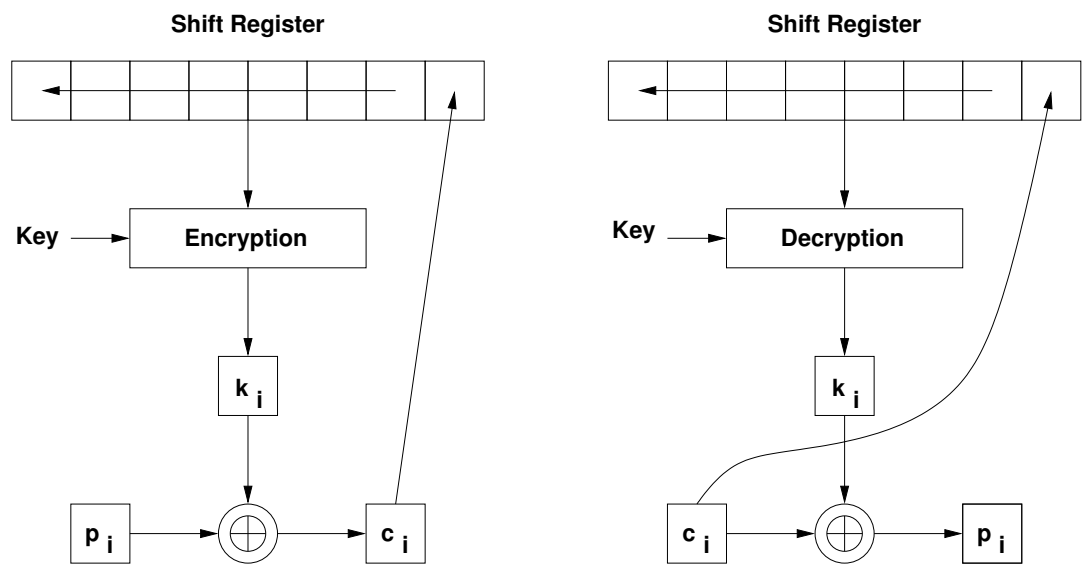

Figure 2.2: Cipher Feedback Mode (CFB)

\subsubsection{Output Feedback Mode (OFB)}

This mode is very similar to CFB. However, the symbol being fed back into the shift register is the cipher symbol $k_{i}$ generated from the shift register. Thus, the feedback mechanism and the contents of the shift register $S_{i}$ are completely independent from the cipher data stream but only depend on the cipher key [30] as illustrated by Figure 2.3. Hence, its formula can be written as:

$$
\begin{array}{lll}
\text { Encryption } & C_{i}=P_{i} \oplus S_{i} & S_{i}=E_{K}\left(S_{i-1}\right) \\
\text { Decryption } & P_{i}=C_{i} \oplus S_{i} & S_{i}=E_{K}\left(S_{i-1}\right)
\end{array}
$$

The advantage of $\mathrm{OFB}$ over $\mathrm{CFB}$ and $\mathrm{CBC}$ is that feedback computation can be done in advance and independently from the message if the size of message blocks sharing the same key is known in advance. Like in ECB mode this allows a more coarse grained parallelization since $k_{i}$ and $S_{i}$ are independent from the message itself.

Also, for the same reason errors in transmission do not propagate as in the previously discussed feedback modes. However, there are various 
security issues concerning the use OFB modes [68, 42, 43, 48] which the dedicated reader is referred to.

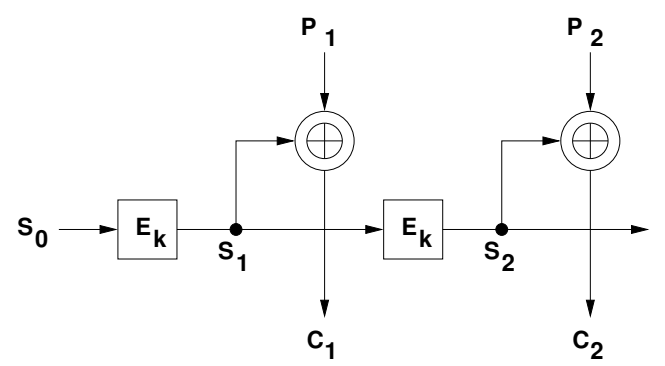

Figure 2.3: Output Feedback Mode (OFB)

\subsubsection{Counter Mode (CM)}

The security issues of OFB regarding block and feedback size (which have to be of same number for maximal security) as discussed in the previous section can be solved by using the Counter Mode (CM): Block ciphers using $\mathrm{CM}$ create their internal state from sequence numbers rather than output of the cipher algorithm itself $[44,51,69]$ where the counter is incremented by a constant value after each round. This mode has the same attributes as OFB concerning error propagation and loss of synchronization but allows the generation of output smaller than block size.

The nature of the used counter does not matter; it can be either a simple up-counter, be incremented by a certain offset, or constructed around pseudo-random number generators. Based on this counter, the cipher key, and a rather complicated output function, the cipher symbol $K_{i}$ is computed $[51,44]$ which will then be used for encryption or decryption of a message block.

\subsection{Summary}

Within this chapter a basic introduction into the field of cryptography was presented and definitions of the encryption and decryption process were given. Also, the difference between symmetric and asymmetric algorithms 
was worked out. The foci of this chapter are the various operation modes of crypto algorithms.

For security reasons, today mostly chained operating modes are used. For example, IPSEC defines each packet to have its own initialization vector which means that within a packet the blocks are "chained" together. As the name suggests, these modes include results of previous calculations. This already makes one basic decision about the processor architecture: Speeding up algorithm processing has to be achieved by single, powerful processor cores rather than a SIMD-like architecture with a certain number of processor cores working on different data chunks. Doing so would gain speed-up only for ECB mode. For chained modes a SIMD architecture would not necessarily lead to higher per-channel computation power, but provide multiple channels sharing the same crypto algorithm multiplying the overall throughput. Looking at the IPSEC example, such a SIMD architecture would allow multiple channels to be processed in parallel.

Similarly, also a MIMD approach would not provide higher per-channel throughput. Like a SIMD architecture it would offer multiple channels, but - contrary to SIMD - an individual crypto algorithm can be applied to each channel. 
2. CRypto Algorithms - Terms And Definitions 


\begin{tabular}{ll}
\hline \hline & $\mathcal{2}$ \\
\hline \hline
\end{tabular}

\section{Algorithm Discussion and Analysis}

\subsection{Exploitable Parallelism}

Network communication exposes many levels of possibly exploitable parallelism that can be used to improve throughput. This can happen on a perconnection base, for example by assigning one processing unit to one single connection. It also can happen on a per-packet base; each packet consists depending on the selected packet size for transmission and the used crypto algorithm - of several data blocks. With IPSEC in CBC mode, for example, each packet will use different initialization vector which means that packets can be processed individually.

Within one packet, the amount of exploitable parallelism is influenced by the operation mode used and the algorithm itself. Using ECB mode, each block of a packet can also be processed individually; this is not possible with feedback modes where the previously processed block is fed into the current block's encryption (or decryption) process. As mentioned in Section 2.2.1 ECB for security reasons is hardly used anymore which usually means that blocks cannot be processed individually because a data dependency exists between consecutive blocks of a packet.

On the block level, parallelism is dictated by data dependencies within the used algorithm and number of functional units. The following sections concentrate on selected cryptographic algorithms, possibly exploitable parallelism and additional requirements such as dedicated functional units. 


\subsection{Selection of Algorithms}

For this work an initial set of algorithms was chosen consisting of DES/3DES [26], AES/Rijndael [40], and MD5 [93].

DES was chosen since it is the very first standardized crypto algorithm. Despite its age it is still widely used, although software realization of DES is quite problematic since it is very hardware oriented containing many singlebit permutations rather than operations on complete data blocks. Additionally, it operates on a variety of data sizes which are 64-bit, 56-bit, 48-bit, 32-bit and 28-bit which are usually not ideally supported by standard architectures.

AES/Rijndael on the other hand is the new encryption standard and was especially designed to run on current general purpose architectures. Here, the challenge is developing an architecture which supports AES/Rijndael best in all configurations but also does not contain too many AES-specific elements.

The third candidate, MD5, is a widely used hash algorithm which was chosen to demonstrate the flexibility and universability of the architecture described in this work. It is very similar to other hash algorithms like its predecessor MD4 [92] or SHA-1 [27, 108] which are also included within this chapter.

Also, two further algorithms, IDEA [73, 101] and RC6 [96] were added; IDEA was developed in the beginning 1990s as an algorithm easily and efficiently implementable on 16-bit architectures and gained popularity through the PGP tool mainly used for securing email privacy. RC6 is a recent algorithm and was one of the finalists in the AES competition.

\subsection{The Data Encryption Standard (DES)}

DES is the first standardized crypto algorithm. It was developed by IBM in the 1970s and published in the Official Gazette of the United States Patent and Trademark Office in 1975 [126] and 1976 [127]. In 1977 DES became a Federal Information Processing Standard (FIPS) [26]. It fulfills the later defined Security Requirements for Cryptographic Modules [28] which specify security requirements for protecting classified information within computer and telecommunication systems. 
The DES algorithm can be used in four different modes [70] which are Electronic Codebook mode (ECB), Cipher Block Chaining mode (CBC), Cipher Feedback mode (CFB), and Output Feedback (OFB). A discussion of these operation modes and their architectural impact was already given in Section 2.2.

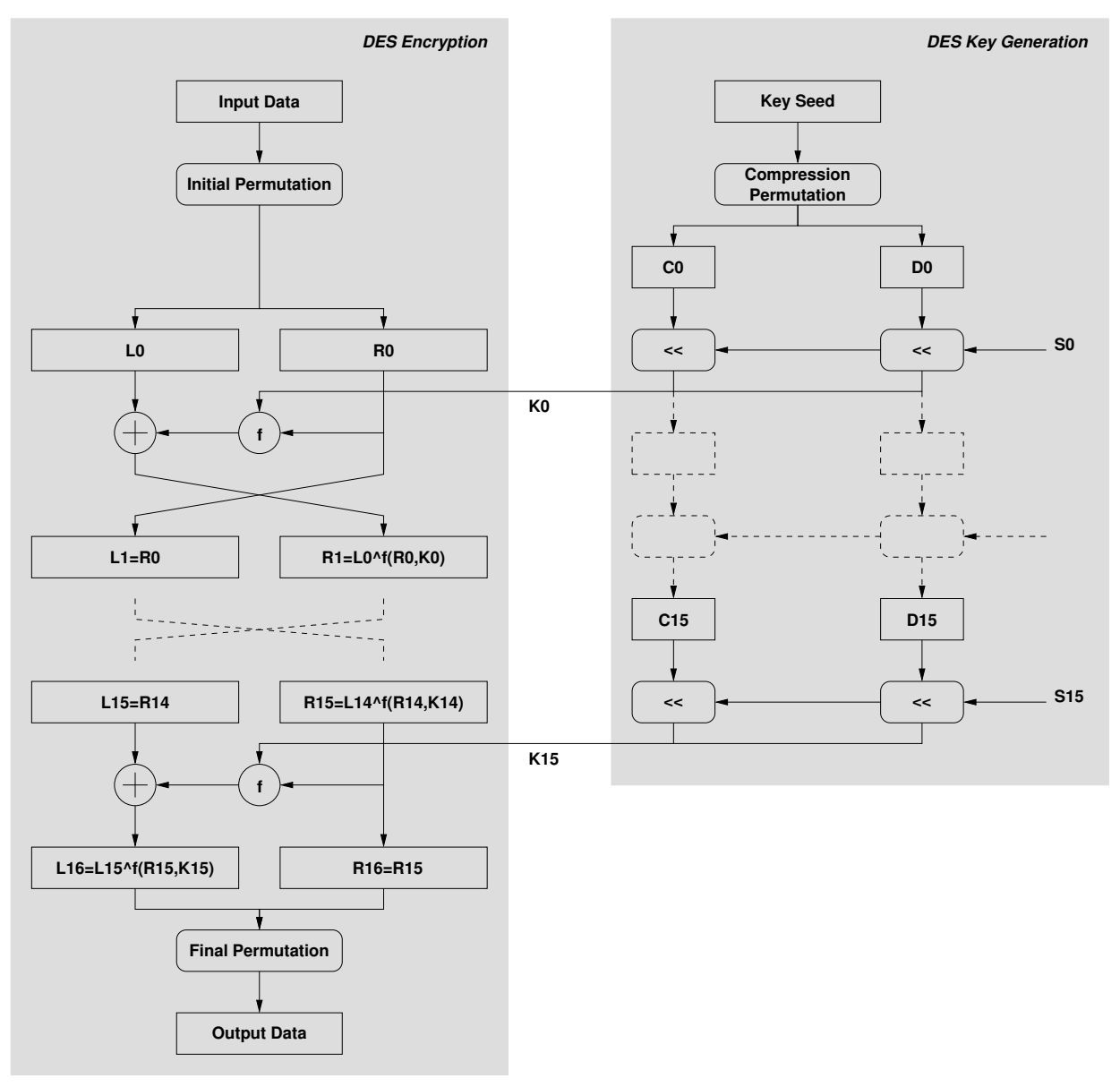

Figure 3.1: Workflow of the DES Algorithm 


\subsubsection{Design of the DES Algorithm}

The DES algorithm basically consists of three parts which are initial permutation, 16 rounds of encryption and final (inverse initial) permutation as shown in Figure 3.1. It operates on 64-bit chunks of data, hence any message which needs to be encrypted using DES has to be padded with appropriate filling data to the next 64-bit boundary. Also the key provided for en- or decryption needs to be 64-bit, however, every eighth bit is dropped during the key permutation resulting in an effective bit length of 56-bit. The remaining 8 bits can be used for parity checks but do not contribute to cryptographic strength.

Within each round, key generation and encryption take place. Key generation is fairly easy: The 56 bits of key data are split into halves; each half is fed into a 28-bit cyclic left-shift register which according to a round constant rotate the key halves up to two positions.

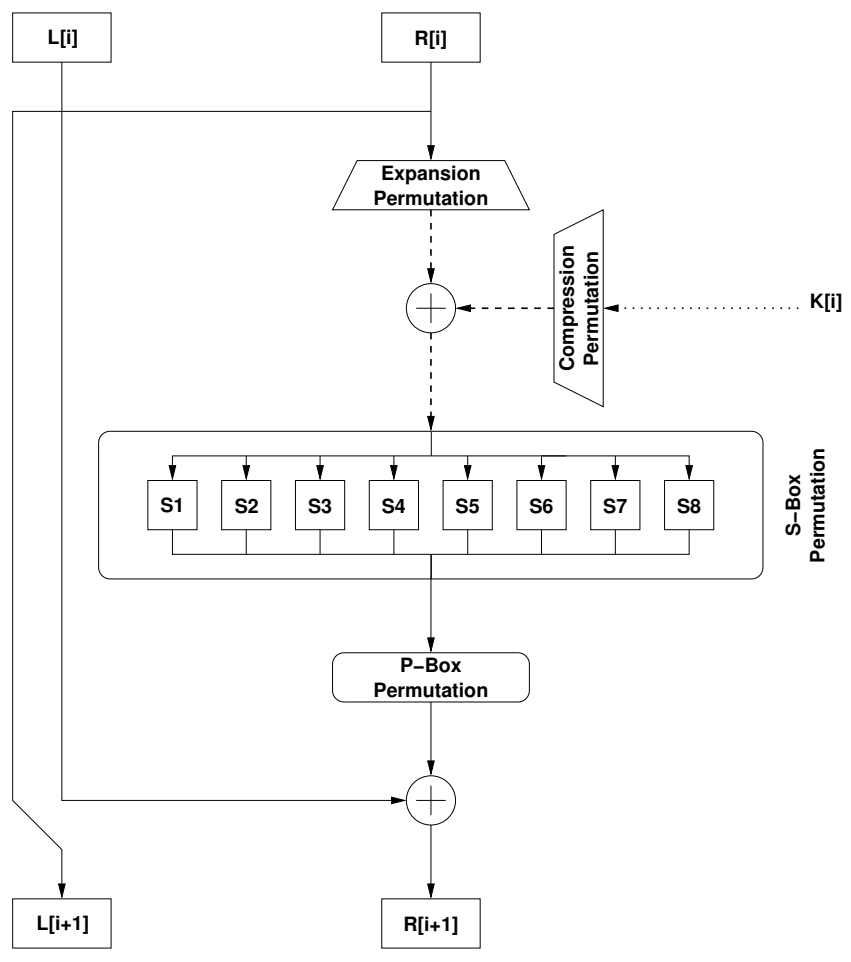

Figure 3.2: A single DES round 
The encryption process itself (see Figure 3.2) is slightly more complicated: The input data is split into two chunks of 32 bits each where only the right half is included into the encryption function. Through an expansion permutation these right 32 bits are expanded to 48 bits; similarly, the 56-bit key is compressed to 48 bits. Both values are then XORed, the result of this operation is fed into a lookup table implemented as $8 \mathrm{~S}$-Boxes. These S-Boxes provide the main encryption (or decryption) function within the DES algorithm. All other permutations employed are used to evenly scatter the input data among a 64-bit chunk ensuring that no conclusions about message and key can be drawn by analyzing multiple chunks of encrypted data.

Functionally, each S-Box is a ROM with 32 memory locations: It translates a 5-bit input value into a corresponding unique 4-bit output value. No two S-Boxes are the same. The 48-bit result of the previously performed XOR operation between compressed round key and expanded right half of the input data is fed into the array of S-Boxes producing a 32-bit lookup value. This value undergoes another permutation, the P-Box permutation, before it is XORed with the left half of the input data.

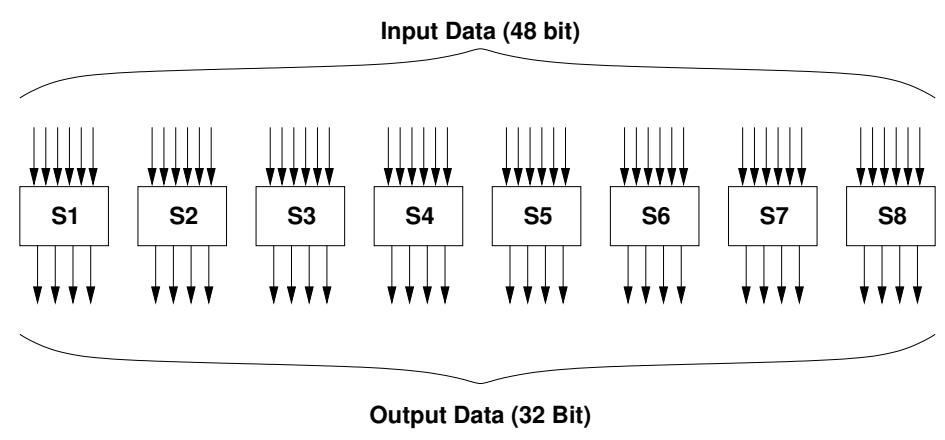

Figure 3.3: The DES S-Box

For the next round, the computed result becomes the left half of the input data where the previous left half is swapped with the right half. This swapping is omitted in round 16, instead the 32-bit values are concatenated and sent through the final permutation which is the reverse operation of the initial permutation.

Since DES is a symmetric algorithm decryption works just the same as encryption, only the round keys have to be applied in reverse order. 


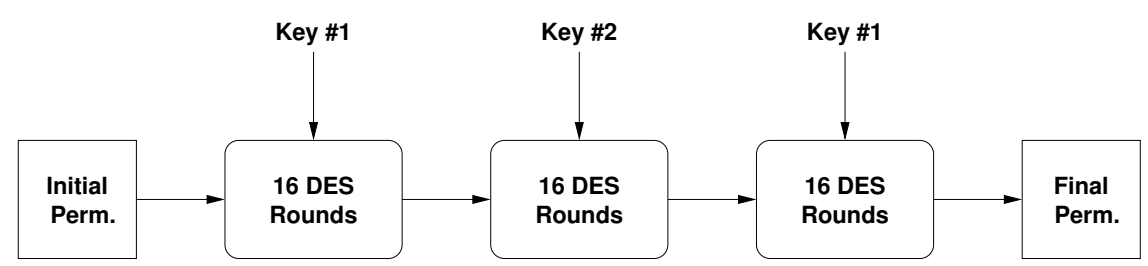

Figure 3.4: Triple-DES

Today, plain DES usually is not used anymore for security reasons since with modern machines it can be broken in comfortable time using bruteforce methods (for example, the DES-II challenge was won on July 15, 1998 using the DES Key Search Machine after 56 hours [1]; half a year later, the DES-III challenge was won on January 18, 1999 after less than 24 hours [2]). Instead, Triple-DES - usually referred to as 3DES - is used: This means, that each data packet is DES encrypted three times using two different keys as shown in Figure 3.4.

\subsubsection{Analysis of the DES Algorithm}

Figure 3.1 shows very clearly that the DES algorithm divides into three steps which are input permutation together with key transformation, encryption, and final permutation. The main time consuming factor of DES is the encryption since it is performed 16 times (48 times with 3DES).

\subsubsection{Speeding up Encryption}

Recalling Figure3.2 displays the encryption process consisting of five major steps; assuming these can be computed in 5 cycles this leads to 80 cycles in total for a complete DES encryption, 240 cycles for 3DES which are itemized in Table 3.1.

It is obvious that each permutation is followed by an accompaning XOR operation. This XOR/permutation compound takes two inputs, one of which is permutated prior to the XOR operation, the other is fed in directly. With this observation DES encryption can be reformulated as follows needing only 48 (144 for 3DES) cycles as dissected in Table 3.2. 
1. Expansion-Permutation of $R_{i-1}$, transfer $R_{i-1}$ to $L_{i}$

2. XORing the result from step 1 with the generated round key

3. Perform an S-Box lookup based on the XOR result

4. Do a P-Box Permutation on the lookup value

5. XOR that result with the value of $L_{i-1}$ and store the result into $R_{i}$

Table 3.1: Naive DES Implementation

1. Expand/XOR $R_{i-1}$ with the actual round key, transfer $R_{i-1}$ to $L_{i}$

2. Perform an S-Box lookup based on the Expand/XOR result

3. P-Box permutate/XOR the lookup value with $L_{i-1}$ and store the result into $R_{i}$

Table 3.2: A faster DES Implementation

1. Initial expansion/XOR function followed by an S-Box lookup ( 2 cycles)

2. 14 iterations of the monolithic function followed by an SBox lookup ( 2 cycles, 28 cycles in total)

3. A final P-Box/XOR function (1 cycle)

Table 3.3: An even faster DES implementation (excluding key generation)

1. One cycle from the initial permutation and key transformation

2. 31 (3DES: 93) from the encryption (key switch within 3DES can be happen in parallel to the P-Box/XOR function)

3. One cycle from the final permutation

Table 3.4: Ideal DES implementation including Round Key generation 
Since 16 consecutive rounds of encryption (or decryption, which is basically the same algorithm) occur, P-Box/XOR and Expansion/XOR happen sequentially for the inner fourteen rounds and can be further condensed to a monolithic three-input function which takes the result of an S-box lookup, $L_{i-1}$ and the round key and produces the index value for the S-box lookup. Within this function also the transfer from $R_{i-1}$ to $L_{i}$ also takes place.

Using such a function, the 16 rounds of encryption can be further shrunk bringing the complete DES encryption down to 31 (93 for 3DES) cycles as explained in Table 3.3 leading to an ideal (3)DES implementation listed in Table 3.4.

\subsubsection{Round Key Calculation}

Round key calculation consists of two steps which are key shifting and key compression. Since this computation is not dependent on any other input data besides the shift value and the content of the key register this process can happen in parallel to the encryption. Ideally, round key calculation is a combined operation like expansion/XOR or P-Box/XOR and takes only one cycle.

On an ideal parallel architecture calculation of the round key can take place during an S-Box lookup for rounds 2 to 16; the first round's key calculation can happen in parallel to initial permutation needing key transformation prior to rotation. In that case the round key generation can be completely overlapped with the main encryption algorithm without incurring extra cycles.

\subsubsection{Proposed Computation Speed}

Following the above assumptions based on an ideal architecture, the encryption of a complete 64-bit data block takes 33 (3DES: 95) cycles resulting in 0.52 (1.48) cycles per bit or a throughput of 775 (3DES: 270) MBit/s assuming that the architecture is running at $400 \mathrm{MHz}$ and offers capabilities as listed in Table 3.5. 


\begin{tabular}{|c|c|}
\hline Parallelism & $\begin{array}{l}\text { - Arithmetic operations can happen in parallel } \\
\text { with memory lookup (round key generation to- } \\
\text { gether with S-Box lookup) } \\
\text { - Two arithmetic operations can be processed in } \\
\text { parallel (round key generation together with in- } \\
\text { put permutation) }\end{array}$ \\
\hline Instruction Set & $\begin{array}{l}\text { - DES Input Permutation } \\
\text { - DES Final Permutation } \\
\text { - combined DES expansion/XOR operation } \\
\text { ation } \\
\text { - method to combine the above two operations } \\
\text { into one monolithic operation } \\
\text { - monolithic round key generation operation con- } \\
\text { sisting of switchable key transformation, key } \\
\text { rotation, and key compression }\end{array}$ \\
\hline Data Size & $\begin{array}{l}\text { - Input/Output: } 64 \text { bit } \\
\text { - Intermediate Results: } 28,32,48 \text {, and } 56 \text { bit }\end{array}$ \\
\hline
\end{tabular}

Table 3.5: Architectural requirements for efficient (3)DES implementation 


\subsection{Advanced Encryption Standard (AES / Rijndael)}

When this work was started the Rijndael algorithm [39] was one of the participiants in the contest for becoming the new encryption standard. At the end of 2001 it won against its competitors and became the so-called Advanced Encryption Standard (AES) [40] which over time will replace DES and possibly many other, not officially standardized crypto algorithms, at least in the commercial and governmental area.

Unlike DES which was designed to easily fit into 1970s hardware technology AES/Rijndael was developed to efficiently run in software on modern general purpose processors. Where DES is very easy to implement in hardware it tends to be quite slow in software due to exhaustive bit shuffling and internal changes in data chunk sizes (56-bit to 48-bit, 48-bit to 32-bit, 4-bit to 6-bit, etc.); in AES/Rijndael data chunks are either 8-bit or 32-bit and all internal operations work on these chunk sizes. For block and key size usually multiples of 32 bits are given; in this context, a size of 4 means $4 * 32=128$ bits.

A further advantage of the AES/Rijndael algorithm over DES is its flexibility to operate on different key and data block sizes ${ }^{1}$. Encryption strength can be adjusted to the actual needs as shown in Table 3.6.

\begin{tabular}{|c|c|c|c|}
\hline & \multicolumn{3}{|c|}{ Block Size } \\
\hline & 4 & 6 & 8 \\
\hline 4 & 10 & 12 & 14 \\
\hline 6 & 12 & 12 & 14 \\
\hline 18 & 14 & 14 & 14 \\
\hline
\end{tabular}

Table 3.6: Number of Rounds as a function of block and key size

The AES/Rijndael algorithm as realized in the reference implementation can be divided into three phases: Round key generation and initialization, first round and remaining rounds. For decryption, the latter two phases become "first rounds" and "last round". The phases are illustrated in Figures

\footnotetext{
${ }^{1}$ It must be noted, however, that usually the data block size is 128 bits. This means, that only three of nine possible configurations are used.
} 


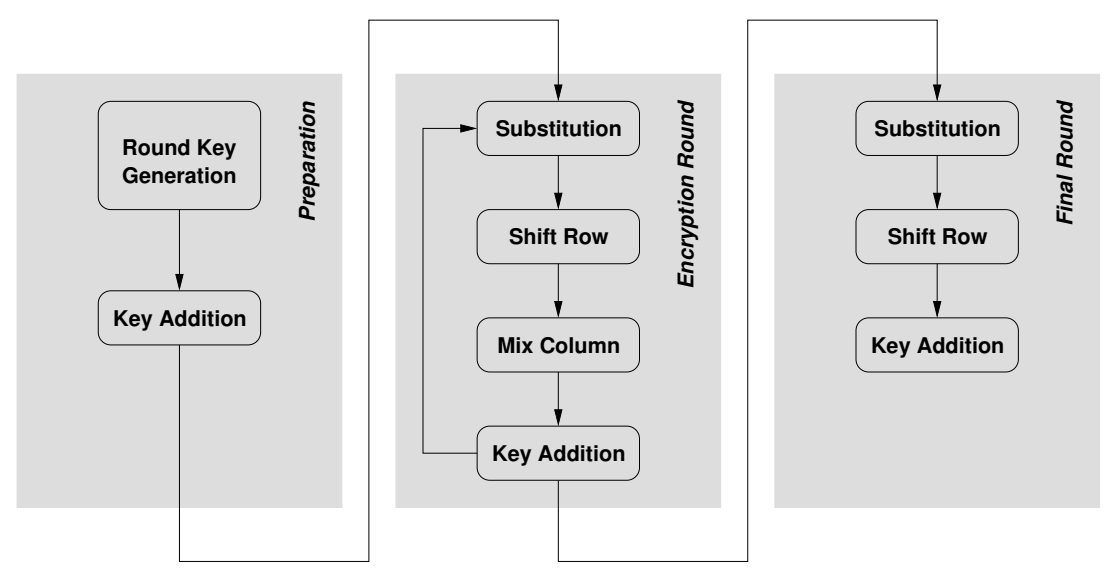

Figure 3.5: AES Encryption

3.5 and 3.6. The rounds themselves split into round key addition, substitution, row shifting, and column mixing or their inverse operation for decryption. Again, the main cryptographic operation is a non-linear transformation based on an S-Box lookup which happens during the substitution stage.

\subsubsection{Key Scheduling}

AES/Rijndael does not directly operate on the cipher key or a primitive permutation of it. Instead, a sequence of round keys is generated according to the following rules:

- The number of round key bits must equal the block length times the number of rounds plus one. Assuming a block length of 128 bits and a key of same size this results in 10 rounds or $128 \cdot(10+1)=1408$ needed round key bits.

- For this purpose the cipher key is expanded into an expanded key. From this expanded key the round keys are taken in blocks of $N_{b}$ words where $N_{b}$ denotes the block size in bits divided by 32 .

The expansion is done recursively using rotation and exclusive-or operations where the expansion algorithm is dependent from the key size $N_{k}$ which is the key size in bits divided by 32. This algorithm is explained 


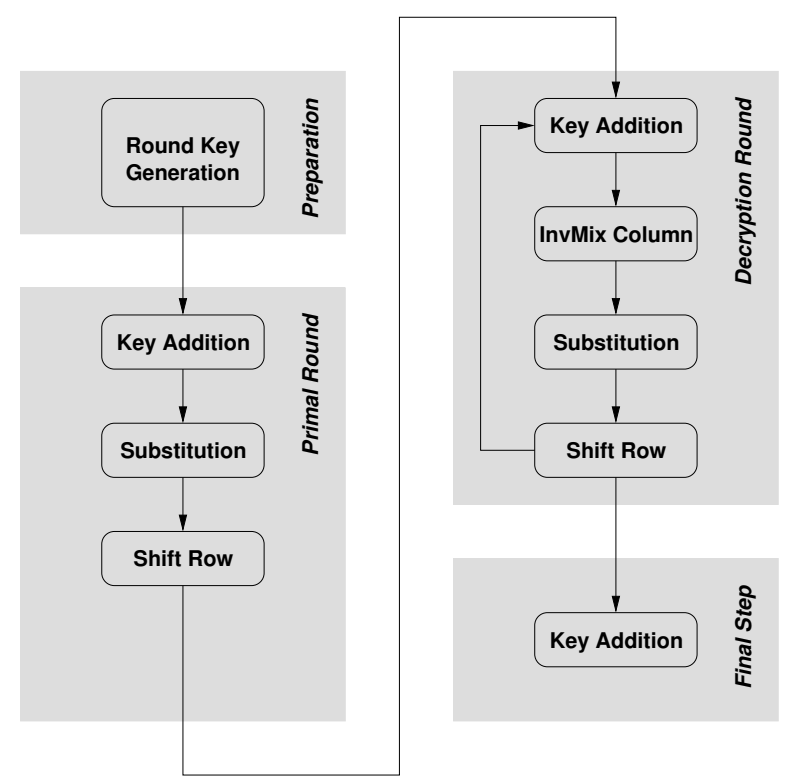

Figure 3.6: AES Decryption

on pages 14 to 15 of [39] and generates the expanded key array $W[i]$ from which round keys of desired block size are taken.

Although the reference implementation - for the sake of simplicity and intuition - precomputes all round keys, the algorithm is designed in a way that round key generation can be done on the fly and interwoven with encryption or decryption. This was done in a fast AES-128/128 implementation [45].

\subsubsection{Inner architecture of AES/Rijndael}

As explained before, AES/Rijndael encryption or decryption divides into four different steps which are explained below. For the following explanations the nomenclature of the Rijndael description using the term state applies [40, 39]:

The different transformations operate on the intermediate result, called the state. This state can be pictured as a rectangular 
array of bytes with four rows and a variable number of columns equal to the block length divided by 32 .

So whenever the term State is used in the following explanation the intermediate result of an ongoing encryption or decryption is meant.

\section{- Key Addition}

During this operation a round key $k$ is applied to the State $a$ by a bitwise exclusive-or operation. The round keys must have been computed from the cipher key by the key scheduler prior to addition and equals the block length in length. Mathematically expressed, key addition performs the following operation:

$$
\forall i, j: b_{i, j}=a_{i, j} \oplus b_{i, j}
$$

\section{- Substitution}

This operation replaces each element of the State $a$ by its referring entry in the S-Box table and is performed by a simple table-lookup and write-back algorithm:

$$
\forall i, j: a_{i, j}=\operatorname{sbox}\left[a_{i, j}\right]
$$

Unlike DES where the entries of the S-Box tables were designed by the NSA giving no further insight into the construction of those tables the creation of the AES/Rijndael S-Box is well documented and can be found in Chapter 4.2.1 of [39].

\section{- Row Shifting}

In this stage, rows 1 to 3 of the State are cyclically shifted over different offsets where the shift offsets are individual per row and are dependend on the block length $N_{b}$. Row 0 is not shifted. Table 3.7 shows the shift offsets depending on block lengths.

\begin{tabular}{|c||ccc|}
\hline$N_{b}$ & Row 1 & Row 2 & Row 3 \\
\hline \hline 4 & 1 & 2 & 3 \\
6 & 1 & 2 & 3 \\
8 & 1 & 3 & 4 \\
\hline
\end{tabular}

Table 3.7: Shift offsets for different block lengths 


\section{- Column Mixing}

In this stage, the columns of the State are considered as polynomials over $\operatorname{GF}\left(2^{8}\right)$. They are multiplied modulo $x^{4}+1$ with a fixed polynomial $c(x)$ given by

$$
c(x)={ }^{\circ} 033^{\prime} x^{3}+{ }^{\prime} 01{ }^{\prime} x^{2}+{ }^{\prime} 01{ }^{\prime} x+{ }^{\prime} 02{ }^{\prime}
$$

Because this polynomial is coprime to $x^{4}+1$ and therefore invertible, it can be written as a matrix multiplication following the formula

$$
\left(\begin{array}{l}
b_{0} \\
b_{1} \\
b_{2} \\
b_{3}
\end{array}\right)=\left(\begin{array}{llll}
02 & 03 & 01 & 01 \\
01 & 02 & 03 & 01 \\
01 & 01 & 02 & 03 \\
03 & 01 & 01 & 02
\end{array}\right) \cdot\left(\begin{array}{l}
a_{0} \\
a_{1} \\
a_{2} \\
a_{3}
\end{array}\right)
$$

\subsubsection{Implementational aspects of AES/Rijndael}

For the other algorithms discussed in this chapter due to their simplicity there is hardly a difference between optimized and reference implementation especially when considering hardware implementation. AES/Rijndael is different since it has been designed to run especially well in software. The algorithm itself is well described both in the NIST standard paper and the reference implementation, for evaluating its architectural demands it must be assumed that there is a noticeable difference between an evaluation based on the reference implementation and an optimized version. For this reason, the reference implementation of this algorithm has been reverse-engineered to get the "naked" (not optimized for software implementation) algorithm.

This work is based on an algorithm analysis and evaluation documented in [45]. The implementation based on this analysis is able to perform AES$128 / 128$ encryption within 80 cycles including round key generation as opposed to 60 cycles excluding round key generation for the straight-forward implementation documented in [46].

\subsubsection{Architectural Impact}

[46] already demands supporting operations which are further extended by [45]. Unlike DES, the needed operations are not explicitly AES-specific and can be used for other algorithms as well. 
AES/Rijndael was created with 32-bit architectures in mind. All operations within the algorithm operate on 32-bit (for computation) or 8-bit (for table lookups) quantities, the latter can be avoided by aligning the 8-bit table contents to 32-bit borders. Unlike with RC6, which will be discussed later in this chapter, this behaviour remains consistant throughout all AES configurations. Data and key size do not influence operand sizes, only computation effort. On the other hand, with RC6 the computation effort stays constant and the operand sizes vary. Unlike (3)DES, intermediate results always have 32 bits.

Since DES favours a 64-bit architecture, [46] and [45] were developed having a hypothetical 64-bit architecture in mind which led to the requirements as listed in Table 3.8.

\begin{tabular}{|ll|}
\hline Parallelism & - Two parallel arithmetic operations per cycle \\
& sufficient for encryption \\
Instruction Set & - S-Box lookup \\
- Swap-Operation for swapping 32-bit halves of & 64-bit registers \\
- Splitted 32-bit shift/rotate allowing two 32-bit & halves of one register to be shifted by two indi- \\
& vidual amounts \\
- & Fold-Operations (see 5.7.3.1) \\
- Bit-"Butterfly"-Operations & \\
& - 32 -bit for arithmetic operations \\
Data Size & 8-bit for table lookups (see text) \\
\hline
\end{tabular}

Table 3.8: Architectural requirements for efficient AES/Rijndael implementation 


\subsection{The MD5 Message Digest Algorithm}

The MD5 message digest algorithm [93, 107] generates a 128-bit hash value from an input message. Due to the algorithm's workflow the message size has to be a multiple of 512 bits (minus 64 bits) which is achieved by padding the message with a single one bit followed by null bits until the next "512 bits minus 64"-boundary is reached. The final 64-bit field is then filled with a representation of the original message length. This padding mechanism is illustrated in Figure 3.7. The overall length divided by 512 determines the number of MD5 iterations where each iteration processes one 512-bit block of the padded message.

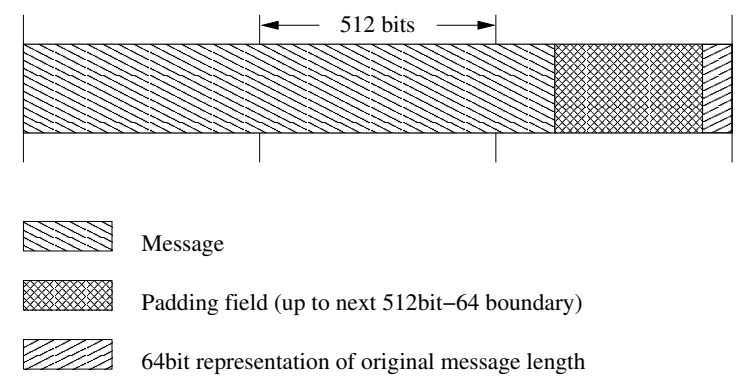

Figure 3.7: Message Padding for MD5

$$
\begin{aligned}
& A=0 x 01234567 \\
& B=0 x 89 a b c d e f \\
& C=0 x f \text { edcba98 } \\
& D=0 x 76543210
\end{aligned}
$$

Table 3.9: Chaining Variables Init Values

The hash value is based on four so-called chaining variables, each being 32-bit wide. They are named $A$ to $D$ and get initialized with the values shown in Table 3.9.

An MD5 iteration as illustrated in Figure 3.8 is based on four rounds of identical architecture. The current 512-bit block is split up into four 128-bit chunks each being fed into one round which consists of 16 steps. Within each round step as shown in Figure 3.9 the values of B, C, and D are fed into 
a non-linear function. The result of this function is XORed with a 32-bit excerpt of the j-th 128-bit chunk $M_{j}$ of the message block being currently processed, and the resulting value is then XORed with a 32-bit value taken from a lookup table $t_{i}{ }^{2}$. This first intermediate result is then circularly shifted left by $s$ bits where $s$ is one of four values taken from the actual round table. Finally, the computed value is XORed with $B$, the chaining variables are rotated clockwise so that $\mathrm{B}$ becomes $\mathrm{A}, \mathrm{C}$ becomes $\mathrm{B}, \mathrm{D}$ becomes $\mathrm{C}$ and into $A$ the result of the aforementioned computation is stored.

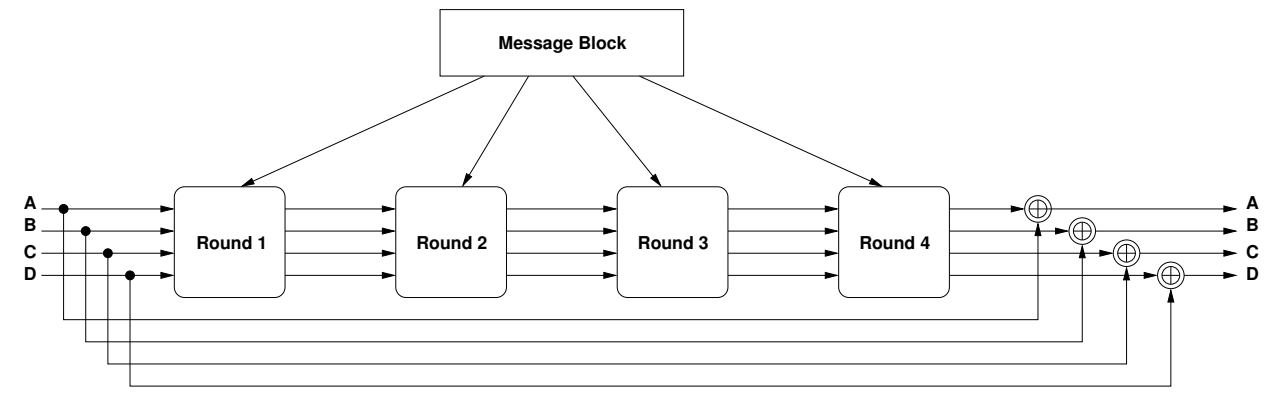

Figure 3.8: Workflow of the MD5 Algorithm

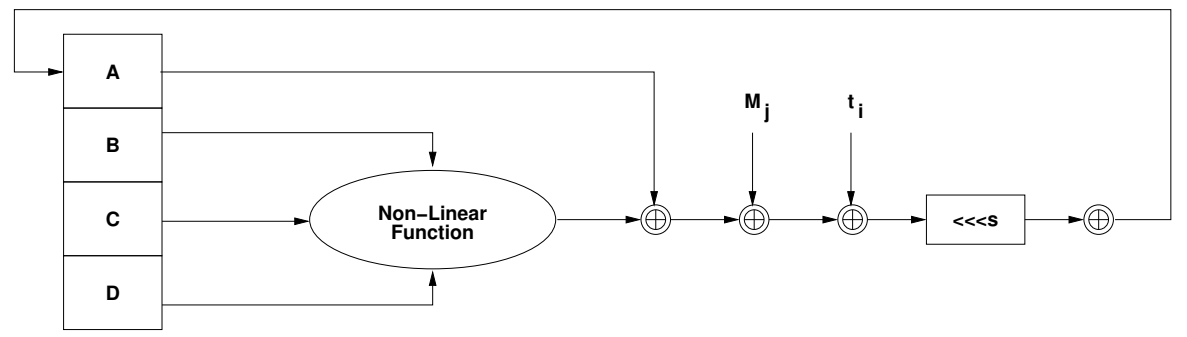

Figure 3.9: A single MD5 round

The hearts of the MD5 hash generation are non-linear functions employed in each round and round step. Each round uses an individual function which is listed in Table 3.10. These functions are designed in a way that the result's single bits are independent from each other and evenly distributed (assuming that this is also valid for the input values $X, Y$, and $Z$ ).

\footnotetext{
${ }^{2}$ The values of this table are based on the formula $2^{32} * a b s(\sin (i))$ [107].
} 
Having processed all 512-bit message chunks the resulting MD5 hash is the concatenation of the four chaining variables.

\begin{tabular}{|c|c|}
\hline Round 1: $\quad f(X, Y, Z)$ & $=(X \wedge Y) \vee((\neg X) \wedge Z)$ \\
\hline Round 2: $\quad g(X, Y, Z)$ & $(X \wedge Y) \vee(Y \wedge(\neg Z))$ \\
\hline$h(X, Y, Z)$ & $X \oplus Y \oplus Z$ \\
\hline$i(X, Y, Z)$ & $Y \oplus(X \vee(\neg Z))$ \\
\hline
\end{tabular}

Table 3.10: The non-linear functions

\subsubsection{Analysis of the MD5 Algorithm}

As shown in Figure 3.8 MD5 consists of four different rounds with 16 iterations each. Since there is not much outside the round iterations besides chaining variable initialization and final addition, the main computation time is used within the four rounds which therefore determine computation speed.

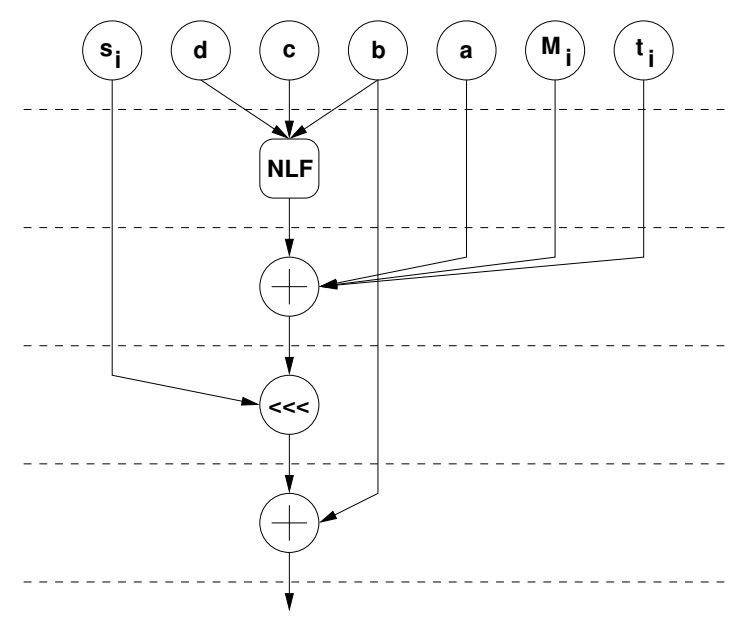

Figure 3.10: MD5 Data Dependencies

\subsubsection{Round Analysis}

A generic round has a structure as depicted in Figure 3.10 and initially consists of two parallel paths which are the computation of the non-linear func- 
tion (NLF) plus the addition of $a, M_{j}$, and $t_{i}$. The results of these operations are then added and shifted, finally $b$ is added to this result. The final sum is stored in the chaining variable $a$, then the registers are rotated and the next round begins. After the final step instead of the register rotation the initial values of the chaining variables are added onto their computed values.

\subsubsection{The non-linear functions}

Looking at the non-linear functions as sketched in Figure 3.11 shows an almost identical data flow for these: One parameter is negated, combined with another parameter and this result is in term combined with the third parameter.
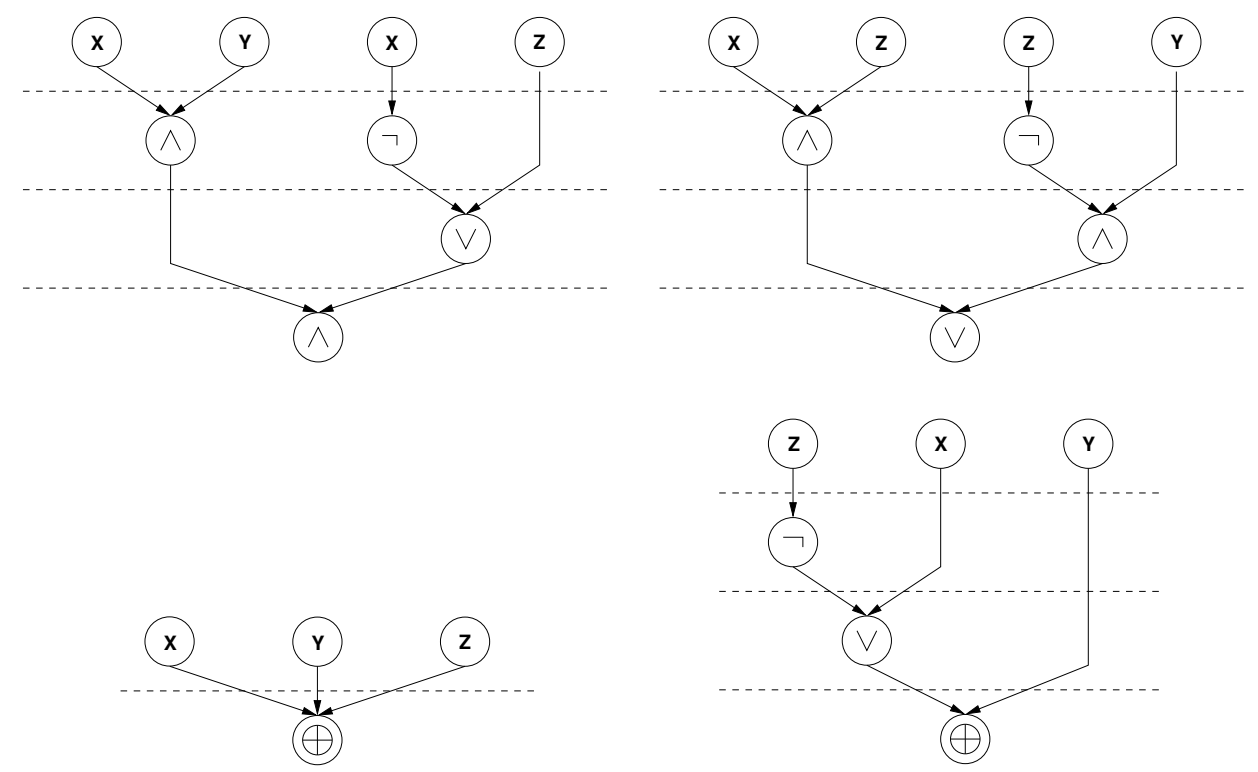

Figure 3.11: The MD5 non-linear functions F, G, H, and I

\subsubsection{Similar Hash Algorithms}

There are some hash algorithms which are closely related to MD5 and similarly wide-spread in use: These are namely the MD4 Message Digest algorithm [92, 91], and the Secure Hash Algorithm (SHA) [27, 108]. The latter 
is superficially similar to MD4 and MD5. However, a closer look reveals that SHA is quite different regarding parallelism which will be discussed in 3.6.

$$
\begin{array}{ll}
\text { Round 1: } & f(X, Y, Z)=(X \wedge Y) \vee(\neg X) \vee Z \\
\text { Round 2: } & g(X, Y, Z)=(X \wedge Y) \vee(X \wedge Z) \vee(Y \wedge Z) \\
\text { Round 3: } & h(X, Y, Z)=X \oplus Y \oplus Z
\end{array}
$$

Table 3.11: MD4 non-linear functions

Since MD4 is the direct predecessor of MD5 it is not surprising that it is a less complex version of MD5. It consists of only three functions and also misses the final addition of the chaining variable $b$ to the result of the shifting operation. Besides, MD4 uses slightly different non-linear functions as listed in Table 3.11. It also uses a different message block indexing for $M_{j}$ and a different look-up table $t_{i}$.

For a discussion of computation speed and architectural impact of MD5 see Section 3.6.1 which will also cover SHA.

\subsection{The Secure Hash Algorith (SHA)}

SHA [27, 108] was designed for use with the Digital Signature Standard [41] and is also very similar to MD5, but unlike that algorithm it employs five 32-bit chaining variables (producing a 160-bit hash) instead of four (resulting in a 128-bit hash) and 20 iterations per round instead of 16. Also, it applies rotate functions to those chaining variables not employed in the current non-linear function. However, where it is slightly more complex on the computational side as it only uses four round constants, one for each round. MD5, in contrast, uses individual constants for each iteration. SHA uses the same three non-linear functions as MD4 (see Table 3.11 where $g(X, Y, Z)$ is applied to rounds 2 and 4); their data flow is shown in Figure3.13.

However, as Figure 3.12 reveals, there are lesser data dependencies within an SHA round. Where MD5 has 3 sequential steps following the computation of the non-linear function, only one exists with SHA. This means that based on the data dependency graph the non-linear function (NLF) is the main time consuming factor within SHA with 50 to 75 per- 
cent, where with MD5 the NLF's contribution to the overall computation is between 25 to 50 percent.

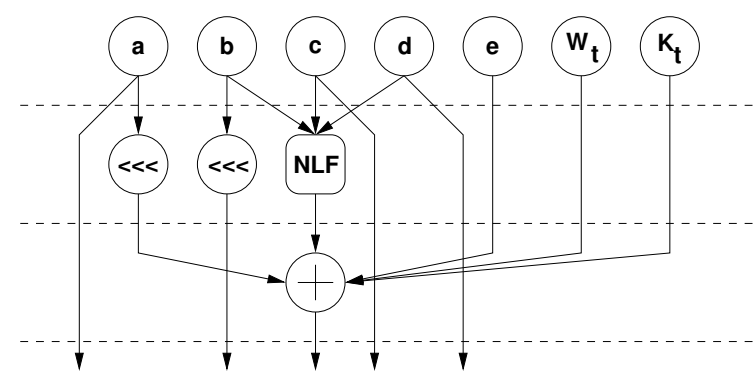

Figure 3.12: SHA Data Dependencies

\begin{tabular}{|rll|}
\hline Round 1: & $f(X, Y, Z)$ & $=(X \wedge Y) \vee(\neg X) \vee Z$ \\
Rounds 2 and 4: & $g(X, Y, Z)$ & $=X \oplus Y \oplus Z$ \\
Round 3: & $g(X, Y, Z)$ & $=(X \wedge Y) \vee(X \wedge Z) \vee(Y \wedge Z)$ \\
\hline
\end{tabular}

Table 3.12: The SHA non-linear functions

\subsubsection{Proposed Computation Speed and Architectural Im- pact}

The time determining factors within SHA are the non-linear functions; on an ideal architecture these vary between 1 and 3 cycles which means that this architecture needs to perform both shifts in parallel to the NLF computation resulting in 3 parallel ALUs. It will also need 3 -input logical operations $(\wedge$ and $\oplus$ ) and a 5-input addition; when limiting to 3-input logical operation a fourth ALU would be required since the 5-input addition needs to be split into two 3-input additions.

Assuming an ideal architecture we get the following numbers for all 4 rounds per 20 iterations: $1+20 *(4+2+3+2)+1=222$ cycles per 512-bit chunk equalling 0.43 cycles/bit or 2.31 bits/cycle for an ideal SHA processor as opposed to $1+16 *(6+6+4+6)+1=354$ cycles per 512-bit chunk which equals 0.69 cycles/bit or 1.44 bits/cycle for an ideal 

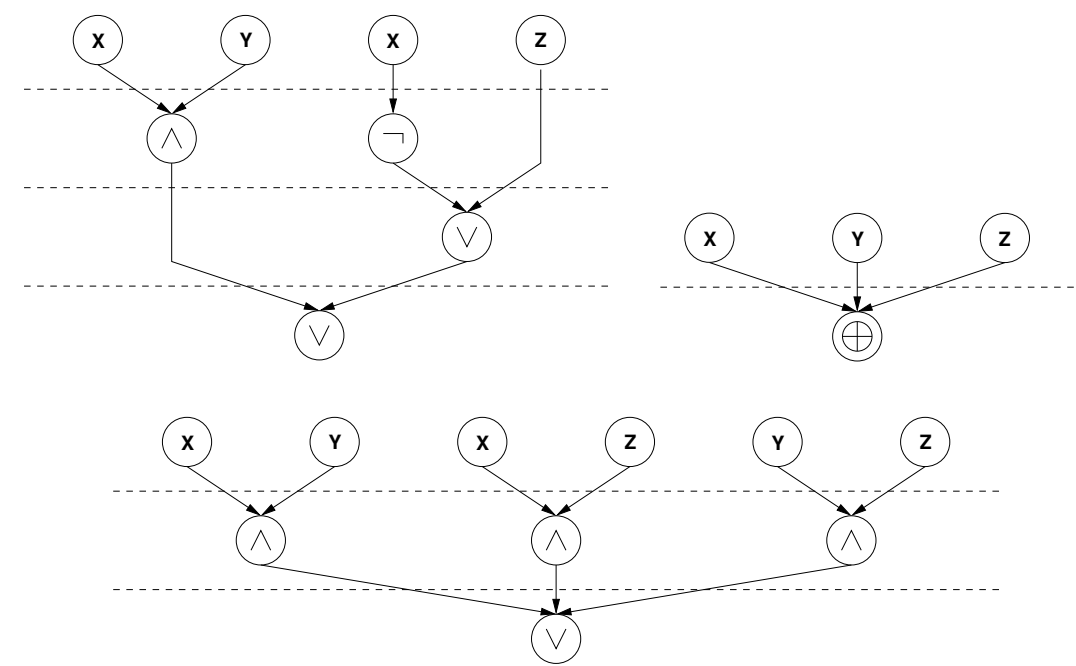

Figure 3.13: The SHA non-linear functions for rounds 1, 2/4, and 3

\begin{tabular}{|ll|}
\hline Parallelism & - up to 5 parallel arithmetic operations \\
& - up to 4 input variables needed per computation \\
& step \\
Instruction Set & - special monolithic functions not necessary \\
& - need for indexed addressing to access round ta- \\
& bles \\
Data Size & Input: 512 bit arranged in 32-bit chunks \\
& Output: $128 / 160$ bit (MD5/SHA) arranged in \\
& $32-$ bit chunks \\
& Intermediate Results: 32 bit
\end{tabular}

Table 3.13: Architectural requirements for efficient MD5 and SHA implementation 
MD5 processor. Applied to the $400 \mathrm{MHz}$ example this means a throughput of $924 \mathrm{MBit} / \mathrm{s}$ for SHA and $576 \mathrm{MBit} / \mathrm{s}$ for MD5.

Neither MD5 nor SHA need specific instructions although they would greatly benefit from monolithic non-linear functions or even monolithic round functions, but considering the already high throughput such specialized functions would clearly be overkill. Both algorithms show up a high level of parallelism and could use up to 5 parallel ALUs plus appropriate memory units providing the input values. The algorithms' requirements are summed up in Table 3.13.

\subsection{The International Data Encryption Algo- rithm (IDEA)}

The IDEA algorithm appeared in 1992 [73] and was the result of an ongoing improvement of the original Proposed Encryption Standard (PES) which was presented in 1990 and its successor the Improved Proposed Encryption Standard (IPES) [74, 72]. Due to patent reasons it never was as widely used as DES but gained popularity through the well-known and widely used PGP software [139, 138, 102] which is mainly used for email encryption and authentication.

Like DES, the IDEA algorithm is a symmetrical algorithm and operates on 64-bit data chunks. Contrary to DES, key size was doubled to 128 bit. Also round key generation for decryption is more complex. Figure 3.14 gives an overview of the IDEA algorithm.

The 64-bit input data is split up into four chunks of 16 bits which are fed into the first round of calculation. After 8 rounds the data undergoes an output transformation and the resulting four 16-bit chunks are concatenated to form the 64-bit output data.

A single computation round is depicted in Figure 3.15. Unlike DES which is mainly based on static permutation and XOR, IDEA was designed using "a mixture of operations from different algebraic groups" [101]. These operations are chosen to be efficiently implementable, both in hardware and software. Since the data chunks used for computation are 16 bits in size, IDEA is efficiently implementable on 16-bit architectures and does not prefer 32-bit architectures like AES/Rijndael or MD5. 


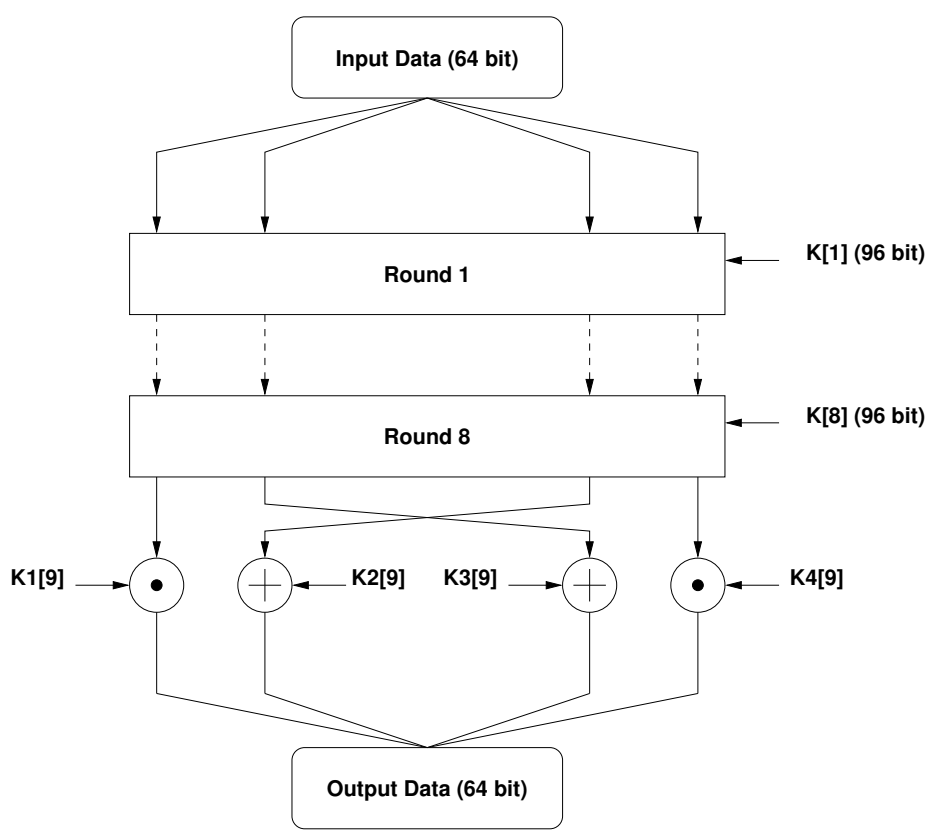

Figure 3.14: Workflow of IDEA

\subsubsection{Round Key Generation}

Round key generation for IDEA is fairly easy. The 128-bit key is divided into 8 sub-keys of 16 bits each. From these 8 sub-keys, the first six are applied as round keys named $Z_{1}^{(1)}$ to $Z_{1}^{(6)}$ to the first round, the last two are used as $Z_{2}^{(1)}$ and $Z_{2}^{(2)}$ for the second round. Now all 8 sub-keys of the first iteration are applied and the 128-bit key is rotated left by 25 bits and again divided into 8 sub-keys. The first four of this second iteration will be applied to round 2 as round keys $Z_{2}^{(3)}$ to $Z_{2}^{(6)}$, the remaining four go to round 3 as keys $Z_{3}^{(1)}$ to $Z_{3}^{(4)}$. Now the 128 -bit key is again rotated left by 25 bits and the distribution of sub-keys starts over again. This scheme is applied to all 8 rounds, the very last four sub-keys are used with the output transformation which makes a total of 52 sub-keys (cipher keys) to be used within IDEA.

Decryption is similar, however the keys are applied in reverse order; furthermore the round keys are partly different: For the even numbered round keys the additive inverse $-Z_{j}^{(i)}$ is used, for odd numbered round keys the 


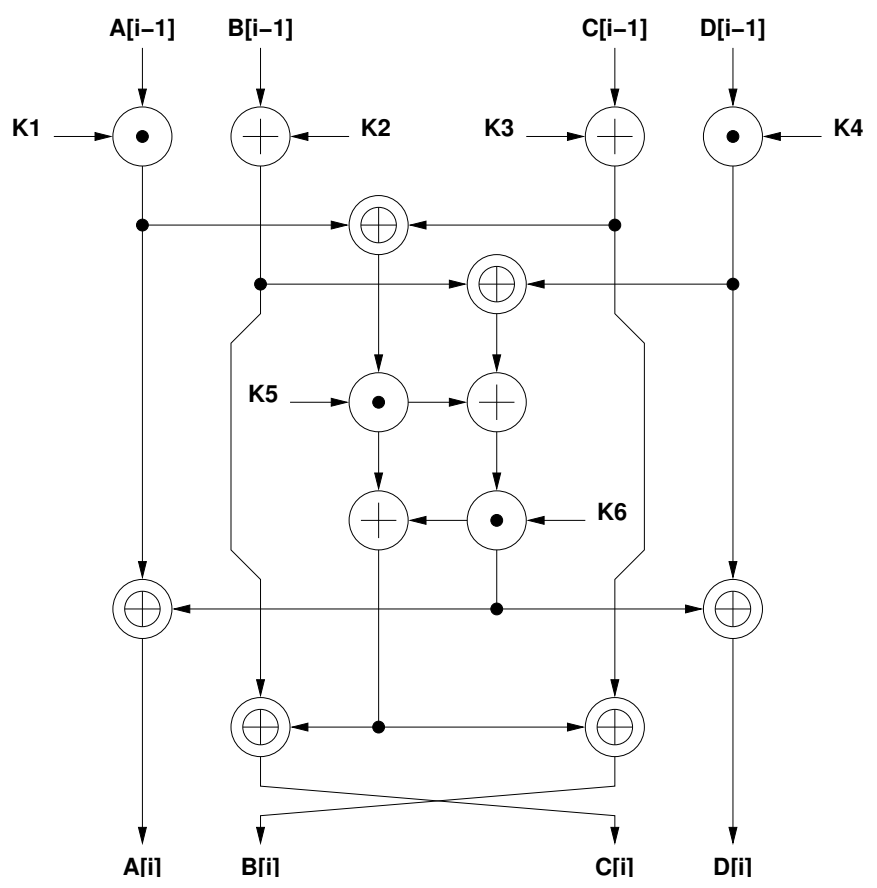

Figure 3.15: A single round of IDEA

multiplicative inverse $Z_{j}^{(i)-1}$ is applied. Like all computations within IDEA also the inversion is based on modulo $2^{16}+1$ arithmetics.

Table 3.14 shows the context between round number and round key generation for both, encryption and decryption.

\subsubsection{Algorithm Analysis}

IDEA was designed to run especially well on 16-bit hardware. Hence it is not surprising that all data chunk sizes used during computation are plain 16-bit - much unlike DES with its many different chunk sizes (28-, 32-, 48, and 56-bit), AES/Rijndael, or MD5 which were designed having today's 32-bit architectures in mind and operate only on 32-bit (long/int) and 8-bit (byte) quantities.

Much unlike these algorithms, IDEA makes heavy use of multiplications. To ideally support IDEA an architecture would need a 16x16-bit modulo $_{16}$ multiplier. Furthermore, for decipher key (decryption round key) 


\begin{tabular}{|c|ccccc|ccccccc|}
\hline Round & \multicolumn{9}{|c|}{ Encryption Round Key } & \multicolumn{5}{c|}{ Decryption Round Key } \\
\hline \hline 1 & $Z_{1}^{(1)}$ & $Z_{2}^{(1)}$ & $Z_{3}^{(1)}$ & $Z_{4}^{(1)}$ & $Z_{5}^{(1)}$ & $Z_{6}^{(1)}$ & $Z_{1}^{(9)-1}$ & $-Z_{2}^{(9)}$ & $Z_{3}^{(9)-1}$ & $-Z_{4}^{(9)}$ & $Z_{5}^{(9)-1}$ & $-Z_{6}^{(9)}$ \\
2 & $Z_{1}^{(2)}$ & $Z_{2}^{(2)}$ & $Z_{3}^{(2)}$ & $Z_{4}^{(2)}$ & $Z_{5}^{(2)}$ & $Z_{6}^{(2)}$ & $Z_{1}^{(8)-1}$ & $-Z_{2}^{(8)}$ & $Z_{3}^{(8)-1}$ & $-Z_{4}^{(8)}$ & $Z_{5}^{(8)-1}$ & $-Z_{6}^{(8)}$ \\
3 & $Z_{1}^{(3)}$ & $Z_{2}^{(3)}$ & $Z_{3}^{(3)}$ & $Z_{4}^{(3)}$ & $Z_{5}^{(3)}$ & $Z_{6}^{(3)}$ & $Z_{1}^{(7)-1}$ & $-Z_{2}^{(7)}$ & $Z_{3}^{(7)-1}$ & $-Z_{4}^{(7)}$ & $Z_{5}^{(7)-1}$ & $-Z_{6}^{(7)}$ \\
4 & $Z_{1}^{(4)}$ & $Z_{2}^{(4)}$ & $Z_{3}^{(4)}$ & $Z_{4}^{(4)}$ & $Z_{5}^{(4)}$ & $Z_{6}^{(4)}$ & $Z_{1}^{(6)-1}$ & $-Z_{2}^{(6)}$ & $Z_{3}^{(6)-1}$ & $-Z_{4}^{(6)}$ & $Z_{5}^{(6)-1}$ & $-Z_{6}^{(6)}$ \\
5 & $Z_{1}^{(5)}$ & $Z_{2}^{(5)}$ & $Z_{3}^{(5)}$ & $Z_{4}^{(5)}$ & $Z_{5}^{(5)}$ & $Z_{6}^{(5)}$ & $Z_{1}^{(5)-1}$ & $-Z_{2}^{(5)}$ & $Z_{3}^{(5)-1}$ & $-Z_{4}^{(5)}$ & $Z_{5}^{(5)-1}$ & $-Z_{6}^{(5)}$ \\
6 & $Z_{1}^{(6)}$ & $Z_{2}^{(6)}$ & $Z_{3}^{(6)}$ & $Z_{4}^{(6)}$ & $Z_{5}^{(6)}$ & $Z_{6}^{(6)}$ & $Z_{1}^{(4)-1}$ & $-Z_{2}^{(4)}$ & $Z_{3}^{(4)-1}$ & $-Z_{4}^{(4)}$ & $Z_{5}^{(4)-1}$ & $-Z_{6}^{(4)}$ \\
7 & $Z_{1}^{(7)}$ & $Z_{2}^{(7)}$ & $Z_{3}^{(7)}$ & $Z_{4}^{(7)}$ & $Z_{5}^{(7)}$ & $Z_{6}^{(7)}$ & $Z_{1}^{(3)-1}$ & $-Z_{2}^{(3)}$ & $Z_{3}^{(3)-1}$ & $-Z_{4}^{(3)}$ & $Z_{5}^{(3)-1}$ & $-Z_{6}^{(3)}$ \\
8 & $Z_{1}^{(8)}$ & $Z_{2}^{(8)}$ & $Z_{3}^{(8)}$ & $Z_{4}^{(8)}$ & $Z_{5}^{(8)}$ & $Z_{6}^{(8)}$ & $Z_{1}^{(2)-1}$ & $-Z_{2}^{(2)}$ & $Z_{3}^{(2)-1}$ & $-Z_{4}^{(2)}$ & $Z_{5}^{(2)-1}$ & $-Z_{6}^{(2)}$ \\
Output Xform & $Z_{1}^{(9)}$ & $Z_{2}^{(9)}$ & $Z_{3}^{(9)}$ & $Z_{4}^{(9)}$ & \multicolumn{10}{c}{$Z_{j}^{(i)}$ means the j-th sub-key of Round i } & & \\
\hline
\end{tabular}

Table 3.14: IDEA sub-keys used for encryption and decryption 


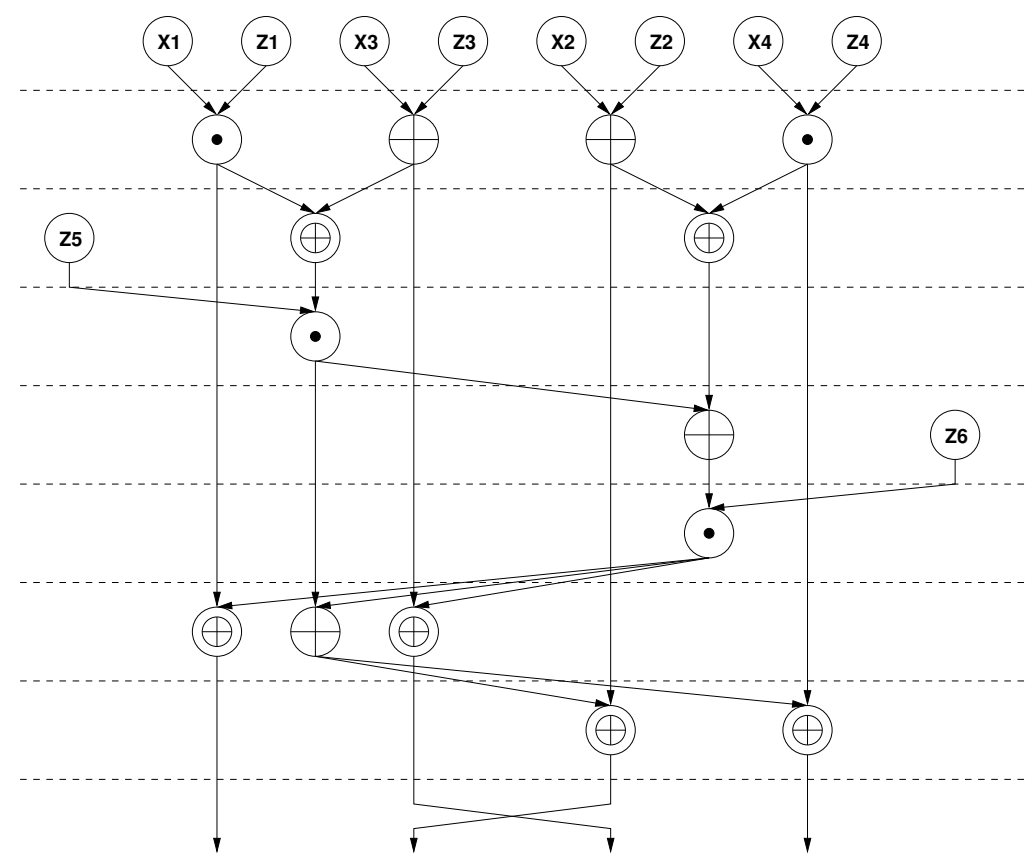

Figure 3.16: Data flow within an IDEA round

calculation division and modulo arithmetics based on modulo $2^{16}-1$ are needed. Although these operations can be emulated using just addition, subtraction, and Boolean operations, doing so would highly impact computation speed.

Assuming that the needed functions are present, data flow analysis of IDEA shows a quite high register pressure. Not less than 8 input variables are fed into the first calculation step where four of the results have to be kept for further processing in steps 6 and 7. Similarly, the results of step 2 and 3 have to be queued for later use.

Regarding parallelism, IDEA gives a quite unbalanced view as shown in Figure 3.16. For the very first step four algorithmical operations can happen in parallel; this drops down to two in step 2 and reaches strict sequentiality for steps 3 to 5 . The following two steps again incorporate 3 and 2 parallel operations, finally a swap operation between two results takes place and the next round begins. For the eighth round the final swapping is omitted, 


\begin{tabular}{|c|c|}
\hline Parallelism & $\begin{array}{l}\text { - Maximum parallelism of } 4 \text { instructions per cy- } \\
\text { cle; ideally, either } 4 \text { memory operations, } 4 \text { al- } \\
\text { gorithmic operations or mixtures of both } \\
\text { - Round key generation can be embedded into se- } \\
\text { quential part of round calculation }\end{array}$ \\
\hline Instruction Set & $\begin{array}{l}\text { - } 128 \text {-bit rotation for round key generation } \\
\text { - single-cycle 16x16-bit multiplication } \\
\text { - single-cycle 16:16-bit division and modulo op- } \\
\text { eration for decipher key generation }\end{array}$ \\
\hline Data Size & $\begin{array}{l}\text { - Input/Output: } 64 \text { bit (Data), } 128 \text { bit (Key) } \\
\text { - Intermediate Results: } 16 \text { bit }\end{array}$ \\
\hline
\end{tabular}

Table 3.15: Architectural requirements for efficient IDEA implementation

instead the output transformation, four parallel arithmetic operations, take place.

Thus, an ideal IDEA processor could process a complete encryption or decryption within $1+8 * 8=65$ cycles which equals a processing rate of $\frac{64 \text { bits }}{65 \text { cycles }}=0.99$ bits per cycles or 1.01 cycles per bit which is about twice the numbers as calculated for DES, which also operates on 64-bit data chunks. Assuming that this IDEA processor runs at $400 \mathrm{MHz}$ it will reach a throughput of about $394 \mathrm{MBit} / \mathrm{s}$ as opposed to $775 \mathrm{MBit} / \mathrm{s}$ for DES.

\subsection{The RC6 ${ }^{T M}$ Block Cipher}

$\mathrm{RC}^{3}$ was one of the competitors for becoming the new AES standard and is a successor to the RC5 Block Cipher[94, 95, 109]. It was not only designed to meet the AES committee's requirements but also to be ideally suited for today's 32-bit microprocessors.

\footnotetext{
${ }^{3} \mathrm{RC} 6$ is a registered trademark of RSA Laboraties
} 
Like RC5, RC6 is parametrizable and should correctly be written RC6$w / r / b$ where $w$ denotes the size of data chunks in bits, $r$ number of rounds and $b$ the length of the encryption key in bytes which can be any number between 0 and 255. For AES submission, RC6 was configured with $w=32$, $r=20$ and $b=16$. With these parameters, key and block size of RC6 correspond to Rijndael-128/128. Regardless of configuration data, RC6 always employs four working registers of $w$ bits size and six basic operations which are listed in Table 3.16.

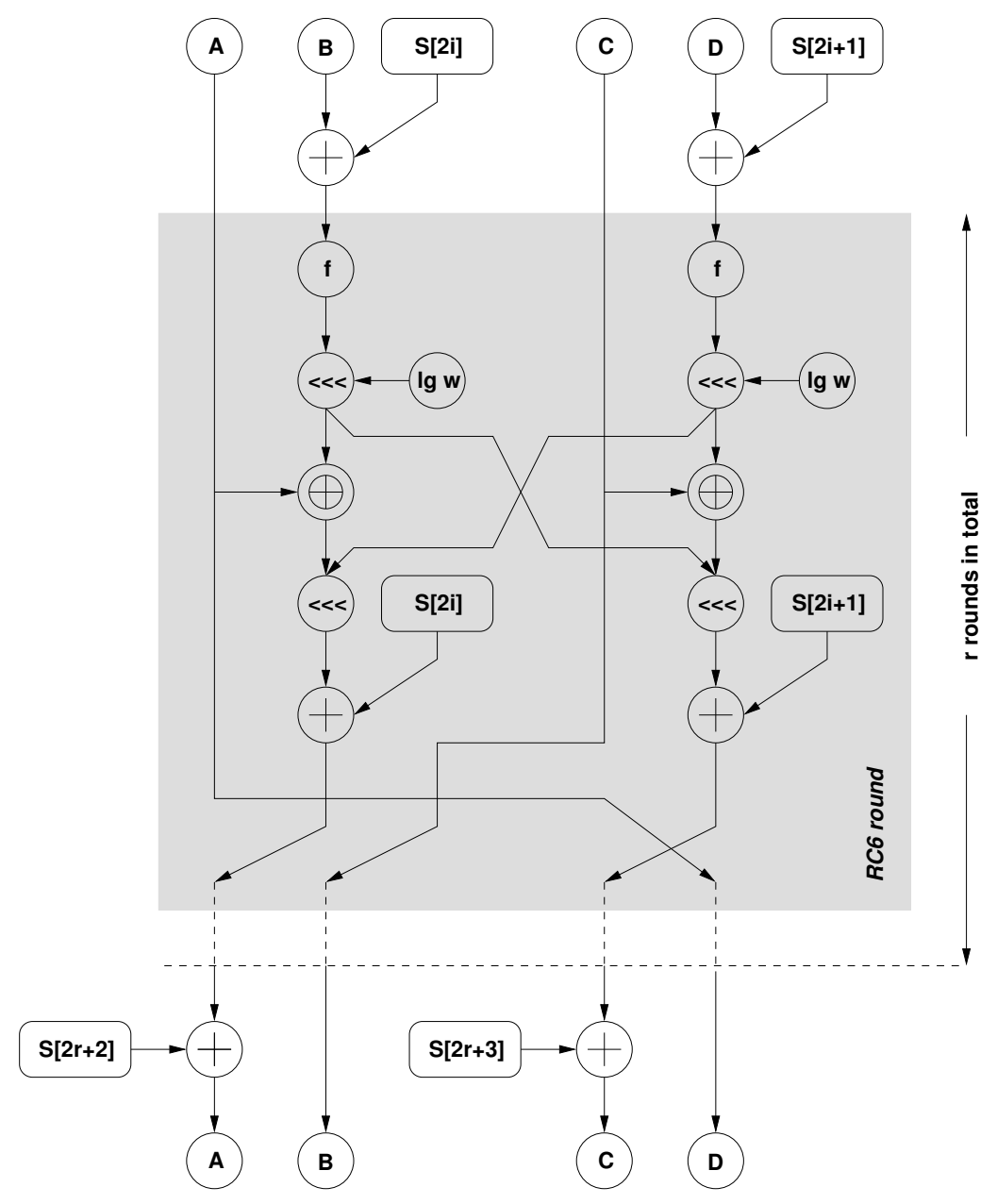

Figure 3.17: Workflow of the RC6 ${ }^{T M}$ Block Cipher 


\begin{tabular}{|ll|}
\hline$a+b$ & integer addition modulo $2^{w}$ \\
$a-b$ & integer subtraction modulo $2^{w}$ \\
$a \times b$ & integer multiplication modulo $2^{w}$ \\
$a \oplus b$ & bitwise exclusive-or of $w$-bit words \\
$a \lll b$ & $\begin{array}{l}\text { rotate the } w \text {-bit word } a \text { to the left by the amount given } \\
\text { by the least significant } \log _{2} w \text { bits of } b\end{array}$ \\
$a \ggg b$ & $\begin{array}{l}\text { rotate the } w \text {-bit word } a \text { to the right by the amount } \\
\text { given by the least significant } \log _{2} w \text { bits of } b\end{array}$ \\
\hline
\end{tabular}

Table 3.16: RC6 Basic Operations

\subsubsection{Key Scheduling}

Key scheduling of RC6 is practically identical to key scheduling of RC5: As said before, any key $b$ consists of $0 \leq b \leq 255$ bytes. From this key, $2 r+4$ words of $w$ bits each are generated and stored in the round key array $S[0, \ldots, 2 r+3]$.

The key scheduling consists of two loops, an initialization loop which presets the round key array based on distinct initialization values, and the main computation loop which generates the round key array by severe mixing of the supplied user key [96]. Compared to other algorithms, RC6's key generation is rather heavyweight and makes it sensible to precompute the round key array. This eventually causes performance issues as illustrated in [97]. Implementations on smaller processors or in hardware might suffer from the use of multiplication and modulo operations for round key generation.

\subsubsection{Algorithm Analysis}

Like all other algorithms discussed so far also RC6 is based on rounds. As depicted in Figure 3.17, RC6 consists of an initial addition of the first two round keys to the input variables $B$ and $D$ followed by $r$ rounds. Finally, the $A$ and $C$ results are added to the $2 r+2^{n d}$ and $2 r+3^{r d}$ round key.

For decryption the algorithm is just processed backwards starting with keys $2 r+2$ and $2 r+3$ being added to $A$ and $C$ followed by $r$ rounds and ending with the addition of key 0 and 1 to $B$ and $C$. In this manner it 


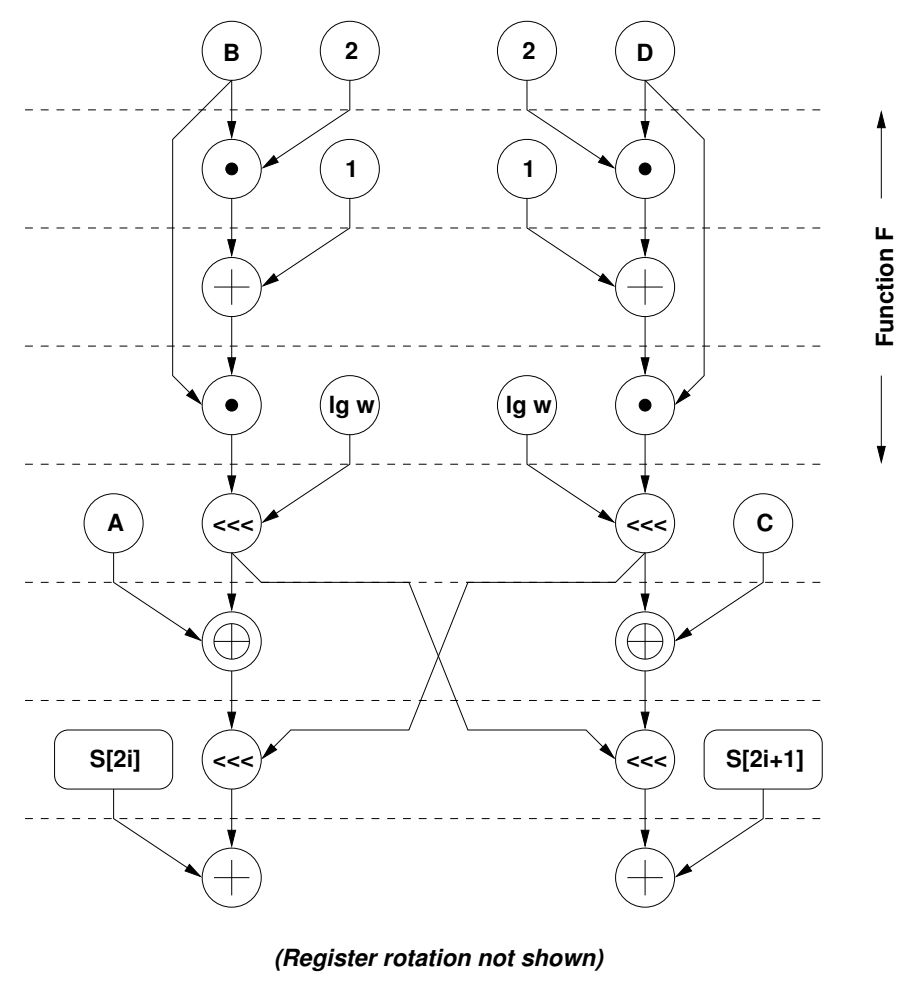

Figure 3.18: Data flow within one $\mathrm{RC}^{T M}$ round

resembles much of DES if you consider these key additions to be input and output transformation.

Basically, each round consists of two identical $>$ strands which results are intermixed after the shift operation. There is a strong data dependency within each strand which makes it impossible to combine or precompute intermediate results as shown in Figure 3.18 which means, that there is again much like DES - not much parallelism to exploit. The round key generation is pretty trivial as shown in 3.8.1 and can be reduced to memory lookups.

This leads to technology requirements as shown in Table 3.18 with the speed estimations listed in Table 3.17: For AES configuration ( $w=32$ bits, $r=20$ rounds, $b=16$ byte $=128$ bits) this should give a performance of $2+$ $(20 * 7)+1=143$ cycles equalling 0.90 bits per cycle or 1.12 cycles per bit without having the monolithic RC6-specific function. On an estimated 
$400 \mathrm{MHz}$ clock rate this will lead to a throughput of $358 \mathrm{MBit} / \mathrm{s}$. If the RC6-specific function is present, the numbers are $2+20 * 4+1=83$ cycles equalling 1.54 bits per cycle or 0.65 cycles per bit leading to a throughput of almost $617 \mathrm{MBit} / \mathrm{s}$ at $400 \mathrm{MHz}$.

$\begin{array}{ll}\text { Initialization } & 2 \text { cycles } \\ \text { Round Processing } & 7 / 4 \text { cycles } \\ \text { Postprocessing } & 1 \text { cycle }\end{array}$

Table 3.17: Speed estimations for RC6 on an ideal architecture

\begin{tabular}{|c|c|}
\hline Parallelism & $\begin{array}{l}\text { - Arithmetic operation can happen in parallel to } \\
\text { memory lookup (round key) } \\
\text { - RC6 consists of two strands with sequential na- } \\
\text { ture; thus, two arithmetic operations can be pro- } \\
\text { cessed in parallel }\end{array}$ \\
\hline Instruction Set & $\begin{array}{l}\text { - Addition and Multiplication modulo } 2^{w} \\
\text { - Exclusive-OR on operands of } w \text { bits } \\
\text { - Rotate-Left by } \log _{2} w \text { positions on } w \text {-bit } \\
\text { operand } \\
\text { - Register Swapping } \\
\text { - Special instruction } f(x)=x *(2 x+1) \lll \log _{w} \\
\text { would save } 3 \text { cycles per round }\end{array}$ \\
\hline Data Size & $\begin{array}{l}\text { - Input/Output: } w * 4 \text { bit, typically } 128 \text { bit }(w=32) \\
\text { - Intermediate Results: } w \text { bit }(32,48 \text {, or } 64 \text {-bit } \\
\text { operand size) }\end{array}$ \\
\hline
\end{tabular}

Table 3.18: Architectural requirements for efficient $\mathrm{RC}^{T M}$ implementation 


\subsection{Summary}

In this chapter, a selected set of crypto algorithms was presented. These are namely DES and 3DES, AES/Rijndael, IDEA and RC6. Also, a number of hash algorithms used for message authentication were investigated: The widely used MD5 and its somewhat improved version SHA-1 were selected; furthermore, the relationship to their common predecessor, MD4, was illustrated. Also, the influence of selected algorithm and operation mode on possibly exploitable parallelism was explained.

The algorithms were selected for many reasons. DES is not only the first standardized crypto algorithm but is also very hardware-oriented, In contrast, AES/Rijndael, the new standard, was designed with respect to actual 32-bit architectures but can be also realized on less powerful architectures. IDEA stands somewhat in between these two algorithms since it was designed for 16-bit architectures but requires fast modulo-multiplication and -division units. Finally, RC6 was selected, since it was one of the most promising candidates in the AES competition. The hash algorithms were selected to include a different algorithm class into this investigation to work out a most general architecture.

Where this chapter concentrated more on the needs of the single algorithms and how they can be implemented in an ideal manner, their impact on the CRYPTONITE architecture will be elaborated in Chapter 4. 
3. Algorithm Discussion AND ANALYSIS 


4

\section{Architecture Impact resulting from Algorithm Analysis}

In the previous chapter a set of well known and widely used algorithms was discussed. These algorithms were selected to cover a broad range of possible applications and included crypto algorithms designed for hardware implementation (represented by DES and 3DES), and these developed to run efficiently on 16-bit (IDEA) and 32-bit (AES/Rijndael, RC6) general purpose processors. Also hashing algorithms, MD5 and SHA, were included.

Each algorithm has certain architectural needs. Some algorithms would greatly benefit from three-input functions, others require special monolithic functions for high performance. However, in a programmable environment algorithm-specific functions have to be reduced to an absolute minimum since the idea of such an architecture is certainly not building a container around dedicated hardware solutions.

Based on the results of the previous chapter the influences on the architecture will be investigated by determining the average requirements of all explored algorithms. This examination will then provide information about the basic architectural concepts like number and type of functional units, register size and count, and the necessary instruction set.

\subsection{Functional Units}

Analyzing the algorithms with respect to used operations results in the list printed in Table 4.1. Concerning the XOR functions two observations can 
- Boolean operations like logical AND, OR and XOR; also a NOT operation for inverting an operands bit pattern is needed as a result of the hash algorithm analysis

- simple $\operatorname{modulo}_{w}$ algorithmic operations like addition, subtraction

- complex $\operatorname{modulo}_{w}$ algorithmic operations like multiplication, division, and modulo as needed by the IDEA algorithm

- single indexed memory access for loading round constants and storing results

- simple load/store memory access for keeping and reloading intermediate results

- multiple parallel indexed memory accesses as needed for S-Box lookups

- conditional looping of code snippets for repeating computation rounds without the need of loop unrolling which would lead to bloated code size

Table 4.1: Operations employed in analyzed algorithms

be made: First, there are certain circumstances where especially the XOR function could use more than one input, for instance within one of the nonlinear functions of MD5 and SHA which ideally make use of a 3-input XOR function. Second, the XOR function often is the final step after a series of computations. Ideally, this final step could happen in parallel to other algorithmic operations to speed up computation. This consideration leads to a somewhat partite ALU consisting of a register file for parameter storage, an XOR unit and an arithmetic unit as described in Section 5.7.

For memory access a very basic architecture was considered: No immediate memory access is possible, but memory access is always registerbased which means that a memory address has first to be loaded into an address register; memory access can then happen based on the content of a selected address register. Extending this model by a simple adder logic 
enables combining two address registers; this leads to indexed memory addressing. This scheme can be modified to allow parallel S-Box access as described in Section 5.8.1 when discussing the address generation unit. The complete memory unit is presented in Section 5.8.

Since some of the crypto algorithms clearly separate into two strands as shown with DES (encryption and key generation), IDEA or RC6 (two parallel strands interchanging values within encryption) it seems to be somewhat natural to realize two individual sets containing one ALU and memory unit. This reproduces the observed two-strand behaviour in hardware. This basically means just doubling ALUs, associated memory units and data memories which leads to a structure as described in Section 5.3. This structure also allows the architecture to exploit parallelism found within the other algorithms and leads to fast execution times as shown in Chapter 6.

This split architecture, however, creates a problem of exchanging values between the two strands as needed for all discussed algorithms. Here, a bipartite approach has been taken making it possible to forward computated results or register values from one ALU to the other as shown in Section 5.7

\subsection{Register File}

Registers are needed to hold input and output data as well as intermediate results. For that reason they need to satisfy a wide range of constraints such as data size and number of values to hold.

Not supporting certain data sizes would eventually result in additional cycles needed for modulo arithmetics or masking out unused bits. Supporting too many data sizes would unnecessarily add complexity to the design resulting in slower processing speed and higher power consumption.

Similarly, implementing too few registers will create a cycle penalty for intermediate results to be stored to or fetched from memory; too many registers, however, will increase the processor's die size, cause higher power consumption and potentially slower processing speed.

\subsubsection{Register Size}

Looking at the algorithms, the common data chunk size is 32-bit which is found within many of the discussed algorithms. Both hash algorithms use it, 
also AES/Rijndael and RC6 in AES configuration work on 32-bit entities. IDEA, however, is strictly 16-bit based.

A second common data chunk size is 8-bit which is commonly used for table (S-Box) lookup e.g. within AES/Rijndael and DES, the latter being quite special here since it takes 64-bit quantities but internally operates on a broad variety of bit sizes like 56-bit after input and before output transformation, 28-bit for key generation, 48-bit for S-Box lookup and 32-bit for intermediate round results. However, a closer look reveals, that these sizes can be mapped either to 32 or 64 bits by rearranging the "odd" sizes to 8or 32-bit boundaries. That way the 28-bit value would refer to an aligned 32-bit value, similarly the 48-bit data size as appearing together with the S-Box lookup can be rearranged to a 64-bit value where the 6-bit chunks are aligned to 8-bit boundaries.

A software implementation of DES following these alignment schemes and hence requiring only data sizes of 32- and 64-bit displayed no negative side-effects concerning registers. For this reason and since 8-bit data size is only employed within an S-Box lookup as mentioned above the decision was made to support only two data sizes: 64-bit (word), and 32-bit (half-word where upper and lower half-word of a 64-bit word are directly accessable). To select these data sizes a 2-bit control value is needed resulting in 4 possible states. The fourth, unoccupied, state is used to clear a register or zero its output ("muting" the register), allowing an easy reset of the register contents to zero as described in Section 5.7.2 and illustrated through Table 4.2.

\subsubsection{Number of Registers}

Looking at the algorithms shows that most of these incorporate just twoinput functions; this is certainly true for DES, RC6 and IDEA. Also most operations used for hashing only have two input parameters. Additionally, a straightforward implementation of AES/Rijndael shows that two-input functions are sufficient.

This means, that for a simple calculation step only two source registers are needed; some algorithms like IDEA or RC6 need to keep intermediate results for later computation steps so having an intermediate result register like an accumulator placed inside the ALU would come in quite handy. 


\begin{tabular}{|c|l|}
\hline Control Value & \multicolumn{1}{|c|}{ Operation } \\
\hline \hline $00_{2}$ & clear or "mute" register \\
$01_{2}$ & load 64-bit value into register \\
$10_{2}$ & load lower 32-bit half of register \\
$11_{2}$ & load upper 32-bit half of register \\
\hline
\end{tabular}

Table 4.2: Register Size Control

Three registers already need two address lines for access control which means that a fourth register comes in virtually for free in terms of system control. Such a fourth register will be quite useful on algorithms with great register pressure like AES/Rijndael or IDEA and can be used for storing multiple intermediate results or accelerate operations by pre-loading needed values.

Following this argument the number of registers per register file has been set to four as a compromise between register pressure caused by registerhungry algorithms like IDEA or AES/Rijndael and on-chip space requirements caused by the registers itself and their address and data paths.

Releasing this pressure by the possibility of exchanging values between registers of the two strands is a beneficial side effect of the interlink structure already described in Section 4.1. In addition, pressure is further eased by the possibility to use the Memory unit's data output register, which is needed for synchronizing data, as a supplementary source and destination registers for arithmetic operations as described in Section 5.8.

\subsection{Special Instructions}

A programmable device should not be a collection of specialized hardware. Instead, it should be based on ideally primitive and reusable hardware functions.

For some algorithms, however, the fully programmable approach does not make sense when it comes to computation speed: DES is a good example of an algorithm which has to be clearly based on specialized hardware. Of course, all DES operations can be reduced to a series of primitive operations such as Boolean algebra functions or shift operations, but this leads to 
a highly ineffective code. As a result of the DES analysis there clearly has to be a specialized DES unit to support this algorithm at reasonable speed as discussed in Section 5.8.4. AES/Rijndael is another example since both, its basic [46] and fast [45] implementations clearly need supportive operations to enable the desired speed requirements. However, unlike DES these operations as described in Section 5.7.3.1 do not need to be Rijndael-specific but could possibly be used for other applications.

It can be observed, that for efficient implementation especially the two standardized crypto algorithms show great need for specialized functions. In contrast, the other algorithms can easily be realized using Boolean and basic mathematical operations. Due to the continued use of (3)DES in many real-time applications such as (de)scrambling digital pay TV and the expected success of AES, operations supporting these algorithms must be implemented regardless of whether they can be re-used for other algorithms or not.

IDEA and RC6 use modulo-based multiplication which makes them quite different from all other discussed algorithms which only rely on table lookups, simple arithmetics, and boolean operations. Implementing a hardware multiplier would support these algorithms and enhance computation speed greatly. However, the problem arises that IDEA uses $\bmod _{16}$ multiplication where RC6 favours $\bmod _{32}$ to $\bmod _{64}$ multiplication depending on the configuration. The solution to this dilemma with respect to the already taken decision towards a 64-bit architecture would be a configurable multiplier which either works as 64-bit*64-bit $\bmod _{64}, \bmod _{32}$, or $\bmod _{16}$ multiplier. Integer division and modulo operator, however, should not be included: With the investigated algorithms these operations are only needed together with IDEA round key calculation for decryption. The construction rules of these keys, however, disable embedding the round key generation into the encryption routine. Instead, they should be precomputed by external means and applied together with the input data.

\subsection{Summary}

Where Chapter 3 focused on the algorithms and an ideal architecture for each algorithm, this chapter concentrated on shaping out an architecture which serves well for a broad variety of algorithms. 
For this reason, the needs of all algorithms investigated in Chapter 3 was summed up and discussed with respect to their needs towards number and type of functional units, number and size of internal registers. Also, algorithm-specific instructions have been discussed.

Based on this discussion, an initial architecture partitioning into an arithmetic unit consisting of arithmetic-logical unit (ALU), XOR unit (XU) and associated register file, and a memory unit (MU) being responsible for data transfers from an associated data memory to the registers and vice versa was developed. A decision was made to incorporate two independent strands consisting of these units since many algorithms showed a certain amount of inherent parallelism which can be exploited by this two-strand model.

Furthermore, the register file has been defined as a set of four 64-bit registers with directly addressable 32-bit halves; to release register pressure and to allow exchange of values between the two strands, an interlink mechanism was created which enables forwarding of register values and computation results between the two independent strands.

Finally, a set of more or less algorithm-specific instructions was discussed. As for DES this resulted in a completely independent DES unit since DES operations are too special to be used for any other algorithm; using less specific operations, however, would lead to a inacceptable slowdown of DES performance. For AES/Rijndael a more unspecific set of supporting instructions has been defined. The presence of a $\bmod _{w}$ multiplication unit is recommended for IDEA and RC6, but can be omitted fue to the low distribution and use count of these two algorithms.

Chapter 5 will now describe the resulting architecture in detail. Please notice that due to signed contracts the work presented there is intellectual property of Agere Systems, USA. 
4. ARCHITECTURE IMPACT RESUlting From AlgORIthM ANALYSiS 


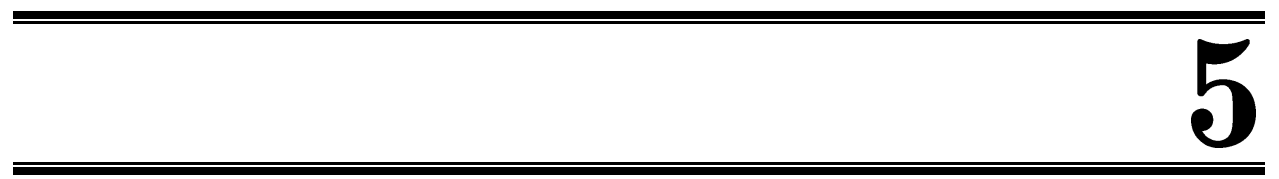

\title{
The CRYPTONITE Architecture
}

According to contracts signed by the author the work presented in this chapter is intellectual property of Agere Systems, USA, with patents pending. With permission of Agere Systems the author is allowed to publish this chapter publicly.

If you plan to use the architecture described in this chapter or parts of it commercially, please contact Mr. Nevin Heinze of Agere Systems. He can be reached as follows

\author{
Agere Systems \\ Processor Architectures \& Compiler Research \\ Mr. Nevin Heintze \\ 4 Connell Drive, Room 4P-739 \\ Berkeley Heights, NJ 07922 \\ U.S.A.
}

Alternatively, you can reach Mr. Heintze via e-mail (ncheagere.com). 


\subsection{Design Goals}

To understand how this architecture was designed the following design criteria have to be taken into account which are

- Technology limitations The most critical design parameters for the CRYPTONITE architecture are single-cycle access to registers and onchip memory and single-cycle execution of the implemented arithmetic operations to enable maximum processing speed. For technology reasons, namely single cycle access to memory, the operating frequency was limited to $400 \mathrm{MHz}$. Also, pipelining techniques have to be used since it is infeasible that instruction fetch, decoding, and execution can happen within one $400 \mathrm{MHz}$ cycle.

- Due to bandwidth demands, however, an ideal architecture should be able to cope with a summarized data bandwidth of $4 \mathrm{GBit} / \mathrm{s}$. Since this is definitely not achievable with a single, fully programmable processor element a per-element throughput of $500 \mathrm{MBit} / \mathrm{s}$ was defined. A production system should then achieve the denoted $4 \mathrm{GBit} / \mathrm{s}$ throughput by employing multiple processor elements serving independent data streams.

- These hard parameters, $500 \mathrm{MBit} / \mathrm{s}$ throughput at $400 \mathrm{MHz}$ resulting in a relative speed of 1.2 bits per cycle or 0.8 cycles per bit, propagate a parallel architecture with very tight timing constraints. This means that functional units have to be designed in a way that the overall signal latency must still fit into a single cycle.

These targets are not only independent of the crypto algorithms, which in turn define which functional units are needed in what configuration, but also have higher priority which means that the proposed architecture is a trade-off between technological specifications and algorithm demands: For example, throughput issues dictate a rather high bits-per-cycle ratio demanding a single-cycle execution model to guarantee maximal throughput. Economical issues limit the frequency to $400 \mathrm{MHz}$ where technology issues make a pipelined architecture mandatory to ease hardware parameters but still be able to provide necessary computation speed. 


\subsection{General Purpose Architectures - An Alter- native?}

Today's high-performance general purpose architectures (GPAs) like the Intel Pentium or Itanium families represented through Pentium 4 and ItaniumII are able to deliver an enormous computation power. The decision to use a dedicated architecture at first glance does not seem to be justified for the following reasons:

- Modern GPAs are superscalar architectures and offer computation speeds up to $2.5 \mathrm{GHz}$

- They are mass products resulting in comparably low prices; also, numerous development systems exist for these processors which programmers are used to.

- Similarly, readily developed interface hardware like PCI bridges, even complete hardware infrastructures - main or mother boards - exist in many configurations which decrease development time significantly.

However, despite these quite convincing facts, there are also reasons against using GPAs:

- Despite their computation power, GPAs still are not able to compete with dedicated processors for certain applications. This is especially true for cryptography. Based on the numbers given in [47] and [110] cycle count of software implementations based on GPAs is still an average factor of 7.55 higher than cycle count for the same algorithms implemented on the CRYPTONITE architecture. In worst case (AES/Rijndael decryption including round key generation) the performance gap reaches even a factor of 21.76 .

- High-performance GPAs have an enormous power consumption. For example, members of the Pentium-4 family running at $2.53 \mathrm{GHz}$ use nearly 70 Watts [35]. For this reason, these processors produce a tremendous amount of heat which requires either huge coolers or in case space matters - complicated and expensive cooling systems. 
The chipsets necessary to interface these processors to RAM and peripheral buses, the Memory Controller Hub (MCH) [33] and I/O Controller Hub (ICH) [32], consume another 11 Watts and therefore produce also a noticeable amount of heat. These two factors, power dissipation and heat generation, make these processors almost unusable for embedded systems.

- These processors only provide highest performance if the problem to be computed can be entirely held in cache. If accesses to external RAM are needed, they have to be reordered to hide RAM access latencies. As the investigated algorithms show very tight inner loops there is not much - depending on the algorithm even almost no - time to hide RAM access latencies. Due to the real-time character of the processed data streams it is neither possible to preload a bigger set of data in advance.

- Although the GPAs are comparably low in price, they need special interface chips (within the PC market referred to as"chipset") to make them work together with SDRAM or RDRAM and standardized peripheral buses like PCI. Typically, such a system consisting of main board holding the chipset together with a decent GPA running at 2.5 GHz will cost around 1000 Euro as of August 2002.

For the above reasons the development of a specialized architecture is justified: It will provide the necessary computation speed but will only consume a fraction of the power a current GPA needs and therefore produce much less heat. In addition, due to the lesser chip complexity the fabrication costs are expected to be much lower.

\subsection{Architecture overview}

CRYPTONITE as shown in Figure 5.1 can be divided into two strands each consisting of an Arithmetic Unit (see Section 5.7) and its corresponding Memory Unit (see Section 5.8) controlling the associated data memory. The Memory Unit itself separates into an Address Generation Unit (AGU) being responsible for address generation based on the selected addressing mode (Sections 5.8.1 and 5.8.2) and a Data Input/Output Unit (DIO) (Section 


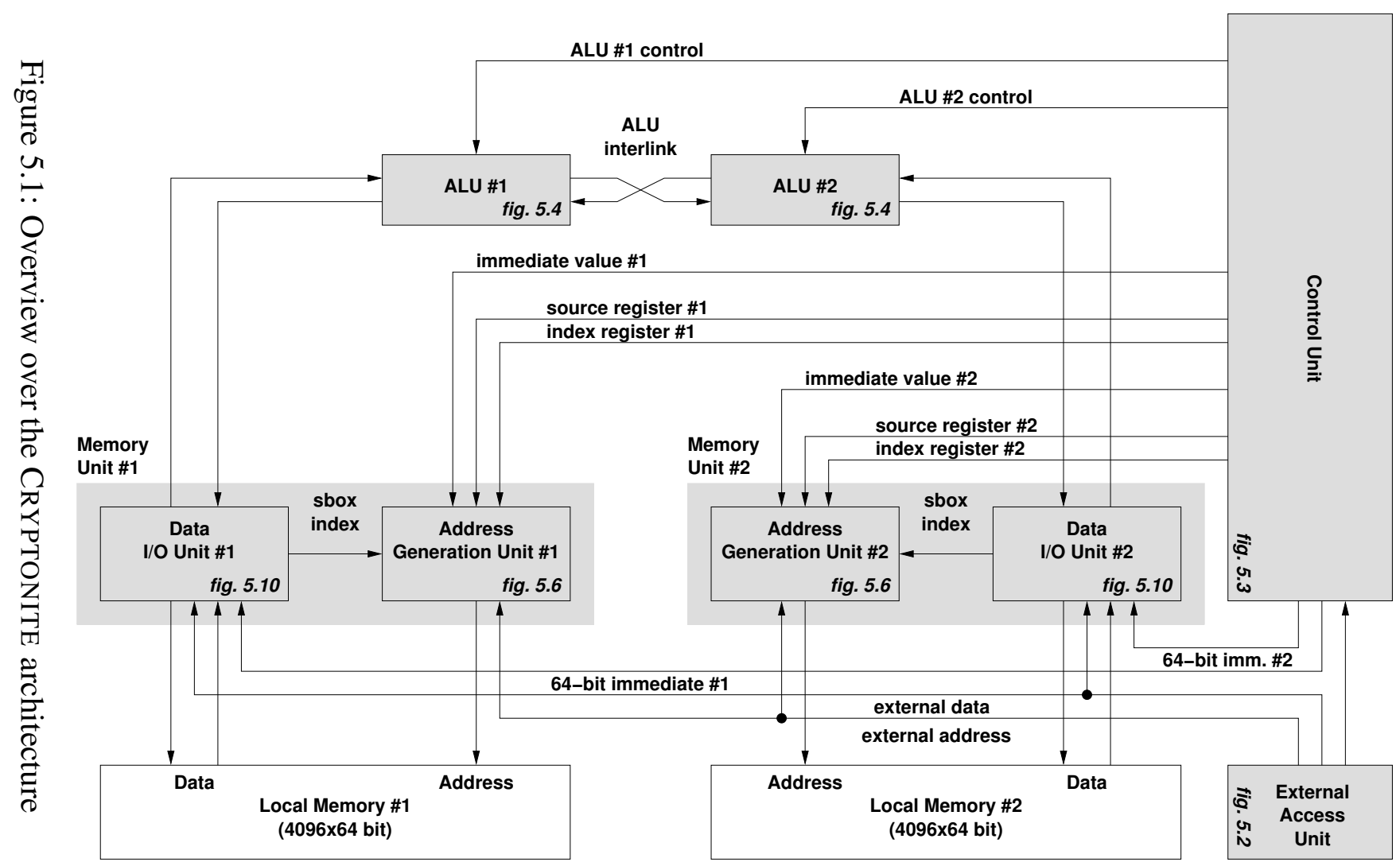


5.8.3) where the latter holds also a DES-specific unit (Section 5.8.4).

The following architecture presentation will concentrate on single units. The reader should keep in mind that whenever the text refers to ALU registers, local address registers or data input/output registers these register sets are individually present within each strand.

\subsection{The Pipeline}

CRYPTONITE is targeted towards $400 \mathrm{MHz}$ operating frequency providing single-cycle execution. For this reason, the architecture needs to be pipelined since it is not realistic to assume that instruction fetch, decoding and execution can happen within one $400 \mathrm{MHz}$ cycle. Theoretically, the pipeline could be hidden if the $400 \mathrm{MHz}$ clock is internally tripled resulting in an internal clock frequency of $1.2 \mathrm{GHz}$. Doing so would, however, cause too high requirements towards chip hardware and design.

\begin{tabular}{|c|c|c|}
\hline Stage & Function & Function \\
\hline$\overline{1}$ & $\begin{array}{r}\text { Instruction } \\
\text { Fetch }\end{array}$ & $\begin{array}{l}a d d r \leftarrow P C \\
P C \leftarrow P C+1\end{array}$ \\
\hline 2 & $\begin{array}{r}\text { Instruction } \\
\text { Decode }\end{array}$ & $\begin{array}{l}\text { create internal control signals } \\
P C \leftarrow \text { immediate address } \\
\text { (PC assignment for immediate } \\
\text { branches only) }\end{array}$ \\
\hline 3 & $\begin{array}{r}\text { Execution \& } \\
\text { Writeback }\end{array}$ & $\begin{array}{l}\text { perform operations } \\
P C \leftarrow \text { immediate address } \\
\text { (PC assignment for conditional } \\
\text { branches only) }\end{array}$ \\
\hline
\end{tabular}

Table 5.1: Pipeline Stages of the CRYPTONITE Architecture

For this reason, a three-stage pipeline is employed as explained in Table 5.1. Besides releasing timing pressure from hardware, the proposed instruction pipeline motivates the use of delayed branches as also shown in Table 5.1. Immediate branches have a one-cycle delay and become effective after pipeline stage 2 (instruction decode); for conditional branches the delay is 
2 cycles since these have to wait for the result of the execute stage which means that the new PC value - in case the conditional branch was taken becomes effective after pipeline stage 3. Already fetched instructions will be processed during the delay cycles.

Branch delay is a characteristic which applies to all pipelined architectures and is therefore known from several architectures like the industrially manufactured AMD AM29K [4] or the educational DLX processor designed by Hennessy \& Patterson [52]: Depending on the pipeline length and the branch delay caused through this, one or more instructions following a branch instruction are executed.

For typical crypto algorithms as discussed in Chapters 2 and 3 the control flow is usually not data driven which makes it easy to rearrange code avoiding increase in cycle count or code size. In most cases branch delay can be circumvented by code rearrangement, only in very few situations code increase applies: These are usually loops with bodies smaller than 3 instructions. To avoid padding NOP instructions - which negatively affect throughput - loops of this type have to be partially unrolled to allow code rearrangement.

\subsection{Access from External Devices}

External access to CRYPTONITE is necessary to enable updates of internal look-up tables as needed for external computation of round keys and also to feed in data chunks and key updates within a running computation as well as reading back result data. The physical interface between CRYPTONITE and an external device is realized through the External Access Unit (EAU) as depicted in Figure 5.2.

Whenever an external access to local data memory happens, the requested address plus the actual content of the external data bus is sampled; at the same time a ready flag is cleared to signal to the external unit that CRYPTONITE is now busy with the requested data transfer. This flag is also fed into the Control Unit (see Section 5.6). Internally, a maskable interrupt is triggered which stops current execution and performs the requested memory operation by inserting 3 cycles into the pipeline as shown in Table 5.2. This kind of external access interrupt is enabled by default but can 


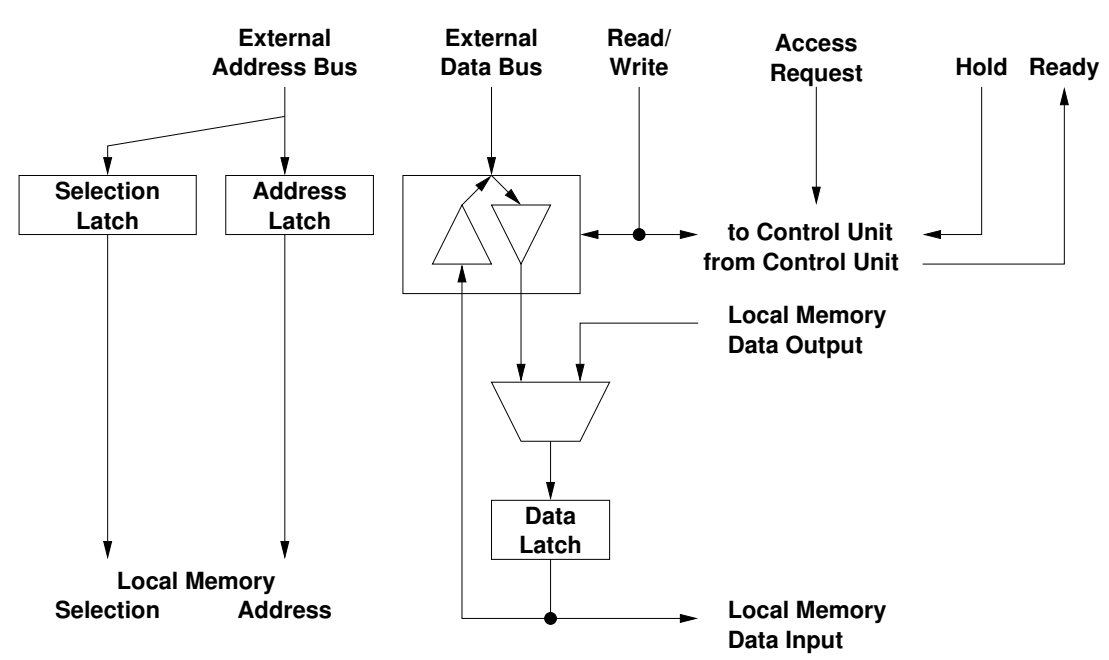

Figure 5.2: The External Access Unit

be disabled using the CU's DI command. To re-enable interrupts, the EI command is used.

Within the first cycle, the sampled address is put on the local memory address bus; in case of write access, the sampled data is transferred into the data input register, which in term is saved to a shadow register. During the second cycle, the requested data transfer happens and - in case of a read access - the return data is sampled in the data output register. In this case, the previous value of the data output register is saved to a shadow register. In the third cycle, the data input and output registers are restored while the content of the data input register is transferred to the external data register and the ready flag is risen.

To allow synchronous transfer, a hold input signal exists. Whenever the CU encounters a HOLD command, it puts further execution on hold by assigning NOP operations to all units and not incrementing the program counter until the hold input signal raises from low to high level. Due to the employed pipeline, HOLD will show the same delay as unconditional branches as explained in Section 5.6.2.

This approach was taken to provide a method of accessing local data memory and synchronizing CRYPTONITE to external devices with minimal impact to the core architecture. More heavyweight interface structures being 


\begin{tabular}{|c|l|l|}
\hline Cycle & Name & \multicolumn{1}{c|}{ Function } \\
\hline \hline 1 & Rescue & DIR $\rightarrow$ DIR $R_{\text {shadow }}$ \\
& \& Transfer & data_in latched $\rightarrow$ DIR \\
& & DOR $\rightarrow$ DOR $R_{\text {shadow }}$ \\
& & address latched $_{\text {local memory address }}$ \\
2 & Memory & read access $:$ memory data $\rightarrow$ DOR \\
& Access & write access $:$ DIR $\rightarrow$ memory data \\
& Return & DOR $\rightarrow$ data_out \\
& \& Restore & DI $R_{\text {shadow } \rightarrow \text { DIR }}$ \\
& & DOR $R_{\text {shadow } \rightarrow \text { DOR }}$ \\
& set ready flag \\
\hline
\end{tabular}

Table 5.2: Interrupt Cycles

essential to directly connect CRYPTONITE to the PCI bus or similar bus interfaces are not covered by this work.

\subsection{The Control Unit}

Architecturally, CRYPTONITE consists of several independent units being controlled by a central dispatcher which generates the appropriate control signals based on internal state and current instruction word.

This dispatcher is called the Control Unit (CU) and is depicted in Figure 5.3. It contains the instruction word decoder and additional units which are needed for proper program processing; it supports linear addressing and conditional branching as needed for conditional constructs like IF / THEN and loops. It also provides immediate values being encoded into the command stream. This command stream is fetched by the CU from program memory.

\subsubsection{Supplying immediate values}

The CRYPTONITE architecture supports two types of immediate values: Big immediates (64 bit) for initialization of ALU and MU registers plus 12bit address/immediate values used for counter register initialization (8-bit) 


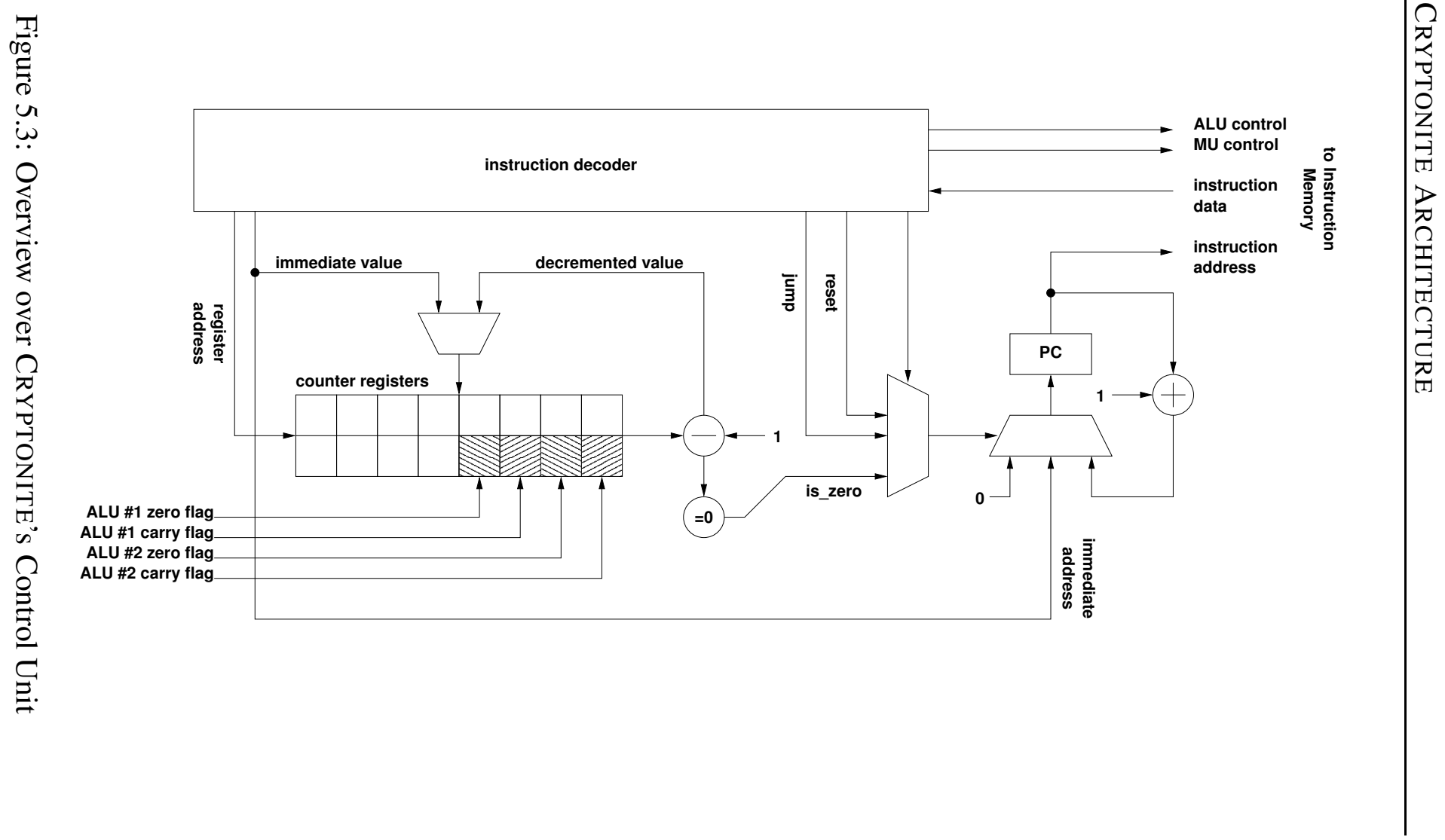


and providing branch addresses for conditional and unconditional branches (12-bit). The 64-bit immediates are named global immediate values (GIV) since they are common for all addressable registers within a so-called strand consisting of ALU, MU and associated memory, where the 12-bit value can be used within the Control Unit and for register initialization within the MAU.

Since initialization with GIVs usually does not take place very often and happens only during an algorithm's initialization stage a special immediateload operation LDI changes the interpretation of the VLIW-style instruction word as explained in Appendix B. During an LDI cycle both strands are put in a special load mode and normal operation of ALU and MU is disabled for this cycle.

This decision was made to allow the load of registers with values of up to 64-bit in size while not dramatically the size of the already wide instruction word. This load mechanism fits into the already existing architecture. For this reason, GIVs are internally treated as values being read from data memory.

\subsubsection{Counters, looping, and conditional branching}

The CRYPTONITE architecture only supports basic down-counting loops based on 12 counter registers $c r 0$ to $c r 11$ where each of these registers can be loaded with an immediate value for loop initialization. As implemented in the architecture, loops are pre-decrement which means a register's value is read out, decremented and based on the result a branch is taken or not. In terms of functionality it is similar to the DJNZ instruction as known from several processors like the Intel x86 processor family [34] or Zilog's Z80 [137].

While this minimal loop support is sufficient for almost all analyzed algorithms, the IDEA algorithm also needs conditional branching based on ALU results. To make this concept fit into the existing ALU and CU designs, the counter registers $\operatorname{cr} 12$ to $\mathrm{cr} 15$ will sample the current state of the two ALU's zero and sign outputs and serve as flags rather than being used as ordinary counter registers. This way the flag states can be checked by the equal-to-zero comparator as already used together with counter registers $\operatorname{cr} 0$ to $\operatorname{cr} 11$. 


\subsubsection{System Control}

As mentioned in Section 5.6, the Control Unit generates the necessary control signals from the current instruction word for parametrization of all onchip units.

The instruction word has 10 parts and contains the coded control words for the five units which are Control Unit, two ALUs, and two Memory Units plus the address/immediate field. A description of the instruction word format is given in Appendix B.

\subsection{The Arithmetic Logical Unit}

Crypto algorithms usually employ a variety of basic arithmetic operations like Boolean operations, addition/subtraction, and bit-shifting or -rotation. Depending on the algorithm quite a number of intermediate results have to be stored which ideally can be held in registers rather than external memory to avoid latencies caused by external memory access.

However, a register file must not exceed a specific size to follow the technological specifications. Where a huge and massively interconnected register file, i.e. having many input and output ports, will certainly ease program realization it will easily exceed technology limitations.

In this chapter an arithmetic logical unit (ALU) will be described which is not only suited for fast and efficient computation of typical crypto algorithms and but will also fulfill the technological requirements as listed in Section 5.1.

\subsubsection{Overview}

CRYPTONITE's ALU consists of 3 functional units which are the Register File (RF), the Arithmetic Unit (AU), and the XOR Unit (XU) as depicted in Figure 5.4. The following sections will discuss these units in detail.

\subsubsection{The Register File}

The RF holds four 64-bit registers, $r 0$ to $r 3$, which can be individually loaded from two internal buses called $\mathrm{A}$ and $\mathrm{X}$. The $\mathrm{A}$ bus transports the 


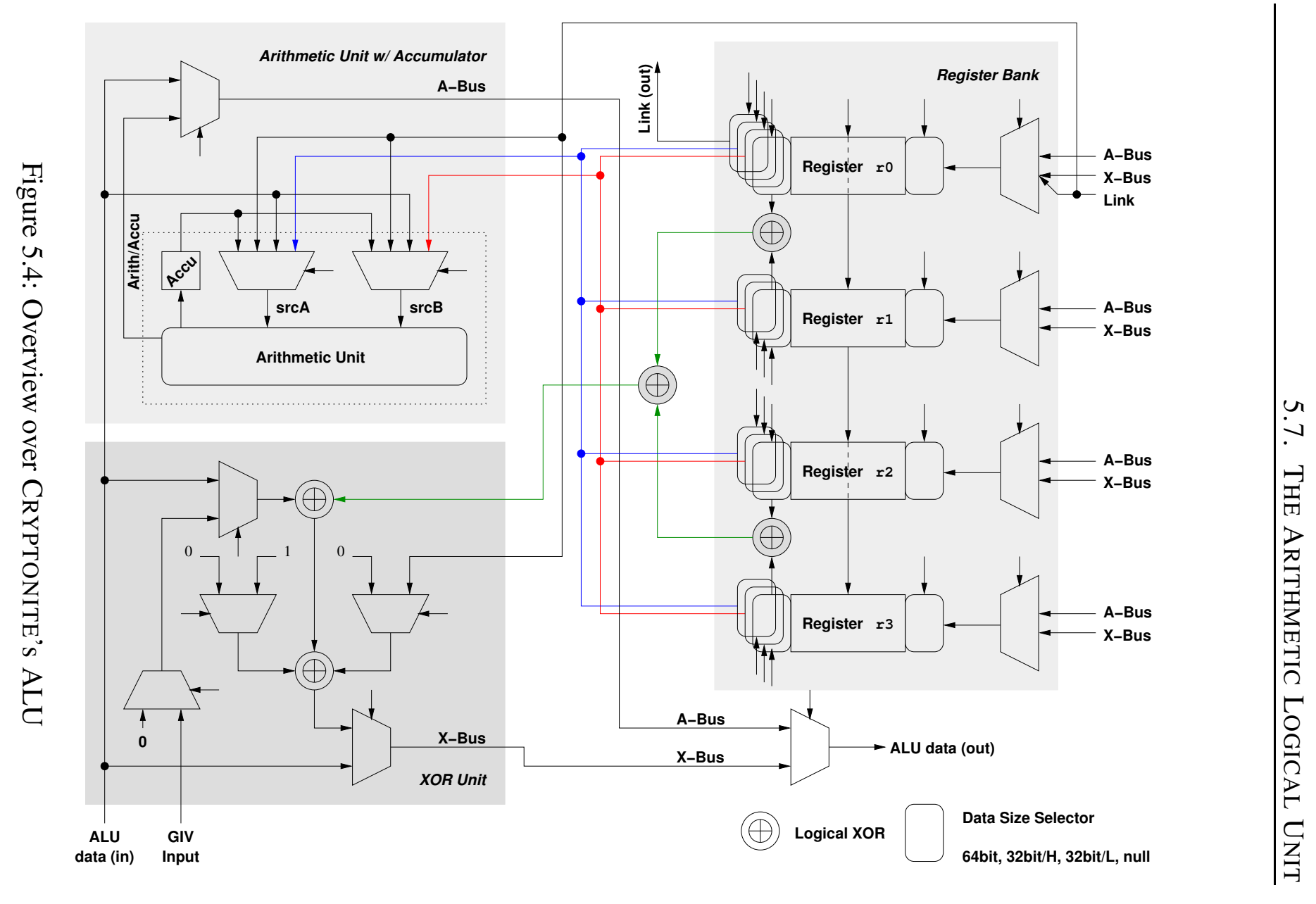


result of a previous arithmetic operation where the $X$ bus supplies the result of a previous XOR operation. For exchange of data between the two ALUs, register $r 0$ of each ALU register file can also be loaded from the ALU interlink bus.

Once a bus is assigned to a register the input data size selector determines whether the complete 64-bit data, or the high or low 32-bit portion should be loaded into the register. Alternatively, the data size selector can be used to simply apply a zero value for clearing the register as already mentioned in Section 4.2.1.

At the output port of the register a similar data size selector is employed which again enables selection of the entire 64-bit, or high or low 32-bit of a stored value, or to "mute" the register by outputting a zero value without changing the register's content. Each register has three output ports for addressing the XOR unit and the source inputs \#1 and \#2 of the ALU. There is no immediate way of storing a register value to memory (or the data input register, respectively). This is only possible for results of arithmetic or boolean operations. In case the unaltered register value needs to be stored, the used AU or XOR unit has to perform a NOP operation. Direct read-out of register values was omitted since this would need another output unit per register and four additional 64-bit datapaths within the chip.

Again, register $r 0$ can serve for interlink purposes and has a fourth output port for feeding the AU interlink.

\subsubsection{The Arithmetic Unit (AU)}

The AU is able to perform all standard arithmetic and Boolean operations plus specific functions needed to improve speed on AES/Rijndael calculation based on input values provided through the Register File registers, the associated Memory Unit's Data Output Register, and the AU's internal accumulator. The accumulator can be used for storage of intermediate results and can be loaded from either of the two source inputs. Its use is optional.

Besides basic arithmetic (addition \& subtraction) and Boolean functions (AND, OR \& XOR) the ALU employs a barrel shifter which can be used to shift or rotate either a 64-bit value or two 32-bit values by an arbitrary number of bits and enables special functions as explained in Section 5.7.3.1. The AU also offers a swap instruction by which the 32-bit halves of a 64bit quantity can be swapped; as a result of this operation and the presence 
of a barrel shifter, also combined swrt operations based on swapping and left-rotation are possible which are employed for fast AES/Rijndael implementation [45].

The analysis of IDEA and RC6 motivated the use of a parametrizable multiplier. For example, IDEA uses 16-bit*16-bit $\bmod _{16}$ multiplications, RC6 - depending on its configuration - employs 32-bit*32-bit $\bmod _{32}$ to 64bit*64-bit $\bmod _{64}$. Such a unit would greatly enhance the performance of these algorithms and also support asymmetric cryptography. For the CRYPTONITE architecture, this unit is optional because of the lesser relevance of IDEA and RC6 and since its focus is symmetric If implemented, only the modulo operation should be configurable with respect to the 64-bit architecture. The input data size is always 64-bit as with all other ALU units.

The result of an arithmetic operation is put on the internal A bus and can be routed to any of the four registers or the Data Input or Ouput Register of the associated Memory Unit.

\subsubsection{Special Functions}

As a result of the AES/Rijndael analysis a set of instructions was developed to speed up that algorithm. However, unlike the DES Unit which is only applicable to (3)DES, these instructions are not AES/Rijndael-specific and can be used for other algorithms as well.

These functions sometimes operate in 32-bit quantities. In such cases, indices $h$ and $l$ denote the referring 32-bit portion of a 64-bit quantity with $h$ being the leftmost and $l$ the rightmost one. Numeric indices refer to the corresponding byte (8-bit portion) of a 64-bit quantity with byte \#0 being the rightmost one.

- The upper64 operation takes two 64-bit quantities $x$ and $y$ and outputs the 64-bit quantity $x_{7} x_{3} y_{7} y_{3} x_{6} x_{2} y_{6} y_{2}$ where the indices denote the referring byte within the input values.

- Similarly, lower64 generates the 64-bit quantity $x_{5} x_{1} y_{5} y_{1} x_{4} x_{0} y_{4} y_{0}$.

$$
\begin{array}{ll}
\text { upper64 } & f(x, y)=x_{7} x_{3} y_{7} y_{3} x_{6} x_{2} y_{6} y_{2} \\
\text { lower64 } & f(x, y)=x_{5} x_{1} y_{5} y_{1} x_{4} x_{0} y_{4} y_{0} \\
\hline
\end{array}
$$

Table 5.3: The upper64 and lower64 functions 


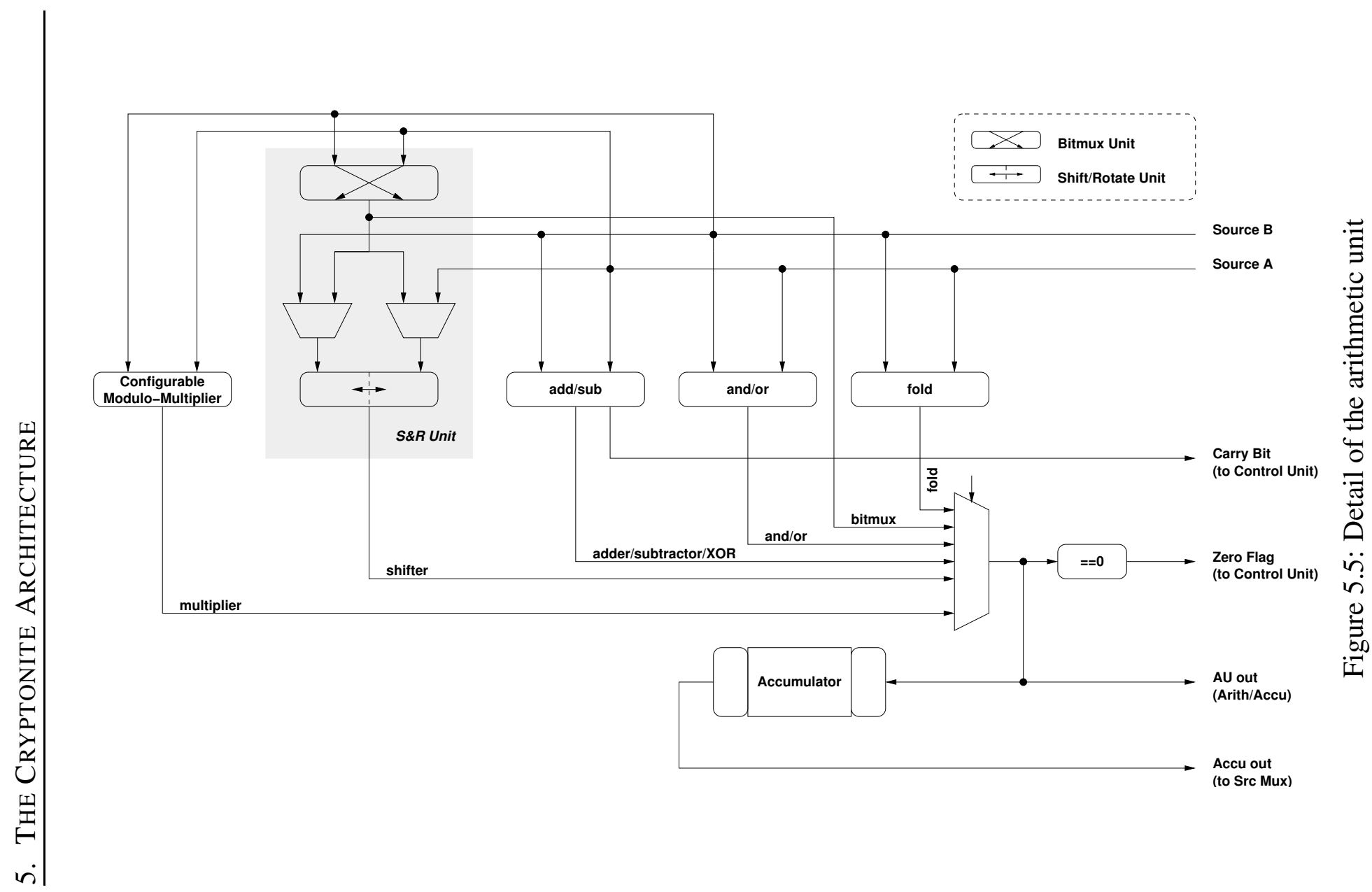


- The swap instruction takes two 64-bit quantities $x$ and $y$ and generates the 64-bit result $x_{l} y_{h}$ (or $x_{h} y_{l}$ depending on the parametrization of the register output multiplexers). The indices denote the referring 32-bit half of an input value.

- The swrt instructions are based on the swap instruction and rotate operations; they take a two 32-bit quantities like the swap instruction but rotate these by individual amounts as shown in Table 5.4.

\begin{tabular}{|ll|}
\hline swap & $f(x, y)=x_{l} y_{h}$ \\
swrt0 & $f(x)=\left(x_{h} \lll 8\right) y_{l}$ \\
swrt1 & $f(x)=\left(x_{h} \lll 24\right)\left(y_{h} \lll 16\right)$ \\
\hline
\end{tabular}

Table 5.4: The swap functions

- A set of fold instructions is included. These perform a series of XOR operations based on two input values and intermediate results as shown in Table 5.5.

\begin{tabular}{|ll} 
foldb32 & $f(x, y)=\forall 0 \leq i \leq 3:\left\{\begin{array}{l}i=0: x_{0} \oplus y_{0} \\
i \neq 0: f_{i-1} \oplus y_{i}\end{array}\right.$ \\
foldb64 & $f(x, y)=\forall 0 \leq i \leq 7:\left\{\begin{array}{l}i=0: x_{0} \oplus y_{0} \\
i \neq 0: f_{i-1} \oplus y_{i}\end{array}\right.$ \\
foldw64 & $f(x, y)=\forall 0 \leq i \leq 1:\left\{\begin{array}{l}i=0: f_{h}=x_{l} \oplus y_{h} \\
i=1: f_{l}=f_{l} \oplus y_{l}\end{array}\right.$
\end{tabular}

Table 5.5: The fold operations

Within the AU most of these functions are performed by the so-called bitmux unit which performs the mentioned swap, upper64 and lower64 operations as shown in Figure 5.5. This unit also feeds the barrel shifter to allow complex swap and rotate operations. The fold operations are computed in a separate fold unit. 


\subsubsection{The XOR Unit}

The exclusive-or (XOR) operation is the key operation of all cryptological algorithms which makes it vital to implement a fast and efficient method for performing a series of XOR operations.

In CRYPTONITE the XOR Unit not only performs the XOR operation on two to six operands consisting of the register file together with the content of the associated Memory Unit's data output register dor and an optional sixth value provided through the ALU interlink as described in Section 5.7.5, but also allows the negation of a bit pattern. For that reason the XOR unit has an internal switch to optionally invert the result of an XOR operation.

To minimize on-chip buses the XOR unit is partly embedded into the data path. The first XOR stage already sits within the register file and combines the values of register 1 and 2, or 3 and 4 respectively. The result of this first XOR stage is then combined in another stage before it is fed into the XOR unit's main part as depicted in Figure 5.4. This method of embedding an arithmetic function into the physical on-chip data path is currently filed for patenting.

Like register $r 0$ of the Register File also the XOR Unit has a special interlink input enabling to forward the value of the opposite ALU's register ro directly into the XOR Unit's main part to be concatenated with the result of the register combination if needed. Finally, the overall result can be inverted and the result is put onto the ALU's X bus to be routed to any of the four registers $r 0$ to $r 3$ or the associated Memory Unit's data input dir and data output registers dor. The XOR unit furthermore can distribute a GIV (provided by the $\mathrm{CU}$ as mentioned in 5.6.1) to the aforementioned registers.

\subsubsection{Avoiding register pressure: Crosslinking the arith- metic units}

The original design considered an 8-register file with full connectivity of registers to both ALUs. This concept was cancelled for speed reasons since full connectivity would mean more read and write ports per register and less optimal routing, especially long wires.

Instead, the concept of individual register files per ALU was taken with only four registers per file. This turned out to be enough for most cases; however, it limited the possibilities of parallel computation of independent 
values since the computation of a single formula had to be fully assigned to one ALU. Also, some algorithms turned out to lay rather high pressure on the register file during a sequential calculation forcing the writeback and reload of intermediate values to and from memory.

For this reason an interlink mechanism was designed to allow forwarding of register contents to the opposite ALU. To minimize technological impact, mainly speed and delay issues, link exchange takes only place between register $r 0$ of each ALU. To allow immediate use of these values within ongoing computations, it is additionally possible to directly feed the current value of register $r 0$ into the opposite ALU's arithmetic and XOR units. Doing so avoids a one-cycle delay otherwise occuring for value transfer from one register $r 0$ to its opposite ALU's sibling.

\subsection{The Memory Unit}

Each ALU has a corresponding Memory Unit (MU) for data storage. The MU consists of an address generation unit (AGU) responsible for proper address generation based on a selected addressing scheme (see Section 5.8.1), and a Data Input/Output Unit (DIO) (see Section 5.8.3) which is basically used for data transport but also contains the DES Unit as explained in Section 5.8.4. Attached to the MU is the local data memory as depicted in Figure 5.1.

\subsubsection{Address Generation Unit}

The AGU as depicted in Figure 5.6 is responsible for generating addresses during data memory access and inherently supports direct and S-Box addressing; indexed and modulo addressing are also supported by the AGU, but computation of addresses takes one cycle. When using these addressing modes the programmer must remember this post-increment/post-modulo model (as opposed to pre-decrement as used within the control unit for loop support). The complete list of supported addressing modes is listed in Table 5.6.

To enable external access to data memory, it is also possible to supply an external address instead of the generated one. This allows an external processor, a network protocol processor for example, to directly read computed 
results or feed in new data like plain-text chunks or key updates; similarly, look-up tables can be easily exchanged using an external processor.

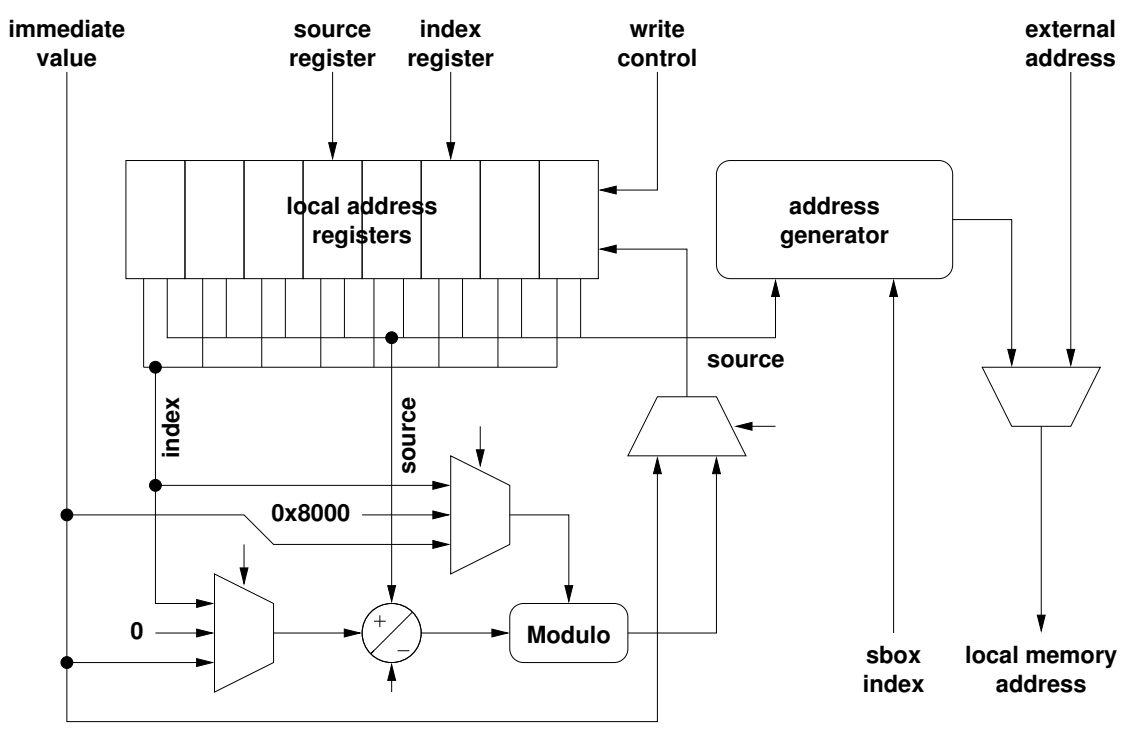

Figure 5.6: The Address Generation Unit

The basis of all address generation is the Local Address Registers (LAR). In the simplest configuration, direct addressing, a LAR holds an address pointer which is directly used as a memory address; indexed addressing is supported in two ways, using immediate index values as supplied by the control unit (which means that the index value is embedded into the opcode) or index values derived from other LARs. With both methods, the index value is added to an address supplied by a LAR. Also, as mentioned before these modes provide deferred results. The reason for doing so was to keep the memory address generation as fast as possible to be able to provide memory data right in time and minimizing the address setup time for the on-chip memory.

With these three modes the AGU also supports modulo addressing to automatically keep a generated address within a dedicated memory segment. This is especially useful for hash algorithms like MD5 or SHA-1 where a table of 16 (20 for SHA-1) entries is accessed using indexed addressing with an offset pointing into the table and an index increment greater than one. Figure 5.8 illustrates the effect of modulo addressing applied to indexed 


\begin{tabular}{|l|l|c|}
\hline \multicolumn{1}{|c|}{ Addressing Mode } & Address Computation & \multicolumn{1}{c|}{ LAR Update } \\
\hline \hline direct & $a d d r=L A R$ & \\
,,$w /$ register modulo & $a d d r=L A R_{x}$ & $L A R_{x}=L A R_{x} \% L A R_{y}$ \\
”,w/ immediate modulo & $a d d r=L A R_{x}$ & $L A R_{x}=L A R_{x} \% i d x$ \\
S-Box & $\forall 0 \leq i \leq 7: a d d r_{i}=(L A R \wedge 0 \mathrm{x} 7 \mathrm{f} 00) \vee i d x_{i}$ (LAR unchanged) \\
immediate-indexed & $a d d r=L A R$ & $L A R=L A R+i d x$ \\
ditto, w/ register modulo & $a d d r=L A R_{x}$ & $L A R_{x}=\left(L A R_{x}+i d x\right) \% L A R_{y}$ \\
register-indexed & $a d d r=L A R_{x}$ & $L A R_{x}=L A R_{x}+L A R_{y}$ \\
ditto, w/ immediate modulo & $a d d r=L A R_{x}$ & $L A R_{x}=\left(L A R_{x}+L A R_{y}\right) \% i d x$ \\
\hline
\end{tabular}

Addressing modes written in italics are based on architectural side-effects

and have not been designed in by purpose.

Table 5.6: Addressing modes supported by CRYPTONITE's AGU 
addressing: The dashed location would be addressed with straight index addressing; using modulo- 4 addressing, however, the resulting address is location 1 instead of 5 .

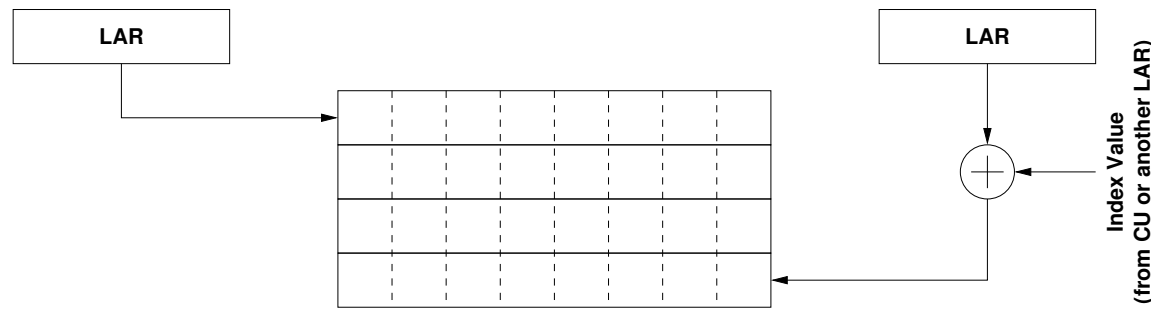

Figure 5.7: Direct and indexed address generation

Like the indexed modes also the modulo operation is deferred. For hardware reasons the modulo operation is limited to powers of two which makes it possible to implement this function using the Boolean AND operation as opposed to an extensive integer division unit.

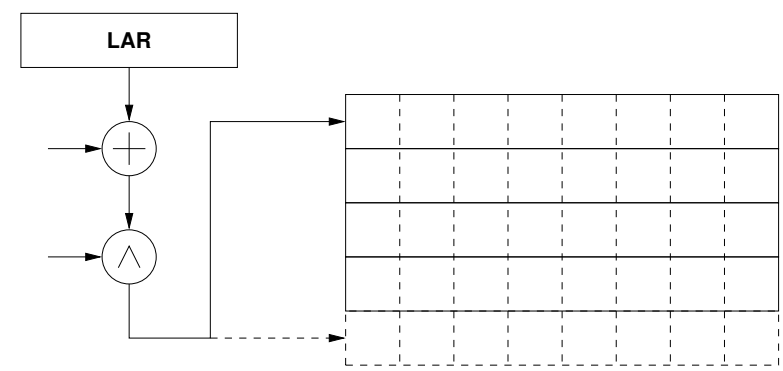

Figure 5.8: Modulo addressing

\subsubsection{Speeding up table lookups: S-Box support}

A very special feature of the AGU is the S-Box addressing mode. Unlike the previously described modes which address the memory in 64-bit mode, SBox addressing makes use of the memory's feature to be addressed as eight 8-bit quantities instead of a single 64-bit chunk. The use of 64-bit memory 
as eight user-definable 8-bit S-Boxes is a unique feature not found in crypto processor architecture so far and is also currently filed for patenting.

The address per 8-bit chunk is derived from an LAR supplying the SBox address plus the S-Box index. Since each index is 8 bits in size the address space of an S-Box is 256 addresses.

With S-Box addressing mode, the base LAR provides the S-Box number where the index value contains the eight 8-bit indices into the selected SBox as depicted in Figure 5.9. S-Box access can happen only aligned to 2048-byte boundaries ( 256 addresses times 8 bytes); unaligned addressing is not possible since the lower address bits of the LAR are replaced by the corresponding S-Box index. This method was chosen over the use of an adder for timing reasons to not increase latency of this critical data path.

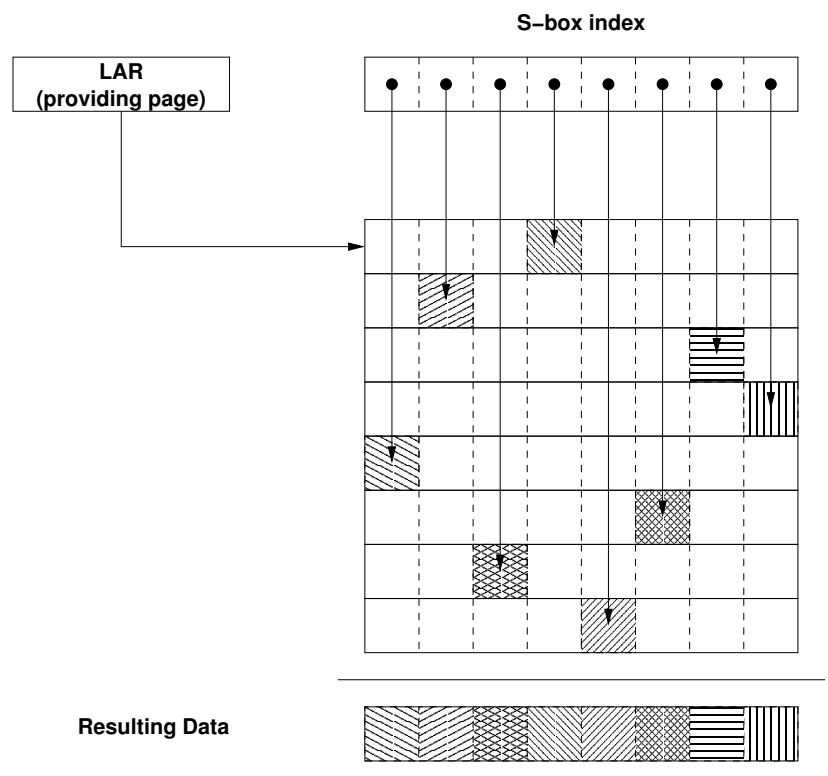

Figure 5.9: S-Box address generation

\subsubsection{The Data Input/Output Unit}

Due to the very tight timing of the architecture it is necessary to provide a method of synchronizing data for long distance transport as needed for transfer of data between embedded SRAM and the ALUs. 
The DIO as depicted in Figure 5.10 consists of a Data Input Register (DIR) where data coming from the ALU is written into prior to be stored to memory. Respectively, a Data Output Register (DOR) exists which will buffer a value transferred from memory to ALU. Since the DIR will also provide the S-Box index coming either from ALU or a previous DES round, the DIR input can select either ALU data or a result coming from the DES unit discussed in Section 5.8.4. As already mentioned in Section 5.7, the DOR can serve as an additional source or destination for arithmetic operations. In Section 5.5 it has already been pointed out that DIR and DOR have accompanying shadow registers used to rescue and restore the original register values during external access interrupt handling.

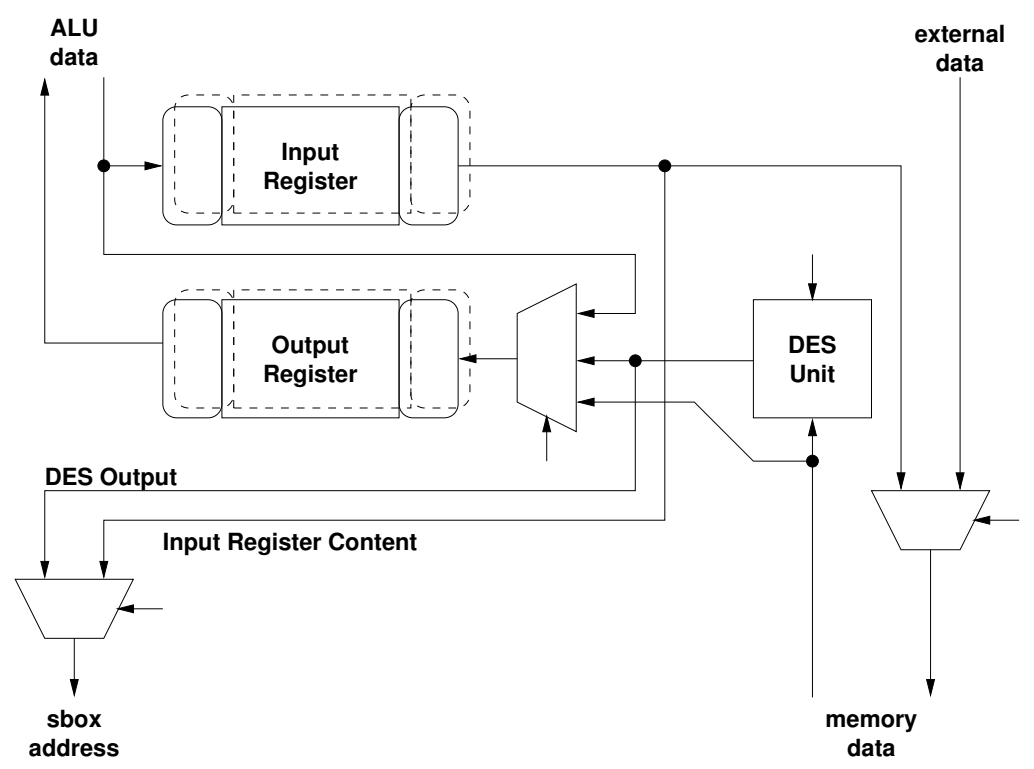

Figure 5.10: Data I/O from embedded SRAM

Besides these registers, the DIO also contains a DES Unit which will provide the necessary functionality needed for fast and efficient (3)DES computation. To allow further processing or storage of a DES operation's result, these can be either directly fed back to memory or into the ALU through the output register. Since DES provides independently computed S-Box indices, it can directly access the S-Box address path providing the 
necessary 64-bit S-Box index value. The following Section 5.8.4 will discuss the DES Unit in detail.

\subsubsection{The DES-specific unit}

The heart of the DES algorithm is the non-linear function based on the socalled S-Box table lookup. All other functions within the DES algorithm are mainly bit-shuffling plus shifting of the two key halves.

These bit-shuffling functions are DES-specific and are not reusable for other functions. Similarly, the 28-bit rotating shift operation does not apply to other cryptographic functions which usually operate on multiples of 8-bit, mainly 32-bit. For this reason and since the main cryptographic operation of DES is an S-Box lookup the DES-specific functions were transferred from ALU into the memory unit. Doing so, the ALU can be less complex; in addition, the necessary physical data paths for DES computation are kept as short as possible which potentially supports higher clock frequencies.

\subsubsection{ALU-based initial approach}

The initial approach was to implement the bit-shuffling functions into the ALU and allow another shifter configuration for the $2 x 28$ bit operation. The idea behind this approach was to avoid a huge, monolithic function for the sake of an increased cycle count.

- DES input permutation

- DES data expansion

- DES key compression

- DES P-Box permutation

- DES final permutation

- 2x28-bit rotation configuration for barrel shifter

Table 5.7: Special functions for non-monolithic DES implementation 
As already explaind in Section 3.3.2 this leads to 5 cycles per DES round based on the 5 DES-specific ALU functions. In addition, a special shifter configuration for DES key computation is needed resulting in special operations as listed in Table 5.7.

\subsubsection{Speed-up by pipelining DES}

Looking at DES at a more coarse-grained scale, it turns out that DES is easy to pipeline: All specific functions apart from input and final permutation can be melted into two monolithic functions which as listed in Table 5.8. Besides these two functions only input and output permutation apply which can be realized as translation functions embedded into the data path of the $L$ and $R$ variables during computation as shown in Figure 5.11.

However, these monolithic functions are clearly DES-specific and can certainly not be reused for anything else. It would not be sensible to bloat the ALU with this heavy-weight functional unit as shown in Figure 5.11, especially since both functions are tighly coupled to memory lookups which makes the memory unit a by far more reasonable place to integrate this unit into.

Separating the DES unit also means that it needs to contain the necessary control logic for providing the round constants (shifting amount) since these values cannot be externally applied anymore.

For this reason, a clearable 4-bit register with an associated incrementer is employed for address generation. This address is fed into a small round constant memory which contains of 16 entries of 2 bits each to provide a single round's shift amount. The address can be inverted resulting in a backward address count as needed for decryption.

- expand $R_{i-1}$, shift \& compress key, and XOR the results

- permutate the S-Box result using P-Box shuffling and XOR this result with $L_{i-1}$; forward $R_{i-1}$ to $L_{i}$

Table 5.8: Special functions for pipelined DES implementation 


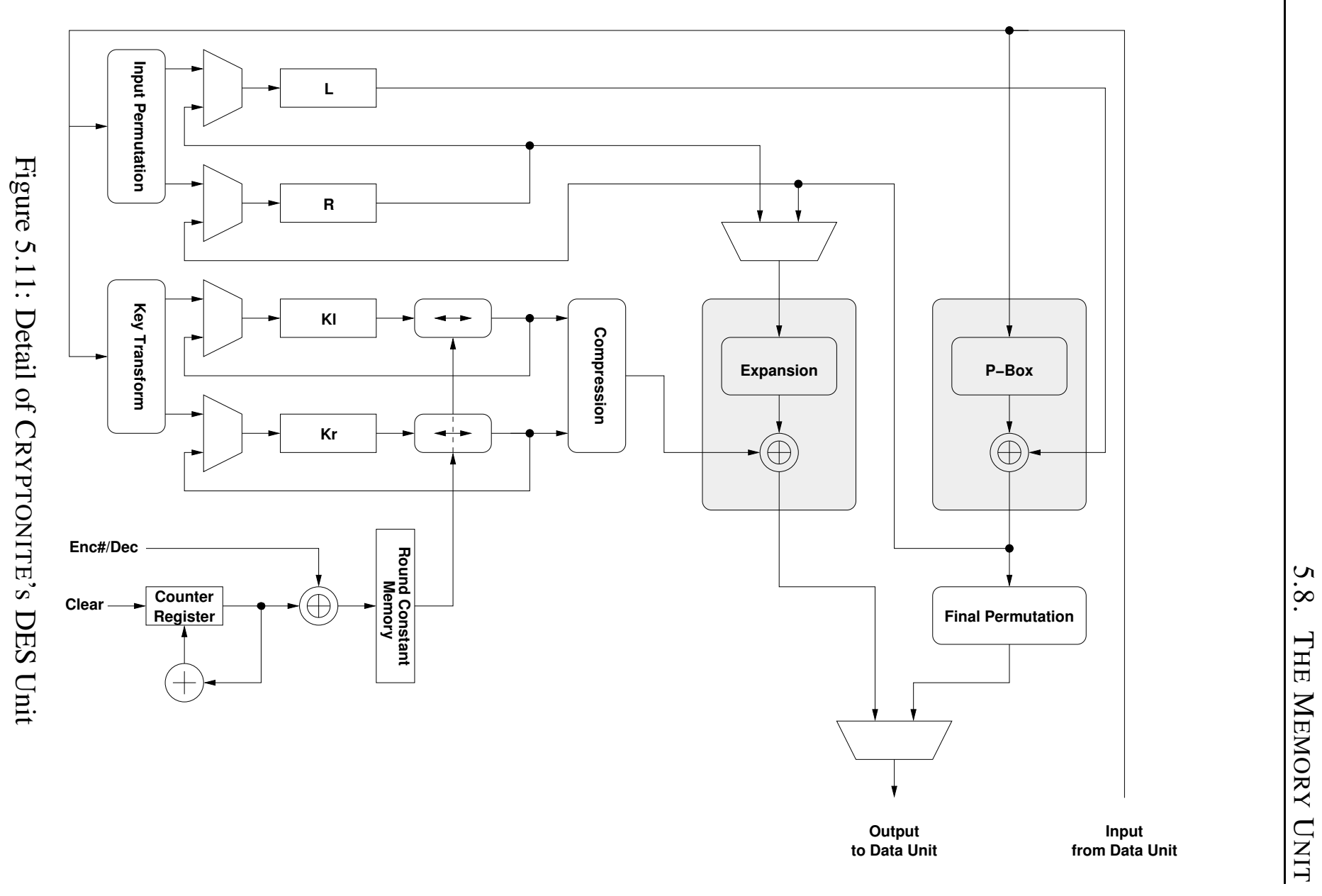




\subsection{Summary}

In this chapter, the architecture of the proposed CRYPTONITE architecture was presented and reasons were given why such a specialized architecture makes sense even with powerful modern general purpose processors being able to deliver similar computation power.

This architecture consists of a Control Unit (CU) parsing the instruction stream and creating control signals for the other units. These other units form two strands consisting of Arithmetic Logical Unit (ALU) and its corresponding memory unit (MU) and associated SRAM-type data memory. Besides, the $\mathrm{CU}$ is responsible for data transfer from program to data memory and vice versa. It also controls program flow: For this reason it employs counter registers, status flags and a small decrement/compare logic to allow conditional branching.

The ALU further divides into an Arithmetic Unit (ALU) including the accumulator, the XOR Unit (XU) and a register file (RF) holding four 64bit general purpose registers. Memory access uses two buffering registers named Data Input (DIR, for memory writes) and Data Output Register (DOR, for memory reads). Computation results can be stored in either DIR, DOR or both, where the DOR can be read back by AU and XU through an individual input. Storage of RF register values to memory are only possibly through the DIR. The ALU employs a crosslink mechanism which allows forwarding either register $r 0$ or an ALU result to the sibling ALU.

Similarly, also the MU dissects into further units which are Address Generation Unit (AGU) and Data I/O Unit (DIO). The AGU holds two sets of eight local address registers; these sets are individual for read and write operations. It contains simple logic to support various addressing modes as explained in Section 5.8.1, S-Box addressing being one of these allowing 8 parallel S-Box lookups within one cycle. A DES-specific unit is part of the DIO.

The presented architecture is the result of the algorithm analysis presented in Chapter 3. It is as general as possible and contains only special functional units and operations where needed for speeding up certain algorithm computation.

Based on the algorithm analysis in Chapter 3 this architecture provides the necessary computation power to allow fast and efficient implementation of several crypto algorithms, but was kept as simple as possible to enable 
high clock rates when being implemented in hardware. The performance numbers of both, algorithm implementations and hardware realization, will be presented in Chapter 6 . 
5. The Cryptonite ArChitecture

92 


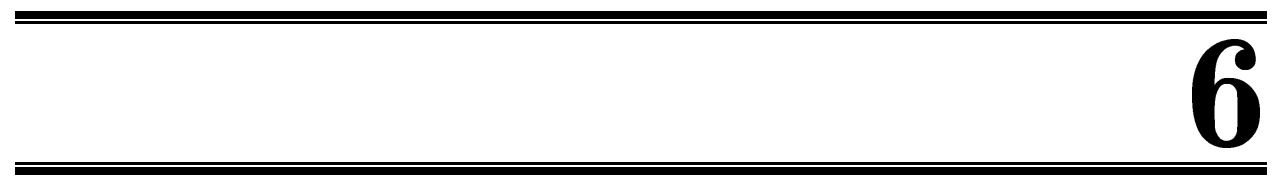

\section{Implementational Aspects}

The previous chapters focused on algorithm analysis (Chapter 3), how these algorithms influenced the CRYPTONITE architecture (Chapter 4), and finally presented the resulting architecture (Chapter 5). This chapter now will present actual results based on the proposed architecture to answer how the algorithms will perform on CRYPTONITE and what the technology implications are.

Since no complete CRYPTONITE processor has currently been built, the numbers presented for CRYPTONITE are based on software simulation and synthesis results of dedicated hardware parts. Although the author is confident that these simulation results accurately reflect reality, he wants the reader to be clear about the numbers not being based on measurements performed on existing hardware.

\subsection{Software Implementation}

This section concentrates on the algorithm implementations in software based on the architecture described in Chapter 5 to provide comparative numbers for processing speed and data throughput. To collect these numbers, test implementations in of each algorithm were created. These implementations, with the exception of AES, were run on a self-written simulator to verify the correctness of these implementations. This simulator is basically a $\mathrm{C}$ library emulating the CRYPTONITE assembly language as described in Appendix $\mathrm{C}$ by providing appropriate function calls together with sanity checks to ensure that an algorithm implementation does not vi- 
olate CRYPTONITE hardware contraints. AES was implemented using the Standard Modelling Language (SML) language and runtime environment. Both simulation environments do not provide any timing analysis besides cycle count; hardware parameters will be investigated in Section 6.2 using Synopsys [118] hardware development software.

Cycle count of all algorithms implemented using the aforementioned softwre simulators is summed up in Table 6.1. Comments to each implementation are given within the referring subsection. Where appropriate, cycle count is provided as a formula $s+r * c+p$. Here, $s$ represents number of cycles used for setup, $r * c$ gives number of rounds times number of cycles per round, and $p$ represents cycles used for clean-up and post-processing.

\begin{tabular}{|l|r|cc|}
\hline \multirow{2}{*}{ Algorithm } & Cycle Count & Throughput (@ 400MHz) \\
\hline \hline AES-128/128 $\square$ & $2+9 * 8+6=80$ & 1.6 & 640 \\
AES-128/128 $\square$ & $2+9 * 7+5=70$ & 1.83 & 732 \\
DES & $4+15^{*} 2+1=35$ & 1.83 & 732 \\
3DES & $3^{*}(30+5)=105$ & 0.61 & 244 \\
IDEA & $8^{*} 11+2=90$ & 0.71 & 284 \\
RC6 & $3+20 *(7+3)+3=206$ & 0.62 & 249 \\
\hline MD5 & $2+16^{*}(8+8+7+8)+6=504$ & 1.02 & 406 \\
SHA-1 & $2+16^{*}(8+7+8+7)+6=488$ & 1.05 & 420 \\
\hline
\end{tabular}

Table 6.1: CRYPTONITE Performance Data for Selected Algorithms

\subsubsection{AES/Rijndael}

For comparison, the 128/128-configuration of AES/Rijndael [45] was implemented in two ways. The simpler implementation $(\square)$ relies on precomputed round keys, the second implementation $(\otimes)$ creates needed round keys on the fly from the supplied user key.

Cycle count sums up to two initial cycles, 8 cycles $(7$ cycles for the simple version) for the first 9 rounds and 6 (5) cycles for the last round and writeback. Performance numbers are given for both versions. 


\subsubsection{DES and 3DES}

These implementations are fairly trivial since DES is supported through special instructions. Since these instructions are strictly sequential and only one key register exists, it does not make any difference if 3DES is used in twoor three-key configuration since the key register needs to be reloaded for every key change.

The given formula for cycle count represents a 4 cycle init phase followed by 16 rounds per 2 cycles each and a final cycle for writeback.

\subsubsection{IDEA}

The IDEA implementation does only include the plain encryption/decryption algorithm; key generation is omitted since it needs division and modulo arithmetics which are not included within the CRYPTONITE architecture. Furthermore, it makes the assumption, that a $\bmod _{16} 16$-bit multiplier is present.

For these reasons, the cycle count cuts down to 8 rounds per 11 cycles plus two cycles for final processing and result writeback.

\subsubsection{RC6}

RC6 in AES configuration showed an interesting result; although this algorithm configuration seems to be perfectly suited for the CRYPTONITE architecture it needs 11 cycles ( 8 for computation, 3 for swap) per round. This implementation partly omits the round key generation, which means that the implementation assumes that the key array has already been written to memory. Also, it makes the assumption that a $\bmod _{32}$ 32-bit multiplier is present.

Then, the cycle count distributes on 3 cycles each for init and postprocessing plus 220 cycles for the 20 rounds.

\subsubsection{MD5 and SHA-1}

Due to the similarities of the two algorithms their implementations are fairly identical. SHA-1 shows slightly better performance since it incorporates two "fast" rounds of 7 cycles as opposed to only one for MD5. 
Initialization takes 2 cycles, postprocessing 6 cycles. The main impact comes from the 16 iterations per each of the 4 rounds and equals 480 (SHA1) or 486 (MD5) cycles.

\subsection{Hardware Implementation}

Within this section, the hardware parameters are evaluated to generate realistic numbers for core speed and die or logic size. These numbers can be compared against parameters provided by core vendors; however, these are highly dependent on the technology used. Usually, the numbers are based on FPGA families like Altera APEX [8, 7] or Xilinx Virtex [132, 133] and ASIC fabrication processes from TSMC [119] and UMC [125]. Although they lose relevance when considering chip mask design, the numbers still give an estimation about the proposed $400 \mathrm{MHz}$ core speed being possible or not.

For testing and implementing the VHDL models as well as creating timing and chip usage reports the Synopsys software package running under Sparc/Solaris together with the FPGA compiler provided by Xilinx [65] was used.

\subsubsection{Technology Requirements}

The CRYPTONITE architecture consists of the processing unit itself and associated memory which means that the used fabrication process must be able to provide fast and efficient static memory (SRAM). This SRAM has to be fast enough to deliver data content within the running cycle.

As for memory size, the proposed address space requires 12 address lines. With these, $4 \mathrm{kBit}^{*} 64$ equivalent to $32 \mathrm{kB}$ of plain data or 16 sets of $8 \mathrm{~S}$-Boxes can be held in memory which the author believes to be adequate for future algorithms.

When realized on FPGAs and ASICs, a dedicated hardware multiplier would help greatly. Otherwise, the multiplier has to be realized using standard techniques which are quite space inefficient and create long delays. 


\subsubsection{Register File}

The register file consists of four identical registers with an input and output multiplexer. These multiplexers make possible to read in data from either A or $\mathrm{X}$ bus, for register $r 0$ also the interlink serves as an additional input.

\subsubsection{Arithmetic Unit: Shift \& Rotate Unit}

This is the most complex unit within the arithmetic unit consisting of the swap multiplexer, a barrel shifter and the final bit splicer containing the longest single data path.

\subsubsection{Address Generation Unit}

The AGU is the by far most timing critical unit. It needs to provide the memory address fast enough to comply with the memory's setup time allowing single-cycle memory accesses regardless of selected addressing mode.

\subsubsection{Implementation Results}

For test purposes Xilinx' [132] latest FPGA family Virtex-II Pro [133] was chosen since this family is almost the biggest FPGA family on the market. The reason doing so was to avoid running into resource limitations like too few logic elements, interconnects or I/O pins when synthesizing and analyzing parts of the CRYPTONITE architecture. However, it appeared that even with the largest model the sheer number of employed signals made it impossible to test specific behaviour like the ALU interlink timing for instance. For this, two complete ALUs had to be instantiated with a rather large amount of input and output signals. Interestingly, this problem would not arise for testing the complete design, since these numerous signals would not occur as physical I/O signals on the final design but only as internal signal nodes.

For this reason, only small parts of the CRYPTONITE architecture could be synthesized and analyzed to get an idea about the signal delays within the chip.

These results were very promising: Without further optimization the designs reach speeds up to $141 \mathrm{MHz}$; the limiting factor here is neither logic nor routing, but delay caused by routing the signals to the $\mathrm{I} / \mathrm{O}$ pads: 


\begin{tabular}{|l|rr|rrrr|}
\hline & \multicolumn{2}{|c|}{ Speed } & \multicolumn{4}{c|}{ Size } \\
\multicolumn{1}{|c|}{ Unit } & MHz & Logic & \multicolumn{2}{|c|}{ IOBs } & \multicolumn{2}{c|}{ Slices } \\
\hline \hline Register File & 122.7 & $22 \%$ & 749 & $(87 \%)$ & 929 & $(4 \%)$ \\
Swap\&Rotate & 99.8 & $34 \%$ & 201 & $(23 \%)$ & 545 & $(2 \%)$ \\
Adress Generation & 141.3 & $50 \%$ & 188 & $(22 \%)$ & 274 & $(1 \%)$ \\
\hline
\end{tabular}

Table 6.2: Synthesis Results on Xilinx Virtex-IIp FPGA family

For the Register File the delay ratio caused by logic is only up to $22 \%$, for the Swap\&Rotate Unit this value reaches up to $35 \%$; interestingly, the logic needed for address generation makes $50 \%$ of the delay ratio.

Since these results are based on a straight-forward implementation and do not make use of any special feature offered by the Virtex-IIp FPGA family, it can be expected that an optimized design and proper floorplanning will push the speed even for the FPGA-based design into the speed range of slower ASIC designs (155 MHz).

When targetting usual $0.18 \mu \mathrm{m}$ ASIC technologies a speed-up of 100 to 200 percent compared to the FPGA-based solution can be expected; this is not only an educated guess among chip designers but also demonstrated by the numbers presented in Table 7.2 which show a speed-up range from 1.73 to 2.74 equalling 73 to 174 percent; this will give an estimated clock frequency of about 300 to $450 \mathrm{MHz}$ for the ASIC solution.

With fully custom chip design situation is even better considering that the main timing impact comes from signal routing following given signal as dictated by the used FPGA technology. Since also the logic circuitry can be tailor-made and does not need to be mapped to present logic cells, a speedup by a factor of 4 as compared to the FPGA technology is possible. Based on the used fabrication technology the estimated core logic speed for the CRYPTONITE architecture can reach $400 \mathrm{MHz}$ to $600 \mathrm{MHz}$.

The effective operating frequency will, however, be dictated by memory access time. Using aforementioned processes, $400 \mathrm{MHz}$ access rate is possible, but achieving higher frequencies is not likely. 


\subsection{Summary}

Within Section 6.1 of this chapter it was shown that the proposed CRYPTONITE architecture is able to perform the selected crypto algorithms at sufficient throughput.

The single algorithms were listed with complete cycle counts and additional explanations about the numbers where applicable; also, if an implementation makes assumptions about special units and configurations, this was remarked.

Within this paper, a clock rate of $400 \mathrm{MHz}$ was proposed. The reason for doing so was given in Chapter 5. In Section 6.2 it was proven that this rate indeed is possible when using custom chip fabrication; the presented numbers are promising enough that even with current ASIC technologies the postulated $400 \mathrm{MHz}$ can be reached.

This speed estimation was done by realizing selected parts of the architecture as VHDL modules, synthesizing and fitting them into the Xilinx Virtex-IIpro FPGA family and creating a timing analysis based on routing information and logic consumption as provided through the Synopsys development suite and the technology-specific programs provided by Xilinx.

These tests have proven the suitability of the CRYPTONITE architecture which will now be compared against existing hardware cores and programmable solutions in Chapter 7. 
6. IMPLEMENTATIONAL ASPECTS 


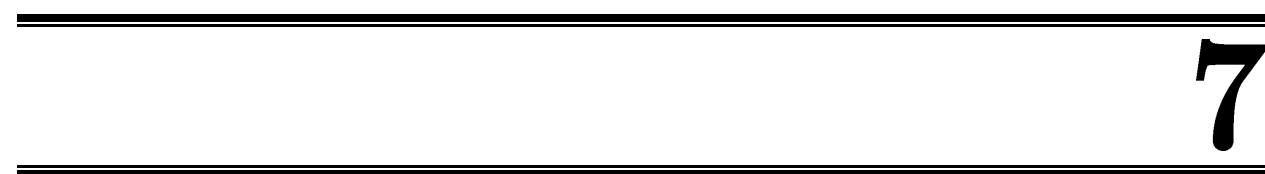

\section{Comparison of CRYPTONITE against Existing Solutions}

Comparing this architecture against existing solutions is not an easy task. First of all, almost no programmable solutions for solving cryptographic tasks exist. Most of the existing solutions are hard-coded, usually not even parametrizable which makes CRYPTONITE quite unique compared to its potential competitors.

Another obstacle are the numbers given by chip vendors. Hardly any company provides detailed performance data nor an insight into the architecture itself. Instead, an overall peak pipeline performance is given but no further information about the chip's architecture, especially its pipeline depth.

In some cases, these numbers only reflect the plain encryption performance and do not involve round key generation. Similarly, sometimes only numbers for the encryption process are given which makes comparison hard if not impossible: Chapters 2 and 3 have shown that round key generation is usually the by far more complex task compared to encryption itself as the IDEA example demonstrates; also, the fast AES implementation [45] indicates that decryption can be more complicated leading to slower performance. A comparison of a monolithic routine incorporating both directions and the round key generation against lightweighted solutions is quite difficult and easily draws a distorted picture.

Despite these problems, this chapter will try to compare the numbers for the CRYPTONITE architecture as presented in Chapter 6 against existing solutions and concepts. Based on this comparison a proper positioning of 
the proposed architecture within the field of crypto cores and programmable solutions will be worked out.

\subsection{Hardware Solutions \& IP Cores}

In the very beginning crypto algorithms were designed to easily fit into the respective time's technology. A good example for this is the DES algorithm which was developed to match 1970's technology. Also, in later times special hardware was designed to perform crypto operations fast and effectively. Although general purpose processors have evolved and today easily outperform yesterday's crypto hardware, dedicated hardware solutions are still far from being obsolete as dedicated hardware solutions naturally are faster than any general purpose processor solution.

For this comparison, a number of crypto cores was selected based on the data sheets provided by the respective companies. Selection reasons were speed, availability and technology numbers. As a side effect, the collected data also allows a comparison between cutting-edge hardware solutions like those offered by Amphion and SecuCore and established products like those included in the Broadcom and Hifn portfolios.

\subsubsection{Amphion Semiconductor Ltd.}

Amphion [9] is employed in a wide range of communication applications like speech encoding, video/imaging, channel coding and signal processing. One of their working fields also covers cryptography where Amphion has developed a set of AES encryption and decryption cores which are applicable to a wide range of fabrication technologies.

Fortunately, Amphion is one of the rare companies who publish straight numbers allowing not even performance but also technology comparison with CRYPTONITE. These numbers are presented in Table 7.2 which shows the hardware requirements of the various cores referring to selected target architectures. Table 7.1 compares the resulting throughput based on synthesis technology and core architecture. 
7.1. HARDWARE SOLUTIONS \& IP CORES

\begin{tabular}{|c|c|c|c|c|c|c|}
\hline \multirow[t]{2}{*}{ Device } & \multirow{2}{*}{$\begin{array}{l}\text { Key } \\
\text { Size }\end{array}$} & \multirow{2}{*}{$\begin{array}{c}\text { Cycles } \\
\text { per Op. }\end{array}$} & \multicolumn{4}{|c|}{ Data Rate in Mbits/s } \\
\hline & & & ASIC & APEX & Virtex-E & Virtex-Il \\
\hline \multicolumn{7}{|c|}{ "Encryption Devices } \\
\hline \multirow[t]{3}{*}{ CS5210 } & 128-bit & 44 & 581 & 226 & 275 & 341 \\
\hline & 192-bit & 52 & 492 & 191 & 233 & 289 \\
\hline & 256-bit & 60 & 426 & 166 & 202 & 250 \\
\hline CS5220 & 128-bit & 44 & 581 & 305 & 294 & 350 \\
\hline CS5230 & 128-bit & 11 & 2327 & 999 & 1061 & 1323 \\
\hline CS5240 & 128-bit & 1 & 25600 & $-\mathrm{n} / \mathrm{a}-$ & 9882 & 10880 \\
\hline \multicolumn{7}{|c|}{ Decryption Devices } \\
\hline \multirow[t]{3}{*}{ CS5250 } & 128-bit & 44 & 581 & 215 & 246 & 426 \\
\hline & 192-bit & 52 & 492 & 182 & 208 & 369 \\
\hline & 256-bit & 60 & 426 & 158 & 181 & 290 \\
\hline CS5260 & 128-bit & 44 & 581 & 233 & 264 & 290 \\
\hline CS5270 & 128-bit & 11 & 2327 & 727 & 896 & 1064 \\
\hline CS5280 & 128-bit & 1 & 25600 & $-\mathrm{n} / \mathrm{a}-$ & 8704 & 9344 \\
\hline
\end{tabular}

Table 7.1: CS52xx AES Core Performance

\subsubsection{CS5210/40 High Performance AES Encryption Cores}

The CS5210/40 family of AES encryption cores [10] are targeted towards a wide range of needs: Only the CS5210 is fully configurable and allows processing of any plaintext size together with any key size as defined within the AES specification, where the CS5220 is a shrunk down version of the CS5210 which supports only 128-bit key sizes but shares the same basic architecture. Plaintext data size is limited to 128 bits only.

CS5230 and CS5240 target high-performance areas and implement AES using pipelining techniques which boost the throughput by a factor of 4 (CS5230) and 44 (CS5240) achieving up to $25.6 \mathrm{GBit} / \mathrm{s}$ encryption rate compared to $581 \mathrm{MBit} / \mathrm{s}$ for the standard implementation as incorporated within CS5210 and CS5220.

These encryption cores have a basic interface which enables feeding in plaintext and key data on separate buses; similarly, the ciphertext output is available from an individual bus. The current chip status is presented through distinct status lines which signal if the chip is ready to accept new 


\begin{tabular}{|c|c|c|c|c|c|c|c|c|c|c|c|}
\hline \multirow[t]{2}{*}{ Device } & \multicolumn{2}{|c|}{ TSMC $0.18 \mu$} & \multicolumn{3}{|c|}{ Altera APEX-20K } & \multicolumn{3}{|c|}{ Xilinx Virtex-E } & \multicolumn{3}{|c|}{ Xilinx Virtex-II } \\
\hline & $\begin{array}{c}\text { Logic } \\
\text { Gates }\end{array}$ & $\begin{array}{l}\text { Clock } \\
\text { Speed }\end{array}$ & $\begin{array}{l}\text { Logic } \\
\text { Elem. }\end{array}$ & ESBs & $\begin{array}{l}\text { Clock } \\
\text { Speed }\end{array}$ & Slices & $\begin{array}{l}\text { Block } \\
\text { RAM }\end{array}$ & $\begin{array}{l}\text { Clock } \\
\text { Speed }\end{array}$ & Slices & $\begin{array}{l}\text { Block } \\
\text { RAM }\end{array}$ & $\begin{array}{l}\text { Clock } \\
\text { Speed }\end{array}$ \\
\hline CS5210 & $18.2 \mathrm{~K}$ & 200 & 1452 & 8 & 77.8 & 696 & 4 & 94.7 & 696 & $\overline{4}$ & 117.3 \\
\hline CS5220 & $14.8 \mathrm{~K}$ & 200 & 869 & 8 & 105 & 421 & 4 & 101 & 403 & 4 & 102.2 \\
\hline CS5230 & $27.0 \mathrm{~K}$ & 200 & 1167 & 20 & 85.9 & 573 & 10 & 91.2 & 573 & 10 & 113.7 \\
\hline CS5240 & $203.0 \mathrm{~K}$ & 200 & & $-\mathrm{n} / \mathrm{a}-$ & & 2397 & 100 & 77.2 & 2181 & 100 & 85 \\
\hline CS5250 & $19.2 \mathrm{~K}$ & 200 & 1560 & 8 & 74.1 & 745 & 4 & 84.7 & 746 & 4 & 100 \\
\hline CS5260 & $16.4 \mathrm{~K}$ & 200 & 1176 & 11 & 80.4 & 549 & 4 & 91 & 549 & 4 & 100 \\
\hline CS5270 & $34 \mathrm{~K}$ & 200 & 1481 & 20 & 62.5 & 778 & 10 & 77 & 778 & 10 & 91.5 \\
\hline CS5280 & $283 \mathrm{~K}$ & 200 & & $-\mathrm{n} / \mathrm{a}-$ & & 4626 & 100 & 68 & 3998 & 100 & 73 \\
\hline
\end{tabular}

Table 7.2: Technology and Performance Comparison for Amphion Cores 
plaintext or key data, if the encryption process is still running, or if the ciphertext data is valid.

\subsubsection{CS5250/80 High Performance AES Decryption Cores}

The CS5250/80 family of decryption cores [11] is the counterpart to the aforementioned encryption core family and incorporates the same basic design. However, compared to encryption the numbers show a decrease of throughput of up to $30 \%$ for decryption with FPGAs as target technologies together with an increase in size of up to $93 \%$.

If realized using ASIC technology the throughput remains the same but also an increase in chip size of up to $40 \%$ can be monitored.

\subsubsection{Broadcom Corporation}

Broadcom [25] is specialized in broadband communications and networking of voice, video, and data services. They offer a wide product range of integrated silicon solution targeting network access using various technologies (optical, wireless, xDSL) and also data security. For the security market Broadcom offers a variety of cryptographic and security processors targeted towards high-bandwidth networks and e-commerce. Broadcom's portfolio holds integrated devices including PCI-bus interface which can be attached to any PCI-based computer system but also single-chip solutions with an arbitrary interface allowing the chips to be connected to almost any processor or I/O subsystem using minimal glue logic.

Although Broadcom provides quite informative data sheets $[16,15,18$, $17,20,19,22,21,23,24]$ these mostly focus on the PCI aspect and do not reveal any architecture information of their crypto cores. Also, the given numbers mostly refer to combined operation like encryption plus digesting like 3DES+MD5 according to data packet sizes as transmitted over network. This information is collected in Table 7.3.

Table 7.4 presents less individual data and compares raw throughput showing the core's theoretical maximum speed against system throughput giving a vague idea about how much speed is lost for network protocol processing. A most interesting number, the sustained performance, is unfortunately given for only one chip, the BCM5802. Since the data sheets claim that BCM5801, BCM5802, BCM5805, and BCM5820 share basically the 
same architecture $[15,17,19,21]$, the sustained performance was calculated from the system performance for BCM5801, BCM5805, and BCM5820 based on the relation between system and sustained performance given for BCM5802.

Since only a summary throughput of combined operations like 3DES together with MD5 is given, it is not possible to calculate cycle numbers for individual algorithms which means that these processors cannot be directly compared to the CRYPTONITE architecture.

\subsubsection{Corrent Corporation}

The Corrent Corporation [36] is specialized in network security solutions for Gigabit-speed encryption in e-commerce (SSL) and virtual private networks (IPsec) applications [37] and gained big respect by earning the Microprocessor Report Analysts' Choice Award for Best Security Processor of 2001 $[89,90]$ for their CR7020 SSL Security Processor.

However, Corrent was unable to provide the necessary data sheets allowing a deeper insight into the architecture and its capabilities. Based on [89] the CR7020 compares to Broadcom's BCM5840 and Hifn's 8154 but achieves a peak IPsec bandwidth of $3.8 \mathrm{~GB} / \mathrm{s}$ and up to $5000 \mathrm{RSA}$ operations per second. It supports AES, DES, 3DES and ARC4 encryption together with SHA-1 and MD5 hashing functions and random number generation and can be interfaced to 33 and $66 \mathrm{MHz}$ PCI bus systems. For the lack of proper datasheets this chip - although very interesting - was omitted from further comparison.

\subsubsection{Hi/fn, Inc.}

Hifn [63] is a supplier of compression, encryption, authentication, and application recognition technologies especially targetting virtual private network and e-commerce markets. They offer a broad range of products of which the security-related processors were selected for comparison.

Much like Broadcom Hifn provides detailed data sheets [53, 54, 55, 56, $57,58,59,60,61,62]$ which concentrate on the PCI aspect but hardly give any insight into the architecture besides the information that the crypto engines use pipelining technologies. Also, the core performance is not given for all architectures but only the network throughput: As for 7814 and 7854 


\begin{tabular}{|ccc|rrrrrrrrr|}
\hline Device & PCI & Config & Core & \multicolumn{4}{|c|}{ Throughput/Mbps @ Packet Size/Bytes } \\
& Speed & & Speed & 64 & 128 & 256 & 512 & 1024 & 2048 & 5120 & 10240 \\
\hline \hline BCM5801 & $33 \mathrm{MHz}$ & - & 66 & 41 & $\mathrm{n} / \mathrm{a}$ & 97 & 127 & 149 & 162 & $\mathrm{n} / \mathrm{a}$ & $\mathrm{n} / \mathrm{a}$ \\
& $66 \mathrm{MHz}$ & - & 66 & 43 & $\mathrm{n} / \mathrm{a}$ & 112 & 152 & 186 & 205 & $\mathrm{n} / \mathrm{a}$ & $\mathrm{n} / \mathrm{a}$ \\
BCM5802 & $33 \mathrm{MHz}$ & - & 33 & 28 & $\mathrm{n} / \mathrm{a}$ & 67 & 89 & 104 & 113 & $\mathrm{n} / \mathrm{a}$ & $\mathrm{n} / \mathrm{a}$ \\
BCM5805 & & encrypt & 50 & 44 & 75 & 110 & 145 & 168 & 198 & 198 & 204 \\
& & decrypt & 50 & 75 & 101 & 133 & 162 & 179 & 194 & 201 & 205 \\
BCM5820 & $33 \mathrm{MHz}$ & inbound & 33 & 26 & 45 & 62 & 84 & 101 & 111 & $\mathrm{n} / \mathrm{a}$ & $\mathrm{n} / \mathrm{a}$ \\
& & outbound & 33 & 45 & 64 & 81 & 98 & 109 & 116 & $\mathrm{n} / \mathrm{a}$ & $\mathrm{n} / \mathrm{a}$ \\
& & inbound & 90 & 55 & 95 & 132 & 178 & 211 & 233 & $\mathrm{n} / \mathrm{a}$ & $\mathrm{n} / \mathrm{a}$ \\
& & outbound & 90 & 77 & 117 & 154 & 191 & 219 & 236 & $\mathrm{n} / \mathrm{a}$ & $\mathrm{n} / \mathrm{a}$ \\
BCM5820 & $66 \mathrm{MHz}$ & inbound & 66 & 50 & 88 & 124 & 168 & 201 & 233 & $\mathrm{n} / \mathrm{a}$ & $\mathrm{n} / \mathrm{a}$ \\
& & outbound & 66 & 87 & 119 & 158 & 193 & 218 & 233 & $\mathrm{n} / \mathrm{a}$ & $\mathrm{n} / \mathrm{a}$ \\
& & inbound & 90 & 55 & 97 & 138 & 193 & 240 & 269 & $\mathrm{n} / \mathrm{a}$ & $\mathrm{n} / \mathrm{a}$ \\
& & outbound & 90 & 87 & 119 & 163 & 212 & 248 & 274 & $\mathrm{n} / \mathrm{a}$ & $\mathrm{n} / \mathrm{a}$ \\
\hline
\end{tabular}

Table 7.3: Broadcom BCM58xx performance regarding packet size 


\begin{tabular}{|c|c|c|c|c|c|c|c|}
\hline \multirow[b]{2}{*}{ Device } & \multicolumn{2}{|c|}{ Supported Algorithms } & \multirow{2}{*}{$\begin{array}{l}\text { Speed } \\
(\mathrm{MHz})\end{array}$} & \multicolumn{4}{|c|}{ Performance (MBit/s) } \\
\hline & Crypto & Hash & & ctrraw & system & sus & ained \\
\hline$\overline{\text { BCM5801 }}$ & 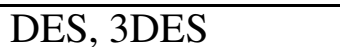 & SHA-1, MD5 & 33 & 320 & $\overline{200}$ & & 100 \\
\hline BCM5802 & DES, 3DES & SHA-1, MD5 & 33 & 150 & 100 & & 50 \\
\hline BCM5805 & DES, 3DES & SHA-1, MD5 & 33 & 240 & 200 & & 100 \\
\hline BCM5820 & DES, 3DES, ARC4 & SHA-1, MD5 & 33 & 330 & 290 & & 145 \\
\hline BCM5821 & DES, 3DES, ARC4 & SHA-1, MD5 & 125 & $\mathrm{n} / \mathrm{a}$ & $\begin{array}{l}470 \\
600\end{array}$ & $\begin{array}{c}\text { (3DES/SHA1) } \\
\text { (ARC4) }\end{array}$ & $\begin{array}{l}\mathrm{n} / \mathrm{a} \\
\mathrm{n} / \mathrm{a}\end{array}$ \\
\hline BCM5840 & DES, 3DES & SHA-1, MD5 & $\mathrm{n} / \mathrm{a}$ & $\mathrm{n} / \mathrm{a}$ & 2400 & (3DES/SHA1) & $\mathrm{n} / \mathrm{a}$ \\
\hline
\end{tabular}

Table 7.4: Broadcom BCM58xx performance data 
only the vague information that "AES [has] somewhat greater performance than $3 D E S$ " [56] is given, for $7851,8 \times 65$ and 8154 no core performance numbers at all are provided.

For this reason, the core performance data as provided through Hifn's data sheets is listed in Table 7.5. From these given values rough cycle count numbers as shown in Table 7.6 were calculated to allow further comparison using the formula

$$
\text { cycles }=\frac{(\text { CoreSpeed } / \text { MHz } z) * \text { DataChunkSize } / \text { Bits })}{\text { Performance } / M B i t / s}
$$

Although these numbers might not reflect the exact cycle count they are a good base for architecture comparison. It must be noted, however, that the numbers for AES (where applicable) assume 128-bit data and key size (AES 128/128) and are based on the 3DES throughput numbers since the data sheets claim the AES performance to be "somewhat greater than 3DES" [56]. These numbers have to be taken with caution.

No numbers were calculated for RC4 since neither key nor data size were given; similarly the $80 \mathrm{xx} / 81 \mathrm{xx}$ family of Secure Session Processors was omitted from this list as no detailed data was provided.

\subsubsection{NetOctave, Inc.}

NetOctave [84] is specialized in building security processors and security accelarator boards targeting Secure Sockets Layer (SSL), IP Security (IPSec) and IP Storage markets. With their NSP2000 [79, 80] and NSP3200 $[82,81]$ NetOctave provides dedicated PCI-enabled crypto processors tailored towards direct network attachment to support SSL (NSP2000 [80]) and IPsec (NSP3000 [81]) secured connections. The NSP series of processors was presented to a wider audience on the Communications Design Conference (CDC) 2001 through [99] and [100].

\subsubsection{Performance Data}

Also NetOctave does not provide sufficient data for an architectural comparison, only maximum numbers for network throughput are given. which reaches up to $4.8 \mathrm{GBit} / \mathrm{s}$ for the NSP3200 Security Processor [82] with 650 MBit/s for combined 3DES/SHA-1 or 3DES/MD5 running on a NSP300x IPsec Security Accelerator Board [81]. For the NSP200x SSL Security Accelerator Board [80] only the core performance of $650 \mathrm{MBit} / \mathrm{s}$ (NSP2000/2) 


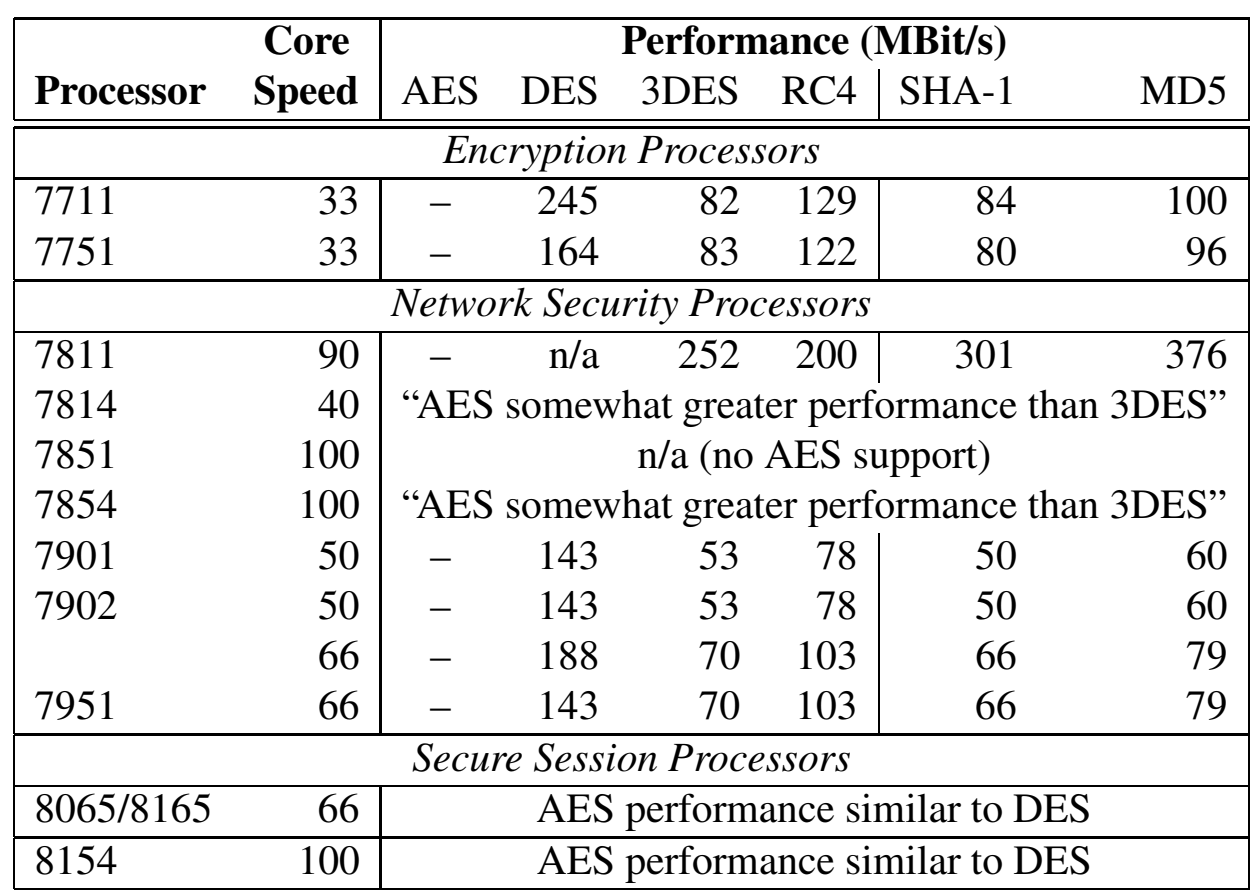

Table 7.5: Performance Data of Hifn Processors

\begin{tabular}{|l|rrrr|}
\hline & \multicolumn{4}{|c|}{ Cycle Count for } \\
Algorithm & $\mathbf{7 7 1 1}$ & $\mathbf{7 7 5 1}$ & $\mathbf{7 8 x x}$ & $\mathbf{7 9 x x}$ \\
\hline \hline AES & n/a & n/a & 46 & n/a \\
DES & 9 & 13 & n/a & 22 \\
3DES & 26 & 25 & 23 & 61 \\
SHA-1 & 201 & 211 & 153 & 512 \\
MD5 & 169 & 176 & 123 & 427 \\
\hline
\end{tabular}

Table 7.6: Algorithm cycle counts computed from given performance data 
7.1. HARDWARE SOLUTIONS \& IP CORES

\begin{tabular}{|c|c|c|c|c|}
\hline \multirow[b]{2}{*}{ Product } & \multicolumn{2}{|c|}{ Supported Algorithms } & \multicolumn{2}{|c|}{ Performance } \\
\hline & Crypto & Hash & Core & Network \\
\hline \multicolumn{5}{|c|}{ Data provided by NetOctave } \\
\hline NSP2000B & 3DES, ARC4 & MD5, SHA1 & $650 \mathrm{MBit} / \mathrm{s}$ & $\mathrm{n} / \mathrm{a}$ \\
\hline NSP2002B & 3DES, ARC4 & MD5, SHA1 & 650MBit/s & $\mathrm{n} / \mathrm{a}$ \\
\hline NSP2004B & 3DES, ARC4 & MD5, SHA1 & 1300MBit/s & $\mathrm{n} / \mathrm{a}$ \\
\hline NSP3000B & 3DES & MD5, SHA1 & $650 \mathrm{MBit} / \mathrm{s}$ & $\mathrm{n} / \mathrm{a}$ \\
\hline & AES, 3DES & MD5, SHA1 & $\mathrm{n} / \mathrm{a}$ & 4.8GBit/s \\
\hline \multicolumn{5}{|c|}{ Data provided by Chipcenter } \\
\hline $\begin{array}{l}\text { NSP2000 } \\
@ 100 \mathrm{MHz}\end{array}$ & 3DES & $\begin{array}{l}\text { MD5 } \\
\text { SHA }\end{array}$ & $\begin{array}{l}1000 \mathrm{MBit} / \mathrm{s} \\
1190 \mathrm{MBit} / \mathrm{s} \\
2000 \mathrm{MBit} / \mathrm{s}\end{array}$ & $\begin{array}{l}\mathrm{n} / \mathrm{a} \\
\mathrm{n} / \mathrm{a} \\
\mathrm{n} / \mathrm{a}\end{array}$ \\
\hline
\end{tabular}

Table 7.7: Performance Data for NetOctave SSL and IPsec Processors

and 1.3 GBit/s (NSP2004) are given. Chipcenter [79] gives more detailed but different numbers which have also been added into Table 7.7.

Although a white paper about NetOctave's FlowThrough Security Architecture [83] is provided, it does not give a detailed description of the architecture but contains only basic information about the various modules integrated into one chip.

Similar to Broadcom, NetOctave only provides numbers for combined operation which do not allow to calculate approximate cycle counts for individual operations as needed for comparison with the CRYPTONITE architecture.

\subsubsection{OpenCores.org}

OpenCores [86] follows the same ideology as the Open Software Foundation (OSF) [85]. Where OSF provides free software, OpenCores focuses on free hardware cores. Through their pages, OpenCores offers a wide range of Verilog and VHDL hardware models in different development stages; from the crypto cores section only the DES core [128] is readily developed and tested on various hardware achitectures. In addition, the IDEA [3] core is developed far enough to present at least minimal numbers. Currently under development are AES/Rijndael [136], RC4 [134], Twofish [5] and 


\begin{tabular}{|llrl|}
\hline \multicolumn{1}{|c}{$\begin{array}{c}\text { Hardware } \\
\text { Platform }\end{array}$} & \multicolumn{1}{c|}{ Size } & Speed (MHz) \\
Core & Processing \\
\hline \hline \multicolumn{4}{|c|}{ Area Optimized } \\
\hline 0.18 $\mu$ m UMC ASIC & 3k Gates & 155 & 9.68 \\
Altera APEX 20K & 1106 Icells & 27 & 1.68 \\
Altera FLEX 10K50 & 1283 Icells & 43 & 2.68 \\
\hline \multicolumn{4}{|c|}{ Performance Optimized } \\
\hline 0.18 $\mu$ m UMC ASIC & 28k Gates & 290 & 18.13 \\
Altera APEX 20K & 6688 Icells & 53 & 3.31 \\
Altera FLEX 10K50 & 6485 Icells & 76 & 4.75 \\
\hline
\end{tabular}

Table 7.8: OpenCores DES Performance Data

SHA-256 [135] cores as well as a monolithic crypto accelerator supporting AES/Rijndael, Twofish, and SHA-1 [120]. Since these cores are mostly still in planning stage they are listed for completeness but will not be further investigated.

\subsubsection{OpenCores DES Core}

The DES core has already been implemented and tested on multiple hardware platforms. It takes 16 cycles to perform a full encryption or decryption which implies that each round takes one cycle, input/output permutation are embedded into the data path. The performance data is shown in Table 7.8. For this core the bits-per-cycle ratio equals 4.

\subsubsection{OpenCores IDEA Core}

Although announced for quite some time, this core still is not finished. However, it has already been implemented in hardware and is said to achieve a throughput of $177 \mathrm{MBit} / \mathrm{s}$, further information about the core speed and the needed cycles per IDEA encryption or decryption is omitted. The given diagrams are no help since they are somewhat contradictory: The core's block diagram seems to receive key and data 16-bit-wise, similarly the cipher text is written out in items of 16-bit. The IDEA Machine, however, as main 
7.1. HARDWARE SOLUTIONS \& IP CORES

\begin{tabular}{|l|lrrr|}
\hline Core & $\begin{array}{l}\text { Throughput } \\
\text { (GBit/s) }\end{array}$ & Cycles & $\begin{array}{c}\text { Data Chunk } \\
\text { Size }\end{array}$ & $\begin{array}{c}\text { Bits per } \\
\text { Cycle }\end{array}$ \\
\hline \hline AES-128 & 1.94 & 11 & 128 & 11.6 \\
DES & 2. & $>5$ & 64 & 12.04 \\
3DES & 0.67 & $<16$ & 64 & 4.01 \\
MD5 & 1.25 & 68 & 512 & 7.53 \\
SHA-1 & 1.01 & 84 & 512 & 6.10 \\
SHA-256 & 1.25 & 68 & 512 & 7.53 \\
\hline
\end{tabular}

Table 7.9: SecuCore Performance Data

functional unit seems to perform the complete IDEA operation within one cycle.

Since no additional documentation is given, this core is omitted from further comparison due to incomplete and contradictory data.

\subsubsection{Secucore Consulting Services}

SecuCore [116] is a provider of high performance hardware core models targeting data security. They offer a broad variety of cores for different crypto algorithms, namely AES/Rijndael [112], DES and 3DES [113], MD5 [114], and SHA-256 [115] which deliver high performance using a $0.18 \mu \mathrm{m}$ ASIC technology.

\subsubsection{Performance Overview}

According to the data sheets, SecuCore used TMSC's $0.18 \mu \mathrm{m}$ ASIC technology running at $166 \mathrm{MHz}$ to benchmark their cores. Also, most of the data sheets provide the number of cycles needed to perform the crypto operation on the algorithm's specific data chunk size. For DES and 3DES, however, these values were not provided and had to be calculated from throughput, core speed and data size.

\subsubsection{Zyfer, Inc.}

Zyfer's [140] main area of operation is precision synchronization devices as used for communication, networks and military applications with focus on 
secure and robust data transfer through ground and satellite links. One of their products is the SKP-100 Gigabit Network Security Processor [141]

\subsubsection{SKP-100 Gigabit Network Security Processor}

The SKP-100 is currently under development, so Zyfer publishes only a short product information presenting minimal facts. It supports AES/Rijndael-128/128, 3DES in 2- and 3-key configuration and plain DES, all in EBC and CBC mode. For authentication, MD5, SHA-1, and SHA-256 hash algorithms are supported.

For 3DES and Rijndael the product information claims that full duplex OC-48 speed (2.4 GBit/s) is achieved, but since this number is also given as "pipe speed" it can be safely assumed that that these algorithms are not implemented single-cycle.

Since further insight into the architecture like pipe length or number and structure of processing units are given, no comparative values can be calculated.

\subsection{Programmable Solutions}

Fully programmable crypto processors are still a novel concept: Until now almost nobody has entered this field and so far only one fully programmable and algorithm independent machine, the CryptoManiac [131], is known. Besides, a microprogrammable processor targeted towards IDEA exists, the PLD001 Cryptoprocessor [67]. Although it was developed with respect to IDEA it is not clearly IDEA specific and can be considered as another programmable solution.

\subsubsection{CryptoManiac}

CryptoManiac appeared in mid 2001 and is a 4-wide 32-bit VLIW architecture. It basically consists of 4 functional units operating on a commonly used data memory which are part of a 4-stage pipeline. To keep this pipeline filled a 16-entry branch target buffer is used for branch prediction. Figure 7.1 gives an overview over the CryptoManiac architecture. 
CryptoManiac explicitly supports communication with a host processor through a queue mechanism providing input and output data queues. A request scheduler is responsible for distributing incoming requests in order of appearance. It also offers a special keystore interface to hold key data and substitution tables.

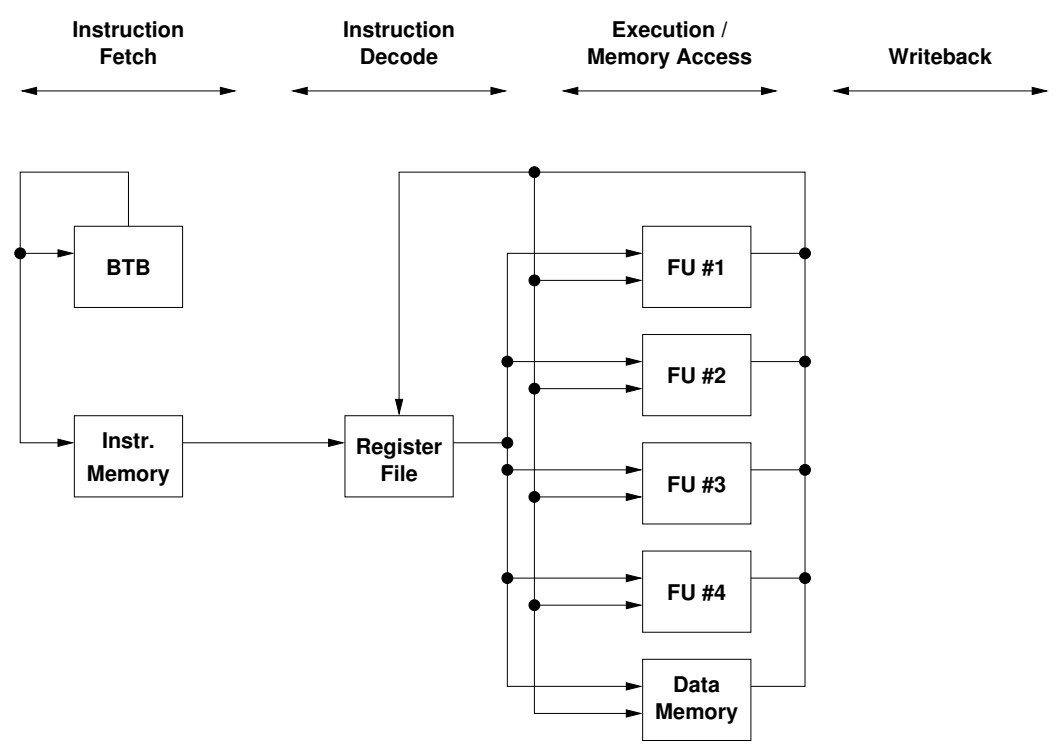

Figure 7.1: CryptoManiac Architecture Overview

The heart of the CryptoManiac architecture is the four functional units as depicted in Figure 7.2 which are less complex than CRYPTONITE's arithmetic unit, but are able to perform up to three sequential operations within one cycle.

\subsubsection{Conceptual differences to CRYPTONITE}

Although there are some similarities between CRYPTONITE and CryptoManiac concerning the concept of multiple functional units or VLIW, the architectures are quite different at a closer look.

Firstly, CRYPTONITE is a single-cycle execution architecture. It does not allow multi-cycle operations like CryptoManiac with its instructioncombining model. The reason to do so was mainly for speed reasons but 


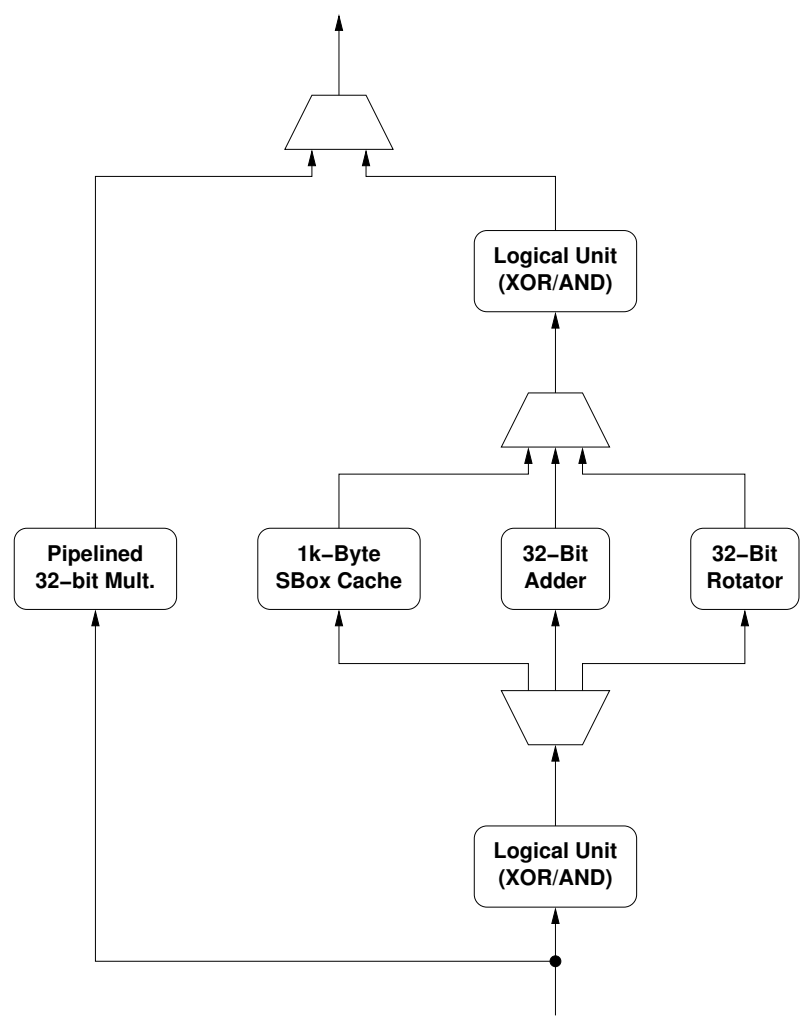

Figure 7.2: CryptoManiac's functional unit

to a smaller degree also to ease compiler design, namely resource allocation. The drawback of single-cycle execution is that it puts great pressure on the hardware design to ensure that all functions can be performed within one cycle.

On the level of functional units data access and data processing are strictly separated ${ }^{1}$ in CRYPTONITE. All data like input data, keys, tables, or S-Boxes is held in memory, as opposite to CryptoManiac's functional units which employ S-Box caches. On the other hand, CRYPTONITE's arithmetic units are more powerful and allow quite complex monolithic operations to support the needs of advanced crypto algorithms.

${ }^{1}$ with the exception of the DES unit which is placed in the memory unit as explained in Section 5.8.4 
7.2. Programmable Solutions

\begin{tabular}{|l|c|c|rrrr|}
\hline & Alpha & CRYPTO- & \multicolumn{4}{|c|}{ CryptoManiac } \\
& $\mathbf{2 1 1 6 4}$ & NITE & 4WC & 3WC & 2WC & 4WNC \\
\hline \hline Blowfish & 9.58 & n/a & 4 & 4 & 6 & 5 \\
3DES & 23.56 & $2 / 3$ & 7 & 8 & 9 & 12 \\
IDEA & 91.95 & 11 & 14 & 14 & 17 & 15 \\
Mars & 28.86 & $\mathrm{n} / \mathrm{a}$ & 9 & 9 & 9 & 10 \\
RC4 & 11.49 & $\mathrm{n} / \mathrm{a}$ & 8 & 8 & 8 & 9 \\
RC6 & 23.24 & $7+3$ & 7 & 7 & 7 & 9 \\
AES/Rijndael & 33.84 & $8 / 7$ & 9 & 11 & 17 & 10 \\
Twofish & 27.36 & n/a & 7 & 8 & 11 & 8 \\
\hline
\end{tabular}

Table 7.10: Kernel loop cycle counts per round

Another difference shows up together with memory access: Where CryptoManiac allows all four functional units to access a common data memory, CRYPTONITE has separate memory units for both ALUs. Communication with external units is handled through request pipelines within CryptoManiac; as of now, the CRYPTONITE architecture only provides a raw interrupt-based communication interface allowing external units to put CRYPTONITE on hold while accessing the internal memory to update or read out values. This means that an external control processor handling the network data stream has to frequently update CRYPTONITE's data memory to provide new and read out processed data.

\subsubsection{Performance Data}

For CryptoManiac quite a number of evaluations were done covering computation speed, power dissipation, timing results, and on-chip area requirements. The speed evaluation was compared against a modern general purpose processor, the Alpha 21264; also, the numbers for the CRYPTONITE architecture were added where applicable. The results of these comparisons are shown in Table 7.10 for performance data and Table 7.11 for physical parameters.

Unfortunately, the cycle count is only given for an algorithm's round and not for the entire encryption process of a data block which makes throughput calculation a bit problematic. Based on Table 6.1 the ratio between round 


\begin{tabular}{|l|llll|}
\hline & \multicolumn{4}{|c|}{ CryptoManiac } \\
& 4W Comp. & 3W Comb. & 2W Comb. & 4W N-Comb. \\
\hline \hline Chip Speed & $2.78 \mathrm{~ns}$ & $2.66 \mathrm{~ns}$ & $2.54 \mathrm{~ns}$ & $2.75 \mathrm{~ns}$ \\
& $360 \mathrm{MHz}$ & $376 \mathrm{MHz}$ & $394 \mathrm{MHz}$ & $364 \mathrm{MHz}$ \\
Chip Size & $1.93 \mathrm{~mm}^{2}$ & $1.77 \mathrm{~mm}^{2}$ & $1.59 \mathrm{~mm}^{2}$ & $1.69 \mathrm{~mm}^{2}$ \\
Power & $606.37 \mathrm{~mW}$ & $593.51 \mathrm{~mW}$ & $568.50 \mathrm{~mW}$ & $586.86 \mathrm{~mW}$ \\
Consumption & & & & \\
\hline
\end{tabular}

Table 7.11: Timing and Area Results for CryptoManiac

cycle count and total cycle count was calculated; these ratios serve as a multiplication factor for the CryptoManiac kernel loop counts as provided through Table 7.10. Table 7.12 shows the comparative numbers based on this calculation.

These numbers have to be taken with extreme caution and are most likely too optimistic since [131] does not mention round key generation at all. As for CRYPTONITE code AES round key generation is responsible for about $30 \%$ of the cycle count; for a (3)DES implementation using singleoperation instructions (as opposed to the monolithic instructions employed within CRYPTONITE) round key generation causes about $16 \%$ of the overall cycle count. To take this into account, corrected values were calculated for 3DES and AES by multiplying the ratio factor by 1.16 and 1.30 respectively.

It must be further noticed that only the CryptoManiac's 4WC configuration running at $360 \mathrm{MHz}$ was considered.

\begin{tabular}{|c|c|c|c|c|c|c|}
\hline \multirow[b]{2}{*}{ Algorithm } & \multirow{2}{*}{\multicolumn{2}{|c|}{$\begin{array}{l}\text { Round } \\
\text { Cycles }\end{array}$}} & \multirow[b]{2}{*}{ Ratio } & \multirow{2}{*}{$\begin{array}{c}\text { Total } \\
\text { Cycles }\end{array}$} & \multicolumn{2}{|c|}{ Throughput } \\
\hline & & & & & Bits/Cycle & MBit/s \\
\hline 3DES & 7 & * & 48 & 336 & 0.19 & 68 \\
\hline 3DES corr. & 7 & * & 56 & 392 & 0.16 & 59 \\
\hline IDEA & 14 & * & $8.18=$ & 115 & 1.11 & 400 \\
\hline RC6 & 7 & $*$ & $20.6=$ & 144 & 0.89 & 320 \\
\hline AES-128/128 & 9 & & $10=$ & 90 & 1.42 & 511 \\
\hline AES-128/128 corr. & 9 & * & 13 & 130 & 0.98 & 353 \\
\hline
\end{tabular}

Table 7.12: Estimated Throughput for CryptoManiac running at $360 \mathrm{MHz}$ 


\subsubsection{PLD001 Cryptoprocessor}

A crypto processor designed towards IDEA and RSA was developed by Jüri Pöldre during 1994 to 1997 [67]. Like this work it followed a very similar approach by analyzing distinct algorithms and tayloring an architecture towards these algorithms. The PLD001 is a microprogrammable architecture allowing different ALU configurations as needed for certain processing steps within IDEA computation.

The analysis of RSA and IDEA led to a quad-partite ALU as shown in Figure 7.3 consisting of four 24-bit wide units. For IDEA these units are configured as two 16-bit multipliers. Since each 24-bit block can perform an $8 * 16$-bit multiplication, two such multipliers do the needed $16 * 16$ bit multiplication in one cycle. If long modular calculation is needed, the ALU can be configured as an $8 * 96$-bit multiplier or 96-bit adder/negator with additional carry logic as shown in Figure 7.4. This configuration is called calculation mode. The complete data path within PLD001's ALU is depicted in Figure 7.5.
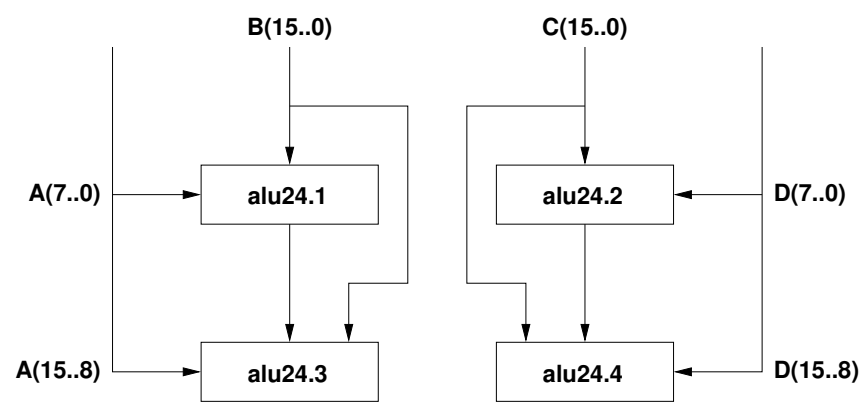

Figure 7.3: IDEA configuration

Besides ALU configuration, PLD001 microprograms allow access to the internal memory and selection of desired logical and arithmetic operations. Memory access is controlled by the so-called ALU control structure as shown in Figure 7.6. The INDEX_CALC unit is capable of either addressing 6-bit entities inside the 768-bit data registers or loading parts of the 768-bit memory into the 24-bit register ER. Logic operations between between immediate values, index register, and ER may apply. Arith- 


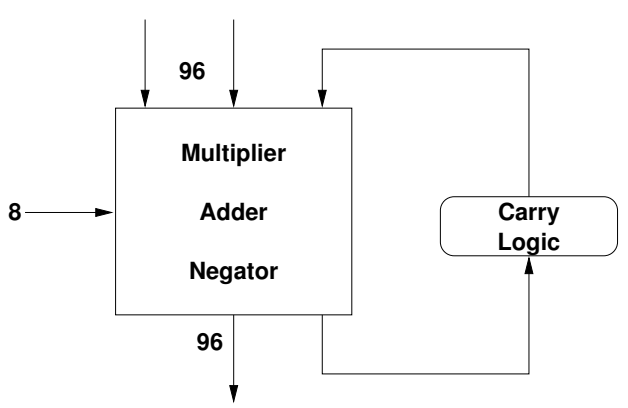

Figure 7.4: Calculation Mode

metic commands only operate on long-data registers and are executed in the ALU_SEQUENCER unit in parallel with index calculation.

PLD001 also supports so-called high-level commands which are basically calls to microcode programs allowing reusable complex operations. 32 of these are fall under the category external commands which means that these can be directly selected by applying the proper bit pattern to dedicated input pins.

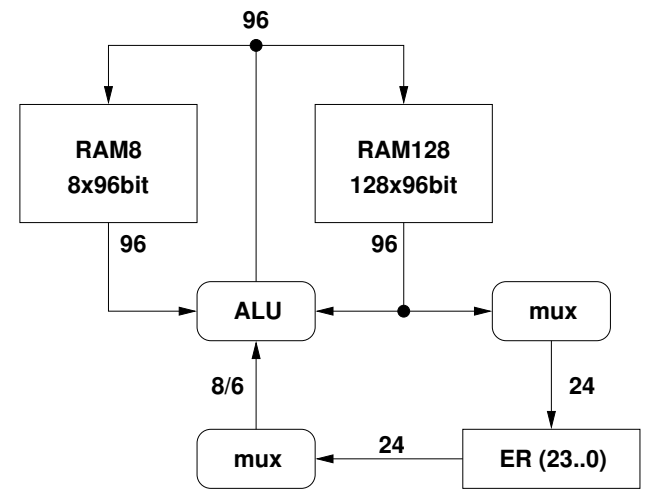

Figure 7.5: Data flow within the PLD001 ALU

\subsubsection{Performance}

[67] gives mainly numbers for IDEA calculation. According to this document it takes 50 clock cycles per IDEA transformation equalling $32 \mathrm{MBit} / \mathrm{s}$ 


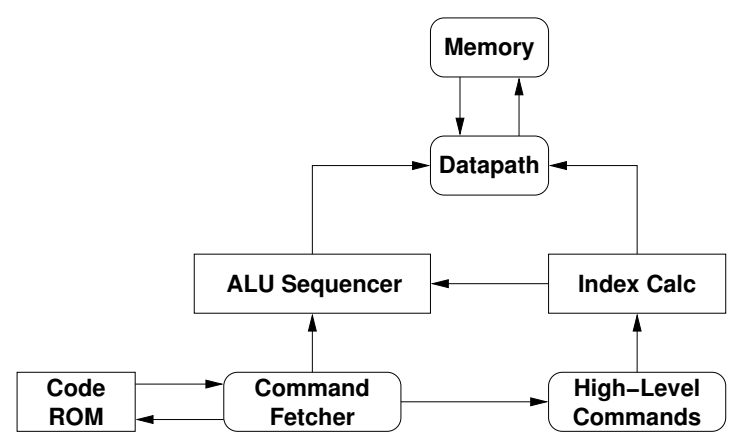

Figure 7.6: PLD001 ALU control structure

at the denoted $25 \mathrm{MHz}$ operating speed. RSA key exchange is said to take about $0.5 \mathrm{~s}$ which equals 2 key exchanges per second. The values are summed up in Table 7.13.

\begin{tabular}{|ll|}
\hline Supported Algorithms & IDEA and RSA Key Exchange \\
Core Speed & $25 \mathrm{MHz}$ (tested) \\
& $20 \mathrm{MHz}$ (calculated) \\
Technology & ES2 $1.0 \mu \mathrm{m}$ and Xilinx FPGAs \\
Throughput & $32 \mathrm{MBit} / \mathrm{s}$ for IDEA \\
& $2 \mathrm{key}$ exchanges per second for RSA \\
\hline
\end{tabular}

Table 7.13: PLD001 Performance Data

\subsection{Algorithm Performance Comparison}

Within the last two sections many dedicated hardware solutions and two programmable solutions were presented. A comparation, however, is not an easy task since the various sources present quite different data. As certain parameters had to be estimated or calculated based on estimations, some values have to be taken with care but nevertheless allow a performance comparison between the CRYPTONITE architecture and its competitors. 


\subsubsection{AES-128/128 Performance}

Table 7.14 shows performance data for those crypto solutions supporting AES-128/128. The Amphion CS5240 was omitted from this list since it is a very special single-cycle solution achieving a throughput of $25600 \mathrm{MBit} / \mathrm{s}$ for the sake of an over 10-times bigger gate count. Figure 7.7 compiles this data into a more pictoral form.

\begin{tabular}{|l|rcc|}
\hline \multicolumn{1}{|c|}{ Architecture } & $\begin{array}{c}\text { Cycle } \\
\text { Count }\end{array}$ & $\begin{array}{c}\text { Speed } \\
(\mathrm{MHz})\end{array}$ & $\begin{array}{c}\text { Throughput } \\
\text { (MBit/s) }\end{array}$ \\
\hline \hline Amphion CS5220 & 44 & 200 & 581 \\
Amphion CS5230 & 11 & 200 & 2327 \\
Hifn 7854 & 46 & 100 & 280 \\
SecuCore AES-128 & 11 & 166 & 1931 \\
CryptoManiac (raw) & 90 & 360 & 511 \\
CryptoManiac (corr.) & 130 & 360 & 353 \\
\hline CRYPTONITE $(\leftrightarrow)$ & 80 & 400 & 640 \\
CRYPTONITE $(\rightarrow)$ & 70 & 400 & 731 \\
\hline
\end{tabular}

Table 7.14: AES-128/128 Performance Comparison

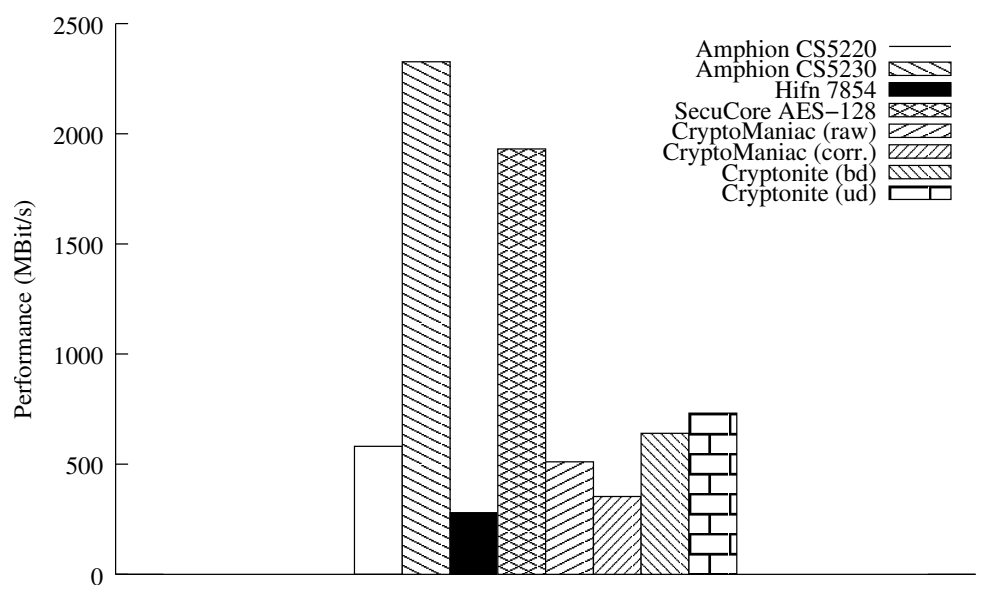

Figure 7.7: AES-128/128 Performance Comparison 
Besides CryptoManiac, the 44- and 11-cycle versions from Amphion (CS5220, CS5230), the 46-cycle version from Hifn (7854) and another 11cycle version from SecuCore (AES-128) which runs at slightly slower speed than the Amphion CS5230 are included which are positioned against the bidirectional $\leftrightarrow$ ) and unidirectional $(\rightarrow$ ) versions of CRYPTONITE's fast AES implementation [45].

It can be observed, that CRYPTONITE running at $400 \mathrm{MHz}$ outperforms the 44/46 cycle hardware implementations by a factor of 1.25 to 2.6; as expected, the 11-cycle versions outperform CRYPTONITE by a factor greater than 2.64, but it must been taken into account that these cores are extremely tailored towards the AES/Rijndael algorithm, which is especially true for the single-cycle solution CS5240. Compared against CryptoManiac CRYPTONITE shows an up to two times better performance.

\subsubsection{DES Performance}

As a result of the dedicated DES unit CRYPTONITE shows excellent crypto performance as listed in Table 7.15. It outperforms most of the cores by a factor of 1.18 to 5.12; only SecuCore DES shows again overwhelming performance and beats CRYPTONITE by a factor of 2.73. This data is graphically presented in Figure 7.8.

\begin{tabular}{|l|rcc|}
\hline Architecture & $\begin{array}{c}\text { Cycle } \\
\text { Count }\end{array}$ & $\begin{array}{c}\text { Speed } \\
(\mathrm{MHz})\end{array}$ & $\begin{array}{c}\text { Throughput } \\
\text { (MBit/s) }\end{array}$ \\
\hline \hline Hifn 7711 & 9 & 33 & 245 \\
Hifn 7751 & 13 & 33 & 164 \\
Hifn 790x & 22 & 50 & 143 \\
OpenCores DES & 16 & 155 & 620 \\
SecuCore DES & 5 & 166 & 1999 \\
\hline CRYPTONITE & 35 & 400 & 732 \\
\hline
\end{tabular}

Table 7.15: DES Performance Comparison 


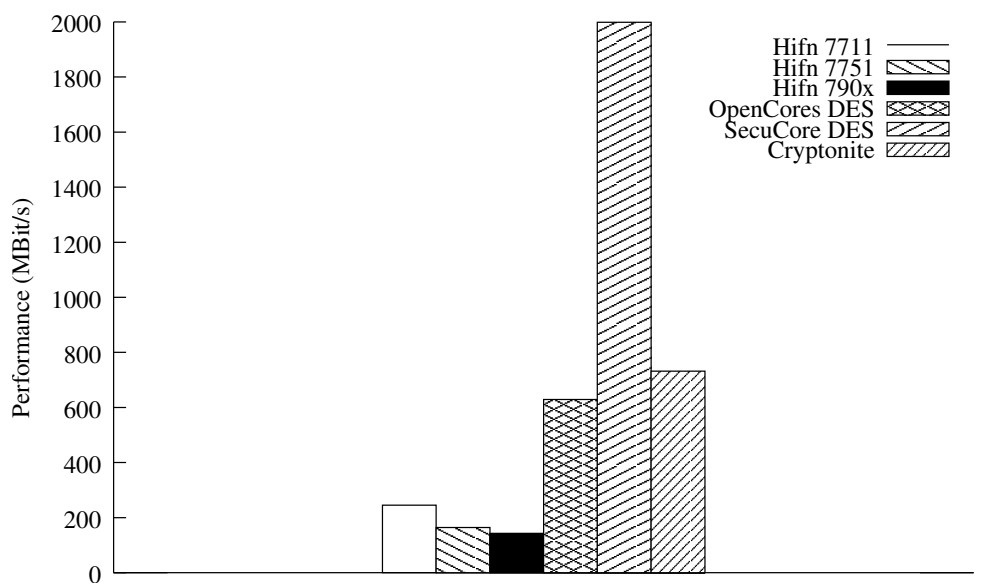

Figure 7.8: DES Performance Comparison

\subsubsection{DES Performance}

CRYPTONITE's great DES performance also shows up for 3DES where it outruns its hardware competitors by a factor up to 3.13 ; only the Hifn 7811 shows slighty better performance and reaches $103 \%$ of CRYPTONITE's throughput as listed in Table 7.16 and depicted in Figure 7.9. The comparison against the programmable competitor CryptoManiac shows the great benefit of CRYPTONITE's DES unit. Thanks to this unit CRYPTONITE outperforms CryptoManiac by factors up to 4.14 .

\begin{tabular}{|l|ccc|}
\hline \multicolumn{1}{|c|}{ Architecture } & $\begin{array}{c}\text { Cycle } \\
\text { Count }\end{array}$ & $\begin{array}{c}\text { Speed } \\
\text { (MHz) }\end{array}$ & $\begin{array}{c}\text { Throughput } \\
\text { (MBit/s) }\end{array}$ \\
\hline \hline Hifn 7711 & 26 & 33 & 82 \\
Hifn 7751 & 25 & 33 & 83 \\
Hifn 7811 & 25 & 90 & 252 \\
Hifn 790x & 61 & 50 & 78 \\
CryptoManiac (raw) & 336 & 360 & 68 \\
CryptoManiac (corr.) & 392 & 360 & 59 \\
\hline CRYPTONITE & 105 & 400 & 244 \\
\hline
\end{tabular}

Table 7.16: 3DES Performance Comparison 


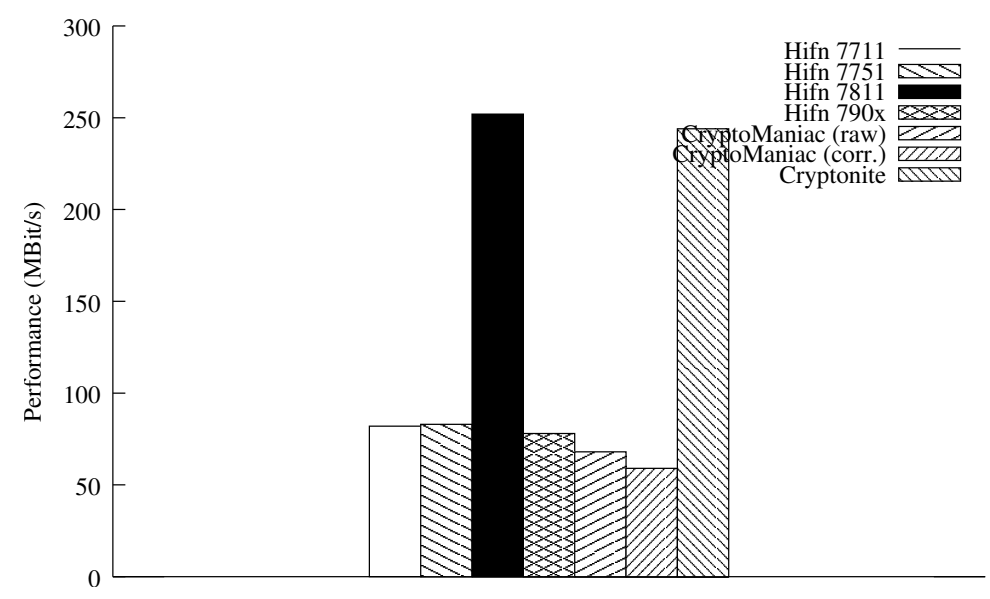

Figure 7.9: 3DES Performance Comparison

\subsubsection{IDEA Performance}

\begin{tabular}{|l|rrc|}
\hline Architecture & $\begin{array}{c}\text { Cycle } \\
\text { Count }\end{array}$ & $\begin{array}{c}\text { Speed } \\
(\mathrm{MHz})\end{array}$ & $\begin{array}{c}\text { Throughput } \\
(\mathrm{MBit} / \mathrm{s})\end{array}$ \\
\hline \hline CryptoManiac/4WC & 115 & 360 & 400 \\
PLD001 & 50 & 25 & 64 \\
\hline CRYPTONITE & 90 & 400 & 569 \\
\hline
\end{tabular}

Table 7.17: IDEA Performance Comparison

Also with IDEA CRYPTONITE shows good performance and outperforms its fully programmable competitor CryptoManiac by a factor of 1.42 ; even the IDEA-optimized PLD001 is outrun by a factor of 8.89 as shown in Table 7.17 and Figure 7.10.

\subsubsection{RC6 Performance}

With this algorithm, CryptoManiac is able to defeat CRYPTONITE using its complex functional units which make it possible to perform up to three sequential operations at once. It is not a matter of higher parallelism or 


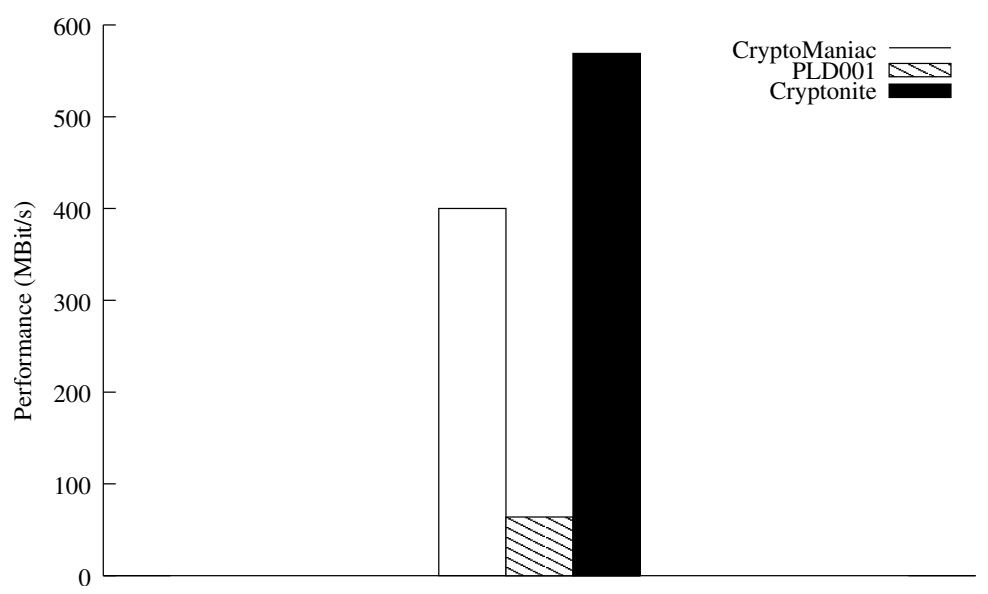

Figure 7.10: IDEA Performance Comparison

common data memory as Table 7.10 shows since the loop cycle count for RC6 is equal for all incarnations of the CryptoManiac architecture.

As shown in Table 7.18 and Figure 7.11 CRYPTONITE delivers about $29 \%$ less performance as CryptoManiac for RC6 in AES configuration.

\begin{tabular}{|l|ccc|}
\hline \multicolumn{1}{|c|}{ Architecture } & $\begin{array}{c}\text { Cycle } \\
\text { Count }\end{array}$ & $\begin{array}{c}\text { Speed } \\
(\mathrm{MHz})\end{array}$ & $\begin{array}{c}\text { Throughput } \\
(\mathrm{MBit} / \mathrm{s})\end{array}$ \\
\hline \hline CryptoManiac/4WC & 144 & 360 & 320 \\
\hline CRYPTONITE & 206 & 400 & 249 \\
\hline
\end{tabular}

Table 7.18: RC6 Performance Comparison

\subsubsection{MD5 Performance}

For MD5, CRYPTONITE again shows very good performance against the competing hardware cores and is able to deliver 1.08 to 6.77 times better throughput as the Hifn cores; as with previous examples it is again defeated by the specialized SecuCore solution which outperforms CRYPTONITE by a factor of 3.08 as shown in Table 7.19 and Figure 7.12. 


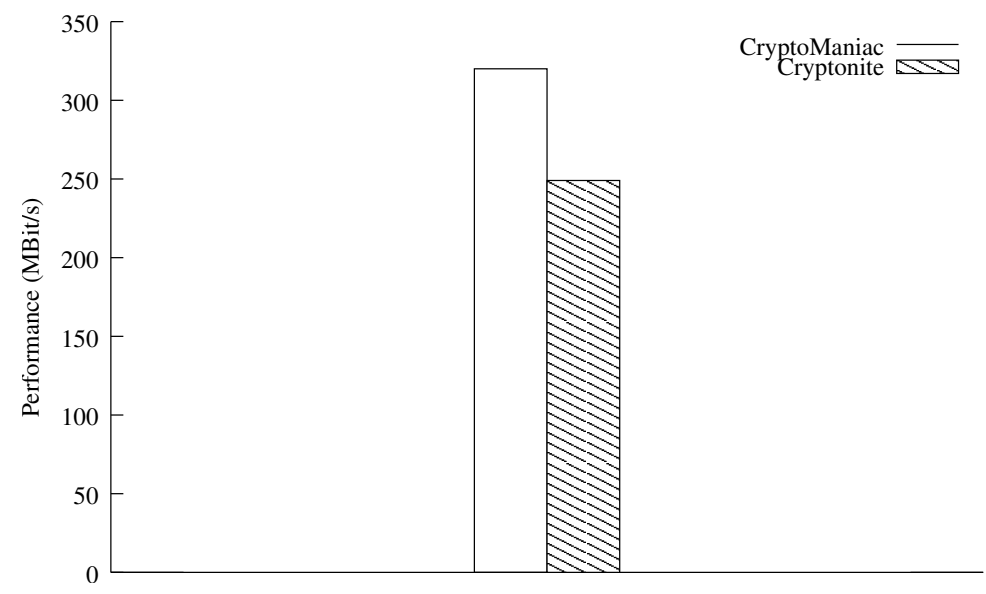

Figure 7.11: RC6 Performance Comparison

\begin{tabular}{|l|ccc|}
\hline Architecture & $\begin{array}{c}\text { Cycle } \\
\text { Count }\end{array}$ & $\begin{array}{c}\text { Speed } \\
\text { (MHz) }\end{array}$ & $\begin{array}{c}\text { Throughput } \\
\text { (MBit/s) }\end{array}$ \\
\hline \hline Hifn 7711 & 169 & 33 & 100 \\
Hifn 7751 & 176 & 33 & 96 \\
Hifn 7811 & 123 & 90 & 376 \\
Hifn 790x & 427 & 50 & 60 \\
SecuCore MD5 & 68 & 166 & 1250 \\
\hline CRYPTONITE & 504 & 400 & 406 \\
\hline
\end{tabular}

Table 7.19: MD5 Performance Comparison 


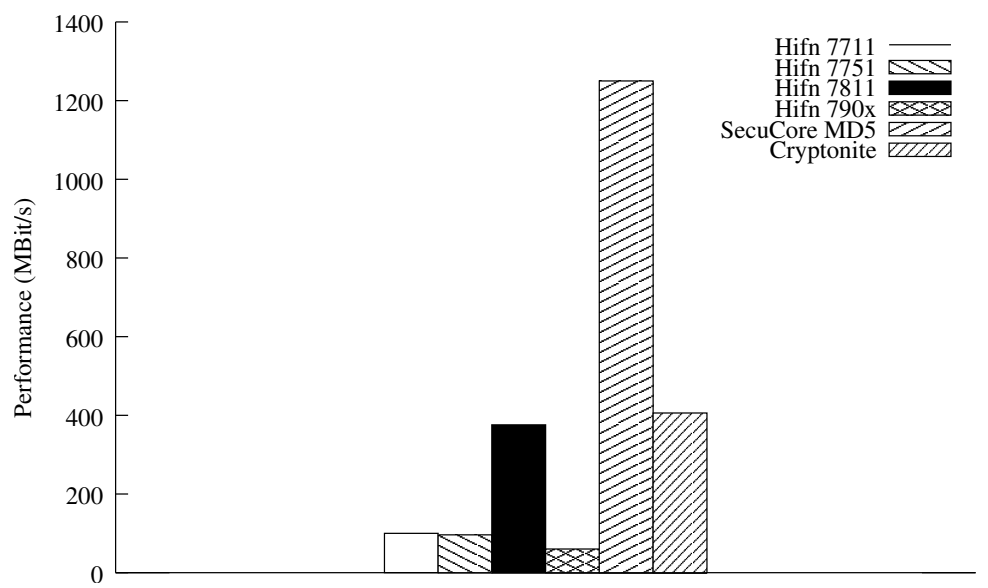

Figure 7.12: MD5 Performance Comparison

\subsubsection{SHA-1 Performance}

A similar picture is drawn by the SHA-1 performance comparison which shows 1.4 to 8.4 times better throughput for CRYPTONITE compared to the Hifn cores - but again the winning architecture is the dedicated hardware core from SecuCore defeating CRYPTONITE by a factor of 2.41 as shown in Table 7.20 and Figure 7.13.

\begin{tabular}{|l|ccc|}
\hline Architecture & $\begin{array}{c}\text { Cycle } \\
\text { Count }\end{array}$ & $\begin{array}{c}\text { Speed } \\
\text { (MHz) }\end{array}$ & $\begin{array}{c}\text { Throughput } \\
\text { (MBit/s) }\end{array}$ \\
\hline \hline Hifn 7711 & 201 & 33 & 84 \\
Hifn 7751 & 211 & 33 & 80 \\
Hifn 7811 & 153 & 90 & 301 \\
Hifn 790x & 512 & 50 & 50 \\
SecuCore SHA-1 & 84 & 166 & 1013 \\
\hline CRYPTONITE & 488 & 400 & 420 \\
\hline
\end{tabular}

Table 7.20: SHA-1 Performance Comparison 


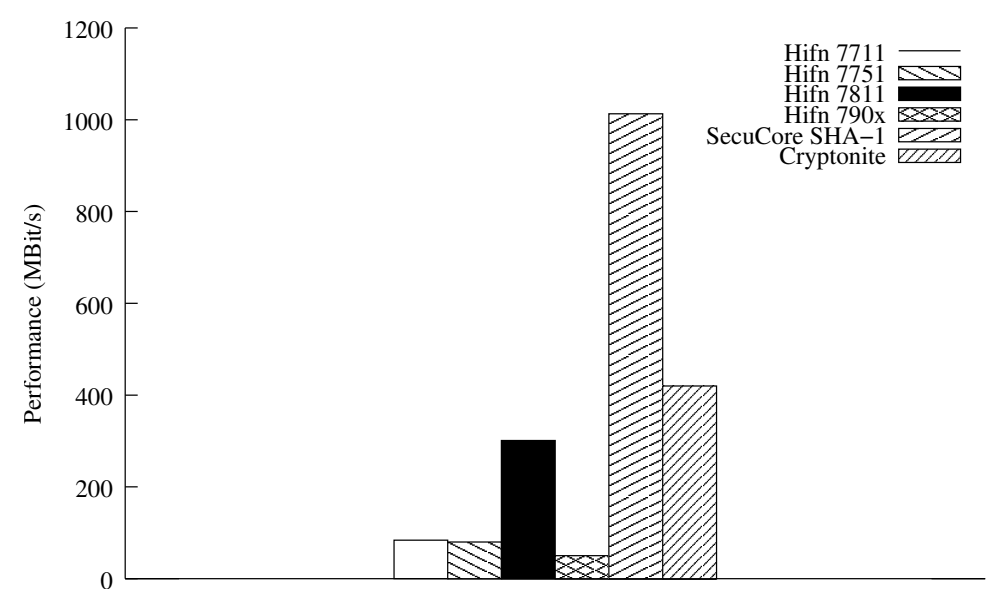

Figure 7.13: SHA-1 Performance Comparison

\subsection{Summary}

Within this chapter alternative crypto processors - both dedicated hardware and programmable solutions - were presented. In those cases where appropriate data was presented by the respective vendor, it was compared against the CRYPTONITE architecture; in some cases numbers had to be calculated from the given data and certain assumptions made. This was mentioned in the respective sections.

The comparison has clearly shown, that a modern programmable solution does not necessarily need to be slower than typical dedicated hardware solutions. In most cases CRYPTONITE outperformed the competing hardware cores by factors up to 5.42; even against high-performance cores like some of the Amphion solutions for AES-128/128 and all SecuCore cores CRYPTONITE was able to produce quite respectable results: The tradeoff for a fully programmable solution with as little special units or instructions as possible is a factor of less than 2.7 averaged over all cases where CRYPTONITE was defeated by hardware hardware solutions.

In addition the comparison especially with the CryptoManiac architecture has proven the algorithm analysis of Chapter 3 to be correct; also the architectural impact and proposals as elaborated in Chapter 4 resulting in the CRYPTONITE architecture presented in Chapter 5 were justified. The 
7. COMParison OF CRYPTONIte against Existing Solutions

CRYPTONITE architecture was proven to be efficient and competitive, both with programmable and dedicated hardware solutions. 


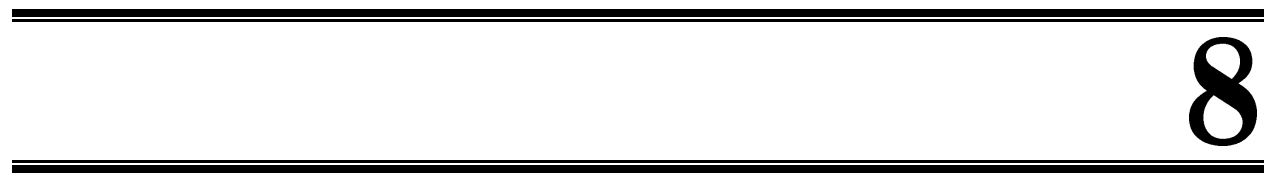

\section{Conclusion and Summary}

Within this work a novel, fully programmable processor architecture focused but not limited to cryptographic algorithms was presented.

In Chapter 1 the need for such an architecture was demonstrated together with some real-life examples. High-performance cryptography is pervading all fields of digital data distribution where broadcasted content has to be secured against unauthorized access. Of special interest is the field of high-bandwidth data distribution as necessary for all kinds of real-time data streams like video-on-demand (or, more generally, multimedia-on-demand) services, secured video conferencing or spanning of virtual private networks (VPNs) over existing network infrastructures.

Chapter 2 gives a general introduction into the field of cryptography together with basic definitions. It shows the difference between symmetric and asymmetric algorithms and explains typical operation modes of cryptographic algorithms.

For Chapter 3 a set of algorithms was selected and analyzed. The selection criteria was current and future proliferation; focus has clearly been put on the old crypto standard DES and the recently defined new standard, AES. Additional crypto and hash algorithms were added to investigate additional requirements by algorithms different from AES and DES.

This analysis' results and their impact on the proposed architecture have been listed in Chapter 4. Based on the algorithm analysis, needed operations were collected and weighted. The operations were distributed on functional units, namely Arithmetic Unit (AU) and XOR Unit (XU) both being part of the Arithmetic-Logical Unit (ALU). An accompaning Memory Unit (MU) 
enables the ALU to store its results to data memory or load previously stored data into registers of the register file. To exploit parallel structures found in certain algorithms this compound of ALU and MU called strand is doubled. An interlink mechanism provides a possibility to forward data and results between both strands.

Also, number and size of registers as well as supported data types were defined. For the analyzed algorithms a file of 4 registers fulfills the requirements if the already proposed interlink mechanism is extended by means of register value forwarding in addition to ALU results and memory data. As data types, 32-bit and 64-bit were defined with the possibility to individually access the two 32-bit halves of a 64-bit register allowing a 64-bit register to be used as two independent 32-bit registers. For data memory accesses this scheme is maintained and extended by the feature of so-called S-Box access as necessary for many crypto algorithms employing non-linear functions based on certain data tables; for an S-Box access the 64-bit of a memory cell are distributed evenly over eight 8-bit quantities each being addresses separately using a base address and an 8-bit offset provided through an similarly divided 64-bit index register. These eight 8-bit quantities are combined to a 64-bit result.

Furthermore, the need for special instructions was investigated. Due to its hardware-oriented nature DES clearly needs a specialized unit incorporating the necessary functions for the DES-specific operations, which are fixed bit permutations, compression and expansion operations together with round key generation. Also other algorithms could certainly benefit from specialized, monolithic instructions; the decision was made to only support AES with further instructions as needed for the fast AES/Rijndael implementation as provided by Agere Systems [45]. A reconfigurable modulomultiplier as needed by IDEA and RC6, for instance, are recommended but not necessary.

Based on this analysis, an architecture was constructed which is presented in Chapter 5. It consists of a control unit (CU) being responsible for command decoding and program flow control. For this reason, it consists of 16 so-called counter registers (CR) together with a small associated arithmetic unit enabling loop constructs and conditional branching; to also include conditions based on ALU results, the topmost four CRs mirror the sign and zero flags of the last ALU operation for each of the two ALUs. 
These ALUs divide into AU, XU and register file (RF) as described above with the ability to allow value and result forwarding between the two ALUs. Special operations as defined for fast AES operation are included into the AU. There is no direct connection from and to data memory; instead, registers of the RF can be loaded from data memory through the MU's Data Output Register (DOR); similarly, ALU results or register values can be stored to data memory through the MU's Data Input Register (DIR). Value transfer to registers is only possible through an ALU's A or X bus as shown in Figure 5.4 which means that during register load operations either AU or XU can not be used and have to be put in an idle state.

The memory unit (MU) is responsible for memory address generation and data transportation and thus divides into Address Generation Unit (AGU) and Data I/O Unit (DIO), the latter also containing the DES unit. The AGU does not only provide direct and S-Box addressing using but also indexed and modulo addressing; these more complex addressing modes are realized through a post-increment / post-modulo circuitry.

This architectural concept was tested using software emulators allowing test runs of algorithms implemented on the CRYPTONITE architecture. For selected algorithms the cycle count of certain algorithms is given in Chapter 4 which also covers the hardware implementation of the proposed architecture. Here, a number of functional units were described using VHDL, synthesized and fitted to the Xilinx [132] Virtex-IIpro FPGA family [133]. Based on these numbers together with other data provided by hardware core vendors speed estimations were done considering ASIC and custom chip layout implementations. This speed estimation forecasts a resulting speed range of 400 to $600 \mathrm{MHz}$ when using custom chip fabrication techniques.

In Chapter 7 the CRYPTONITE architecture is compared against existing crypto processor solutions; these include hardware cores being able to process only one algorithm, or a fixed set of algorithms, and programmable processors. All these solutions are listed together with their performance data. To allow independent comparison, absolute (MBit/s) and relative (Bits/Cycle) numbers were calculated based on the given data where not provided. The comparison against existing solutions has proven CRYPTONITE's high performance; even compared to hardware solutions it performs very well; the performance impact to be paid for programmabilty does usually not exceed a factor of 2.4 , which means that in worst case 
the proposed programmable solution still offers about $42 \%$ of a dedicated hardware solution.

In summary, this work has presented a novel programmable crypto processor architecture. This architecture was carefully designed with respect to high performance at moderate hardware demands. Running at $400 \mathrm{MHz}$ this architecture is able to outperform existing hardware solutions as well as comparable programmable solutions. Newer hardware solutions such as the SecuCore products of course show superior performance in their specific field; but even compared with these the programmable CRYPTONITE architecture is able to provide about $42 \%$ of the hardware core's performance. 


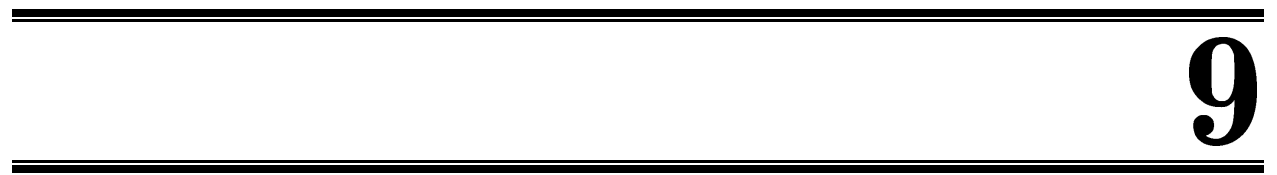

Future Work

The work presented within this document describes the core CRYPTONITE architecture which was developed with respect to speed and flexibility. Associated with this architecture there are further outstanding projects necessary for industrial use of the CRYPTONITE architecture which are listed in the following two sections. The third section covers possible areas of use.

\subsection{Software Development}

Currently, two simulator frameworks exist. One is based on Standard ML (SML), the other one is a cycle-based emulator written in C making it possible to write programs in an assembly language-like form. Both emulators, however, do not allow true native program development. In addition, the SML solution does not know about the CRYPTONITE architecture thus it neither knows about architectural restrictions nor does it support variableto-register mapping. Instead, the programmer needs to write programs architecturally mapped as if writing programs in assembly language.

The cycle-based emulator was designed to allow importing and running of CRYPTONITE binaries at a later development stage; until now it provides a framework for processing code in assembly language-like form, basically $\mathrm{C}$ files which call internal library routines mimicking assembly language commands. 


\subsubsection{CRyptonite Assembler}

For proper program development an assembler is essential and would be the first step in further software development. The assembler will contain source file parser, syntactical and semantical checks being able to detect syntax errors as well as breaches against the architectural rules, and finally code generation.

\subsubsection{C-Compiler for the CRYPTONITE Architecture}

A C compiler for the CRYPTONITE architecture is an ambitious task since it not only has to translate $\mathrm{C}$ to assembly language but also incorporate code analysis to exploit the maximum possible parallelism by assigning code as densely as possibly to the functional units. These tasks resemble the requirements for compilers for EPIC-style processors like the Intel Itanium [66]. Hence, it might be possible to adopt work done for the Trimaran citetrimaran project which originally targeted the HPL-PD architecture citehpl-pd but is flexible enough to be used for similar architectures as already shown through the Triceps [31] implementation, which mapped Trimaran to the ARM architecture.

\subsection{Hardware Development}

As of now, CRYPTONITE is just an architecture study. Only selected parts of the chip were realized and simulated using VHDL. This work describes the core architecture and only covers a simple interrupt-driven I/O mechanism; more sophisticated interface methods as needed for PCI-bus attachment or inter-processor communication have not yet been targeted. Further work definitely needs to cover these items to allow easy use of CRYPTONITE within existing computer systems.

\subsubsection{Single-Core CRYPTONITE}

A single-core CRYPTONITE would contain the CRYPTONITE core architecture together with an appropriate interface technology. This can be either a PCI interface to allow attachment of the CRYPTONITE to any PCI-based 
systems but also a simple synchronous (Intel x86-style) or asynchronous (Motorola 68k-style) bus interface to ease the use of CRYPTONITE with microcontrollers and non-PCI systems.

\subsubsection{Multi-Core CRYPTONITE}

Originally, CRYPTONITE was planned to be a SIMD multi-processor system consisting of multiple individual CRYPTONITE cores. Since quite a number of crypto algorithms is used in CBC mode, the SIMD approach is somewhat limited because it would allow only $n$ parallel streams - where $n$ is the number of cores - using the very same crypto algorithm.

A MIMD approach would be more flexible since it allows to process $n$ parallel streams using different algorithms. Such an architecture could contain internal load balancing to allow the assignment of incoming data streams to eventually idle cores or the take-over of lower priorized streams by higher priorized ones by detaching a running encryption from a core and reassigning this freed core.

Such a MIMD architecture contains several interesting challenges which are:

- Access of Program Data: Program data should not be present $n$ times individually for each processor core. Instead, it would be more sensible to employ some bootstrap mechanism which loads program routines as needed from a common program memory into individual program caches.

- Scheduling of Workload: To allow maximum throughput incoming data streams have to be assigned to idle cores; similarly, cores have to be freed after a stream has ended. The assignment procedure has also to cover loading of the correct program data into the individual core's program cache. Unnecessary loads have to be avoided by re-assigning already initialized cores where possible.

- Priorizing of Streams: Besides simple assignment of streams to idle cores, it must also be possible to priorize certain streams over others to allow some sort of quality-of-data service. This not only adds significant complexity to the scheduler but also brings up the problem of 
buffering of incoming information as well as inter-core communication and signalling.

\subsection{Use of CRYPTONITE}

Many dedicated hardware solutions exist but only very few programmable ones which brings up the question, where a programmable solution could be used and if that makes sense - especially, since naturally more sophisticated hardware cores show superior performance to any programmable solutions.

\subsubsection{Research \& Development}

$R \& D$ is the obvious candidate. Here a programmable solutions allows to create a very flexible experimental platform; algorithms and configurations can be checked without the need of re-designing hardware. A programmable high-performance solution will provide a realistic test environment.

\subsubsection{Conditional Access Systems}

The flexibility of a programmable high-performance solution makes it ideal for use in conditional access systems like TV set top boxes. In the past, several of these boxes were hacked in a way that the crypto algorithm was made public and pirate cards or completely independent decryption devices were developed.

In such cases, the access providers usually have to replace the existing conditional access module (CAM, also known as "Smart Card") which holds the decryption algorithm or even the complete set top box. Using a fully programmable high-performance crypto solution it would only require to update the crypto algorithm within the CAM.

\subsubsection{VPN Devices}

This is a field similar to the above. Usually, VPN devices make use of specific hardware solutions to enable high transfer speed. Once an algorithm becomes obsolete or unwanted because it is considered to be cryptographically weak, or if security demands require periodically change of the crypto 
algorithm, any device employing dedicated hardware solutions has to be exchanged or becomes obsolete. A programmable device would allow onthe-fly reconfiguration and save investment.

Following the R\&D approach, a programmable high-performance solution can be used for so-called market openers where already working, but less powerful (in terms of throughput) solutions are shipped to get later on updated by the final product which then uses dedicated hardware cores where sensible. 
9. FUtURE WORK 


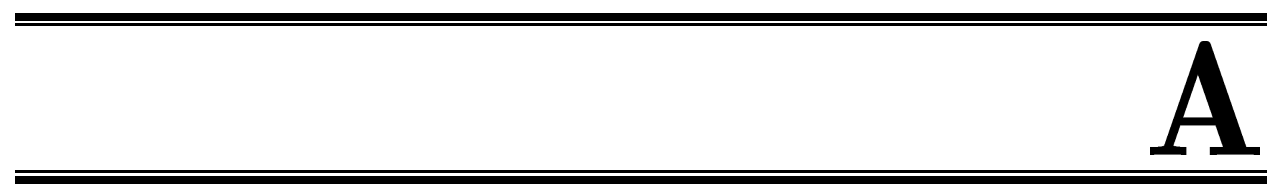

\section{Instruction Set}

The following chapter will present an overview over the CRYPTONITE instruction set. It does not cover the instruction word format which is explained in Appendix B.

\section{A.1 Immediate Values}

As mentioned in Chapter 5, CRYPTONITE basically supports two kinds of immediate values which are the global immediate values (GIVs) of 64-bit being used for ALU and MU register initialization and a 12-bit immediate used for counter register initialization and providing program addresses. The latter can be either used as destination address for branch instructions or immediate values for the MAU's local address registers. In addition, the MU instruction vector contains a 4-bit immediate field providing immediate index values or local address register values.

GIVs are only available through the CU's LDI instruction as shown in Table A.1 which changes the interpretation of the remaining instruction word from ALU/MU control to GIV transport. The 12-bit values are part of the "normal" instruction word and are transported through the address/immediate (A/I) field. See Appendix B.1 for a more detailed information on this topic. 


\begin{tabular}{|lll|}
\hline Instruction & Operands & Description \\
\hline LDI & $r e g_{1}, \# v a l_{1}, r e g_{2}, \# v a l_{2}$ & load 64-bit immediate value \\
& & val into register $r e g$ strands 1 \\
& and $2 ;$ reg can be either one \\
& of the ALU registers $r 0$ to r3 \\
& or the MU's data output reg- \\
& ister; Use of this instruction \\
& changes interpretation of the \\
& instruction word as explained \\
& in Appendix B. \\
\hline
\end{tabular}

Table A.1: Immediate Load Instruction (applies to Control Unit)

\section{A.2 Control Instructions}

These instructions apply to the Control Unit and are needed for program flow control as listed in Table A.2. The CU instructions divide into three groups (besides the special LDI instruction) which are counter register initialization ( $L D$ and $C L R$ ), branching (BRA, BNZ, BEZ, and DBNZ) plus the HOLD instruction which stops computation and puts CRYPTONITE on hold until an externally applied hold input signal is lowered. During this instruction, CRYPTONITE performs NOP operations on all slots while the program counter is not incremented. To enable and disable this kind of interrupt, EI and DI commands apply.

For the operands, reg refers to one of the multi-purpose registers employed within the control unit where $a d d r$ denotes a 12-bit address provided through the A/I within program memory. imm8 represents an 8-bit immediate also provided through the A/I field. This, of course, means that either an address or an immediate value can be provided at the same time.

\section{A.3 Memory Instructions}

Memory instructions fall into three classes which are register-to-memory, memory-to-register (A.3), and DES-specific operations (A.4). As men- 


\begin{tabular}{|c|c|c|}
\hline Instruction & Operands & Description \\
\hline $\mathrm{LD}$ & reg, \#imm8 & $\begin{array}{l}\text { load immediate value } i m m 8 \text { into } \\
\text { register } r e g\end{array}$ \\
\hline CLR & reg & short for LD $r e g, \# 0$ \\
\hline$\overline{\mathrm{DBNZ}}$ & $r e g, a d d r$ & $\begin{array}{l}\text { decrement register and branch to } \\
\text { address } a d d r \text { if the result is not } \\
\text { equal to zero }\end{array}$ \\
\hline BNZ & $a d d r, r e g$ & $\begin{array}{l}\text { conditional jump to address } \\
a d d r \text { if register reg is not zero }\end{array}$ \\
\hline BEZ & $a d d r, r e g$ & $\begin{array}{l}\text { conditional jump to address } \\
a d d r \text { if register reg equals zero }\end{array}$ \\
\hline BRA & $a d d r$ & $\begin{array}{l}\text { unconditional jump to address } \\
a d d r\end{array}$ \\
\hline EI & $\mathrm{n} / \mathrm{a}$ & enable external interrupt \\
\hline DI & $\mathrm{n} / \mathrm{a}$ & disable external interrupt \\
\hline HOLD & $\mathrm{n} / \mathrm{a}$ & $\begin{array}{l}\text { put CRYPTONITE into idle loop } \\
\text { until the signal applied to the } \\
\text { HOLD input pin is lowered }\end{array}$ \\
\hline $\mathrm{NOP}$ & $\mathrm{n} / \mathrm{a}$ & no operation \\
\hline
\end{tabular}

Table A.2: Control Instructions

tioned in Section 5.8.4 the DES unit is part of the MAU, therefore its instructions are listed in this section.

Here, lar refers to a local address register, namely $\operatorname{lr} 0$ to $\operatorname{lr} 7$ for memory-to-register operations and $1 \mathrm{w} 0$ to $1 \mathrm{w} 7$ for the reverse direction. $d s t$ and $s r c$ can be ALU registers ( $r 0$ to $r 3$, the data input/output registers dir and dor, or the link input ( $\operatorname{lnk}$ (applies only to $r 0$ ) together with size specification (.w or no specification for 64-bit, .h or . 1 for upper/lower 32-bit), e.g. XFR r0.h,r1.1 moves the low 32-bit of $r 1$ to the high 32-bit of $r 0$.

For index values or initialization data smaller than 16 , these values will be encoded into the memory unit's 4-bit immediate field. Otherwise, the MU's LAR instruction has to be used for loading bigger constants from the common $\mathrm{A} / \mathrm{I}$ field into the local address registers. 


\begin{tabular}{|c|c|c|}
\hline Instruction & Operands & Description \\
\hline LAR & lar, \#imm12 & $\begin{array}{l}\text { load local address register lar } \\
\text { with } 12 \text {-bit immediate value } \\
\text { imm } 12 \text { encoded in the in- } \\
\text { struction word's A/I section; } \\
\text { here } l a r \text { is a } 4 \text {-bit register ad- } \\
\text { dress where values } 0-7 \text { map to } \\
\operatorname{lr} 0 \text { to } \operatorname{lr} 7 \text { and } 8-15 \text { to } l \text { w } 0 \\
\text { to } l \mathrm{w} 7 \text {. }\end{array}$ \\
\hline $\mathrm{LDA}$ & $\operatorname{lar}[, i d x[+i d y / i m m]]$ & $\begin{array}{l}\text { load data from address in lar } \\
\text { into data input register; if in- } \\
\text { dex register } i d x \text { is present the } \\
\text { resulting address is addr }+ \\
{[i d x] \text { as mentioned in 5.8.1 }}\end{array}$ \\
\hline LBX & $l a r, i d x$ & $\begin{array}{l}\text { load S-box data from ad- } \\
\text { dress indexed by register idx } \\
\text { into destination register as de- } \\
\text { scribed in } 5.8 .1\end{array}$ \\
\hline STA & $\operatorname{lar}[, i d x[+i d y / i m m]]$ & $\begin{array}{l}\text { store data from data out- } \\
\text { put register to address in } \\
\text { lar; if index register } i d x \text { is } \\
\text { present the resulting address } \\
\text { is } a d d r+[i d x] \text { as mentioned } \\
\text { in } 5.8 .1\end{array}$ \\
\hline
\end{tabular}

Table A.3: Memory-to-Register and Register-to-Memory Instructions 
A.4. ARITHMETIC INSTRUCTIONS

\begin{tabular}{|c|c|c|}
\hline Instruction & Operands & Description \\
\hline$\overline{D E S \_D L}$ & lar & $\begin{array}{l}\text { load and input-permutate data from ad- } \\
\text { dress in lar into DES unit }\end{array}$ \\
\hline DES_KL & lar & $\begin{array}{l}\text { load and key-permutate key from address } \\
\text { in lar into DES unit }\end{array}$ \\
\hline DES_EX & $\mathrm{n} / \mathrm{a}$ & $\begin{array}{l}\text { perform first half of DES round including } \\
\text { S-Box lookup }\end{array}$ \\
\hline DES_PBEX & $\mathrm{n} / \mathrm{a}$ & $\begin{array}{l}\text { perform second half of current and first } \\
\text { half of next DES round including S-Box } \\
\text { lookup }\end{array}$ \\
\hline DES_PBWR & lar & $\begin{array}{l}\text { perform second half of DES round and } \\
\text { store output-permutated data to address in } \\
\text { lar }\end{array}$ \\
\hline
\end{tabular}

Table A.4: DES-specific Instructions

\section{A.4 Arithmetic Instructions}

Arithmetic instructions share the same generic form as defined by instr (dst, src1, src2) and follow the given definitions for $\operatorname{src}$ and $d s t$ operands with the extensions already mentioned in A.5. In addition, the ALU's accumulator ac can be used as an alternative source or destination operand. Also with the ALU it is only possible to assign a common size identifier to a sequence of destination registers.

The CRYPTONITE AU supports classes of operations which are Accumulator instructions, Boolean operations, simple arithmetic operations, complex arithmetic operations and special operations which are listed in Table A.5.

\section{A.5 XOR Instructions}

The XOR unit can be accessed in parallel to the arithmetic unit and provides only two instructions which are listed in Table A.6. The operand syntax follows the definitions given in A.3, although due to the nature of the XOR command up to six source operands, registers $r 0$ to $r 3$, the respective in- 


\begin{tabular}{|c|c|c|}
\hline Operation Class & Operation & Description \\
\hline Accumulator Instructions & STA & $\begin{array}{l}\text { load accumulator with register } \\
\text { value } \\
\text { store accumulator value to } \\
\text { register }\end{array}$ \\
\hline Boolean Operations & $\begin{array}{l}\text { AND } \\
\text { OR } \\
\text { XOR }\end{array}$ & $\begin{array}{l}\text { logical and } \\
\text { logical or } \\
\text { logical exclusive-or }\end{array}$ \\
\hline Shift Operations & $\begin{array}{l}\text { SHL } \\
\text { SHR } \\
\text { ROL } \\
\text { ROR }\end{array}$ & $\begin{array}{l}\text { logical left-shift } \\
\text { logical right-shift } \\
\text { rotate left } \\
\text { rotate right }\end{array}$ \\
\hline Arithmetics & $\begin{array}{l}\text { ADD } \\
\text { SUB } \\
\text { MUL16 } \\
\text { MUL32 }\end{array}$ & $\begin{array}{l}\text { addition w/o carry } \\
\text { subtraction w/o carry } \\
\bmod _{16} \text { integer multiplication } \\
\bmod _{32} \text { integer multiplication }\end{array}$ \\
\hline Special Operations & $\begin{array}{l}\text { SWAP } \\
\text { SWRT0 } \\
\text { SWRT1 } \\
\text { UPPER64 } \\
\text { LOWER64 } \\
\text { FOLDB32 } \\
\text { FOLDB } 64 \\
\text { FOLDW6 } 4\end{array}$ & $\begin{array}{l}\text { swap 32-bit halves of registers } \\
\text { swap and rotate halves individ- } \\
\text { ually by zero and } 8 \text { positions } \\
\text { swap and rotate halves individ- } \\
\text { ually by } 16 \text { and } 24 \text { positions } \\
\text { see Table } 5.3 \\
\text { see Table } 5.3 \\
\text { see Table } 5.5 \\
\text { see Table } 5.5 \\
\text { see Table } 5.5\end{array}$ \\
\hline miscellaneous & $\begin{array}{l}\text { NOP } \\
\text { XFR }\end{array}$ & $\begin{array}{l}\text { no operation (no operands) } \\
\text { transfer source to destina- } \\
\text { tion operand (only uses two } \\
\text { operands, } s r c \text { and } d s t \text { ) }\end{array}$ \\
\hline
\end{tabular}

Table A.5: ALU Instructions 
terlink input $l \mathrm{nk}$ and the value of the data output register dor are possible. The destination $d s t$ can be a sequence of up to 6 registers consisting of $r 0$ to $r 3$, dor and dir. This allows parallel storage of results into more than one register as needed for the fast AES implementation. To not overly increase the instruction word's size, parallel storage allows only one size identifier common to all destination registers.

\begin{tabular}{|c|c|c|}
\hline Instruction & Operands & Description \\
\hline NOP & $\mathrm{n} / \mathrm{a}$ & no operation \\
\hline $\mathrm{XFR}$ & $d s t, s r c$ & $\begin{array}{l}\text { transfer source to destination register by } \\
\text { routing the source value through the XOR } \\
\text { unit }\end{array}$ \\
\hline $\mathrm{XOR}$ & $d s t, s r c 1, s \imath$ & $\begin{array}{l}c 2[, \operatorname{src} 3[, \operatorname{src} 4[, \operatorname{src} 5[, \operatorname{src} 6]]]] \\
\text { logical XOR }\end{array}$ \\
\hline NEG & $d s t, s r c$ & binary negation \\
\hline
\end{tabular}

Table A.6: XOR Unit instructions 
A. Instruction Set

148 


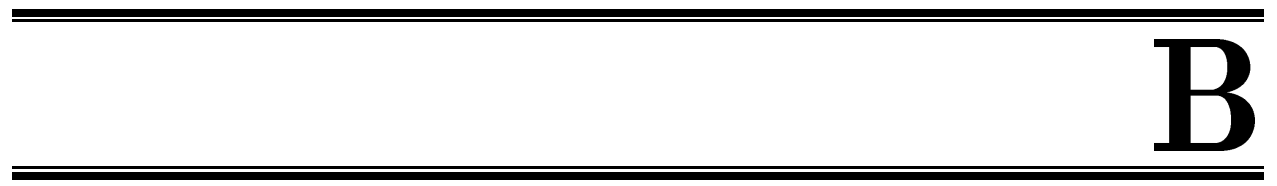

\section{Instruction Word Format}

CRYPTONITE is a VLIW-style machine with a 170-bit instruction word which divides into the the command unit (CU) section, and two of each sections for MAU, ALU and XOR unit (called a strand) which will be explained in the following sections. Global immediate values (GIV, 64-bit) are provided through a special instruction LDI which turns the strand-relevant bit fields into a 64-bit immediate value followed by a 3-bit register number. As the instruction word size is big enough, the LDI instruction can assign individual 64-bit values to each strand within one cycle for the sake of disabling any other operation.

\section{B.1 Instruction Word}

The instruction word contains the complete instruction bit stream controlling the internal units of CRYPTONITE. As presented in Chapter 5, CRYPTONITE divides into the sections for Control Unit (CU), two Arithmetic Units (AUs) which further divide into Arithmetic Logical Unit (ALU) and XOR-Unit (XU), plus two Memory Units (MUs) corresponding with the AUs, and finally the 12-bit address/immediate field which either holds a 12-bit program address or an 8-bit immediate value for counter registers.

Naturally, the instruction word format as shown in Table B.1 resembles this architecture and divides into smaller chunks each being assigned to an internal unit. For controlling settings common within one strand the ALU Common (AC) fields were added; as depicted in Table B.1 and already mentioned in Section 5.6, a special interpretation of the instruction word applies 
Normal Operation

\begin{tabular}{|c|c|c|c|c|c|c|c|c|c|}
\hline \multirow{3}{*}{$\mathbf{C U}$} & \multicolumn{4}{|c|}{ Strand \#1 } & \multicolumn{4}{|c|}{ Strand \#2 } & \multirow{3}{*}{$\mathrm{A} / \mathrm{I}$} \\
\hline & $\overline{\mathbf{A C}}$ & $\overline{\mathbf{A L U}}$ & $\mathbf{X U}$ & $\overline{\mathbf{M U}}$ & $\mathbf{A C}$ & $\overline{\mathbf{A L U}}$ & $\mathbf{X U}$ & MU & \\
\hline & \multicolumn{3}{|c|}{- AU \#1- } & & \multicolumn{3}{|c|}{$-\mathrm{AU} \# 2-$} & & \\
\hline
\end{tabular}

Immediate Load Instruction

\begin{tabular}{|l||l|l||l|l||l|}
\hline CU & Register & GIV & Register & GIV & n/a \\
\hline
\end{tabular}

Table B.1: CRYPTONITE Instruction Word Format

for the load of 64-bit immediate values. The following sections will give a detailed description of these chunks.

In several cases the size of employed registers, addressed by a 3-bit field as listed in Table B.2 has to be denoted by a 2-bit control value. This value maps to register sizes as shown in Table B.3. If ALU registers $r 0$ to $r 3$ are addressed, state $\mathrm{OO}_{2}$ on the input side means clearing the register; on the output side, the register is "muted" meaning that instead of the register's content the zero value is put on the referring output bus which is also true for the data output register. For the data input register (DIR) state $\mathrm{OO}_{2}$ represents the NOP value meaning that the DIR is left unchanged and not updated with an ALU result.

\begin{tabular}{|cc|l|}
\hline Register Address & rr $r$ \\
\hline \hline $\mathrm{rrr}=$ & 0xx & register $r 0$ to $r 3$ \\
& 100 & data output register \\
101 & data input register \\
110 & interlink \\
111 & reserved \\
\hline
\end{tabular}

Table B.2: Register Adresses

\section{B.2 Support of Immediate Values}

Since CRYPTONITE is a 64-bit architecture, providing support of immediate values requires 64 bits for the value itself plus 4 bits for the destination 


\section{B.3. Control Unit COMmand EnCODing}

\begin{tabular}{|rr|l|}
\hline \multicolumn{2}{|c|}{ Register Size } & SS \\
\hline \hline ss $=$ & 00 & mute/clear register (see Section B.1) \\
& 01 & address lower 32-bit half \\
& 10 & address upper 32-bit half \\
& 11 & address all 64-bit \\
\hline
\end{tabular}

Table B.3: Register Size Selection Values

register (registers $r 0$ to $r 3$, data input and output register of each strand). To not bloat the already wide instruction word for the CRYPTONITE architecture a different approach was taken to add support for immediate values. If the LDI (load immediate value) command is encountered, command vectors usually controlling ALU and associated MU will hold a 64-bit value and the register address. This is possible because the instruction vectors for ALU and MU are big enough to provide the necessary space to hold this information. Unused bits will be disregarded and should be padded by the assembler software with zero bits to ease future use.

\section{B.3 Control Unit Command Encoding}

As mentioned in Section 5.6 and further explained in Section A.2 the control unit supports 8 commands being responsible for program flow control. This also includes loading of counter registers with immediate values provided through the A/I field of the instruction word. Table B.4 shows the command encoding for CRYPTONITE's control unit. The CU contributes 8 bits to the instruction word.

\section{B.4 ALU-common Control Encoding}

The ALU contains some units which are shared either within the ALU (data input and output register of the corresponding MU) or between the two ALUs (interlink). Configuration is done through 6 bits per ALU as explained in Table B.5. 


\begin{tabular}{|rll|}
\hline CU Instruction Pattern & CCCC rrrr \\
\hline \hline CCCC & CU command code \\
0000 & NOP & no operation \\
0001 & HOLD & suspend and wait for Hold input signal to lower \\
0010 & EI & enable external interrupt \\
0011 & DI & disable external interrupt \\
0100 & LCR & load counter register denoted by $r r r r ~$ \\
0101 & LDI & load 64-bit immediate \\
$011 x$ & reserved \\
1000 & BRA & branch immediate \\
1001 & BEZ & branch if zero \\
1010 & BNZ & branch if not zero \\
1011 & DBNZ & decrement and branch if not zero \\
$11 x x$ & reserved \\
\hline register selection (cr0 to cr15)
\end{tabular}

Table B.4: Control Unit Instruction Format

\begin{tabular}{|c|c|}
\hline ALU Common Configuration Pattern & $1100 \mathrm{ii}$ \\
\hline Link Size (output size of opposit & ALU's register $r 0$ ) \\
\hline Data Output Register Size & \\
\hline i i Data Input Register Size & \\
\hline
\end{tabular}

Table B.5: ALU-common Configuration Pattern 


\section{B.5 XOR Unit Command Encoding}

The XOR unit is special in a way that it supports up to 6 source operands which are the four ALU registers $r 0$ to $r 3$, the interlink input and the current value of the MU's data output register. The command encoding is summed up in Table B.6. Each XU contributes 20 bits to the instruction word. The XFR instruction does not explicitly exist for this unit, instead the NOP operation applies together with appropriate source and destination register settings.

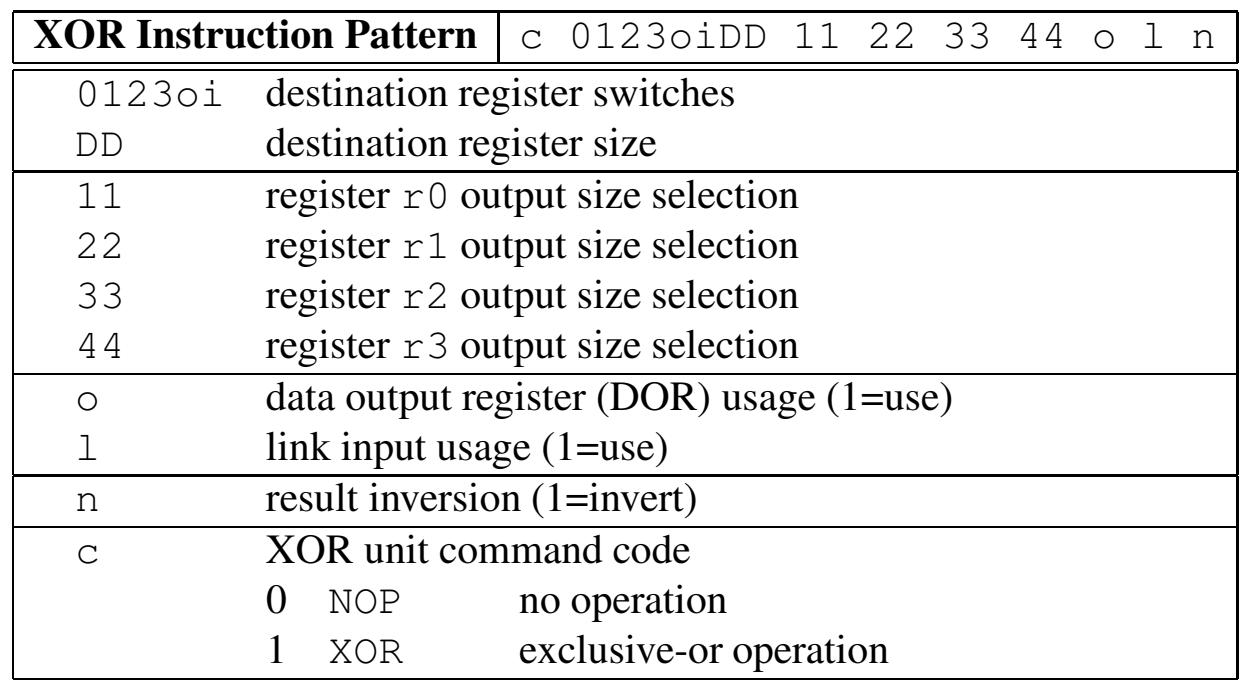

Table B.6: XOR Unit Instruction Format

\section{B.6 Arithmetic Unit Command Encoding}

This is the most complex unit of CRYPTONITE consisting of several subunits like the multiplication unit, the bitmux unit, and the configurable barrel shifter/rotator. Similarly to XU, also for the AU no dedicated XFR instruction exists; instead, register transfer is encoded into the appropriate register settings together with a NOP operation. The control word encoding is shown in Table B.7 and contributes 25 bits per ALU to the instruction word. 


\begin{tabular}{|c|c|c|c|}
\hline \multicolumn{2}{|c|}{ AU Instruction Pattern } & \multicolumn{2}{|c|}{ cCCCC r 01230iaDD sssSs tttTT } \\
\hline $\begin{array}{l}\text { 01230iaDD } \\
\text { ssssis } \\
\text { tttT }\end{array}$ & $\begin{array}{l}\text { destina } \\
\text { source } \\
\text { source }\end{array}$ & $\begin{array}{l}\text { ion register } \\
\neq 1 \text { register ar } \\
\$ 2 \text { register ar }\end{array}$ & $\begin{array}{l}\text { witches and size } \\
\text { d size } \\
\text { d size }\end{array}$ \\
\hline $\mathrm{CCCC}$ & $\begin{array}{l}\text { AU cor } \\
00000 \\
00001 \\
00010 \\
00011 \\
00100 \\
00101 \\
00110 \\
00111 \\
01000 \\
01001 \\
01010 \\
01011 \\
01100 \\
01101 \\
01110 \\
01111\end{array}$ & $\begin{array}{l}\text { Imand code } \\
\text { NOP } \\
\text { reserved } \\
\text { LDA } \\
\text { STA } \\
\text { AND } \\
\text { OR } \\
\text { XOR } \\
\text { SWAP } \\
\text { SWRT0 } \\
\text { SWRT1 } \\
\text { UPPER64 } \\
\text { LOWER64 } \\
\text { FOLDB32 } \\
\text { FOLDB64 } \\
\text { FOLDW64 } \\
\text { reserved }\end{array}$ & $\begin{array}{l}\text { no operation } \\
\text { load accumulator } \\
\text { store accumulator } \\
\text { logical and } \\
\text { logical or } \\
\text { logical xor } \\
\text { swap half-words } \\
\text { swap and rotate }(0 / 8) \\
\text { swap and rotate }(16 / 24) \\
\text { upper64 operation } \\
\text { lower64 operation } \\
\text { foldb32 operation } \\
\text { foldb64 operation } \\
\text { foldw64 operation }\end{array}$ \\
\hline $\mathrm{CCCC}$ & $\begin{array}{l}\text { AU cor } \\
10 r 00 \\
10 r 01 \\
\text { 10r10 } \\
\text { 10r11 } \\
\text { r: shift }\end{array}$ & $\begin{array}{l}\text { Imand code } \\
\text { SHL } \\
\text { SHR } \\
\text { ROL } \\
\text { ROR } \\
\text { r configurati } \\
0: 1 \times 64 \text {-bit } \\
\text { 1: } 2 \times 32-b i t \\
\text { MUL16 } \\
\text { MUL32 } \\
\text { ADD } \\
\text { SUB } \\
\text { reserved }\end{array}$ & $\begin{array}{l}\text { 16-bit } \bmod _{16} \text { multiplication } \\
\text { 32-bit } \bmod _{32} \text { multiplication } \\
\text { Addition } \\
\text { Subtraction }\end{array}$ \\
\hline
\end{tabular}

Table B.7: Arithmetic Unit Instruction Format 


\section{B.7 Memory Unit Command Encoding}

The Memory Unit provides 10 commands which cover register transfer, memory/register and register/memory transport, DES-specific operations and S-Box accesses as described in 5.8. Immediate values are supported in two ways, short individual 4-bit values encoded into the instruction format for providing index steps or table boundaries for modulo addressing, and 12-bit values for Local Address Register initialization and branch addresses as provided by the A/I field. The command encoding is shown in Table B.8. Each MU contributes 24 bits to the instruction word.

\begin{tabular}{|c|c|c|c|c|}
\hline \multicolumn{2}{|c|}{ MU Instruction Pattern } & o CCCC SS & $\mathrm{S}$ dDD xxx yyy $\mathrm{mmm}$ & iiii \\
\hline \multirow[t]{3}{*}{0} & \multicolumn{4}{|c|}{ " select ALU out data } \\
\hline & 0 & \multirow{2}{*}{\multicolumn{3}{|c|}{$\begin{array}{l}\text { route XOR output to Memory Unit } \\
\text { route AU output to Memory Unit }\end{array}$}} \\
\hline & 1 & & & \\
\hline \multirow[t]{13}{*}{$\mathrm{CCCC}$} & \multicolumn{4}{|c|}{ MU command code } \\
\hline & 0000 & NOP & no operation & \\
\hline & 0001 & LAR & $\begin{array}{l}\text { load locale address regis- } \\
\text { ter with immediate value }\end{array}$ & \\
\hline & 0010 & LDR & load data input register & \\
\hline & 0011 & STR & $\begin{array}{l}\text { store data output register } \\
\text { to memory }\end{array}$ & \\
\hline & $01 \mathrm{xx}$ & reserved & & \\
\hline & 1000 & DES_DL & DES data load & \\
\hline & 1001 & DES_KL & DES key load & \\
\hline & 1010 & DES_EX & DES expand operation & \\
\hline & 1011 & DES_PBEX & $\begin{array}{l}\text { DES expand \& P-box op- } \\
\text { eration }\end{array}$ & \\
\hline & 1100 & DES_PBWR & $\begin{array}{l}\text { DES P-box \& write-back } \\
\text { operation }\end{array}$ & \\
\hline & 1101 & reserved & & \\
\hline & $111 x$ & reserved & & \\
\hline
\end{tabular}

Table B.8: Memory Unit Instruction Format

(continued on next page) 


\begin{tabular}{|c|c|c|c|}
\hline \multicolumn{2}{|c|}{ MU Instruction Pattern } & O $\quad$ CCCC $\quad$ SSS dDD & xxx yyy mmm iiii \\
\hline \multirow[t]{3}{*}{ SSS } & \\
\hline & \multicolumn{3}{|c|}{$\begin{array}{l}\text { source operand } S \text { and size } S S \\
0 \quad \text { Data Input Register }\end{array}$} \\
\hline & 1 & \multicolumn{2}{|l|}{ Data Output Register } \\
\hline $\mathrm{dDD}$ & \multicolumn{3}{|c|}{ destination operand $\mathrm{d}$ and size $\mathrm{DD}$} \\
\hline $\mathrm{x} x \mathrm{x}$ & \multicolumn{3}{|l|}{ index register } \\
\hline YYY & \multicolumn{3}{|l|}{ increment register } \\
\hline $\mathrm{mmm}$ & \multicolumn{3}{|l|}{ addressing mode } \\
\hline & \multicolumn{2}{|l|}{000} & plain \\
\hline & 001 & $i d x$ & indexed \\
\hline & 010 & $i d x+i d y$ & indexed $\mathrm{w} /$ register postincrement \\
\hline & 011 & $i d x+i m m$ & $\begin{array}{l}\text { indexed w/ immediate postincre- } \\
\text { ment }\end{array}$ \\
\hline & 100 & & S-Box access \\
\hline & 101 & & DES-based S-Box access \\
\hline & 110 & $(i d x+i d y) \% i m m$ & $\begin{array}{l}\text { indexed } \mathrm{w} / \text { register postincrement } \\
\text { and immediate modulo }\end{array}$ \\
\hline & 111 & $(i d x+i m m) \% i d x$ & $\begin{array}{l}\text { indexed w/ immediate postincre- } \\
\text { ment and register modulo }\end{array}$ \\
\hline
\end{tabular}

Table B.8: Memory Unit Instruction Format (continued) 


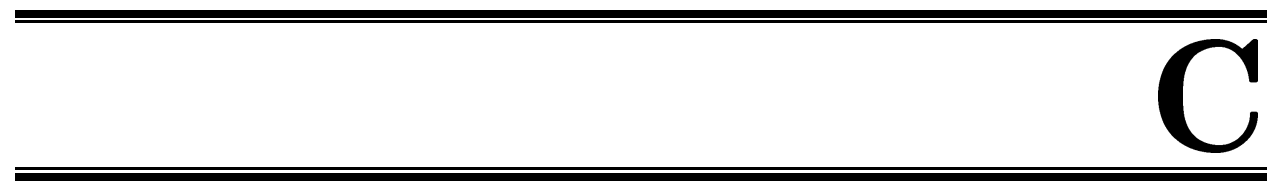

\section{Proposed Assembly Language Format}

Naturally, the assembly language format very much resembles the target architecture. Since CRYPTONITE is a parallel VLIW-style architecture dividing into Control Unit and two individual strands consisting of ALU and associated Memory Unit this chapter will work out definitions and conventions regarding the CRYPTONITE assembly language format.

\section{C.1 Register Naming Convention}

CRYPTONITE consists of two identical strands, each containing four ALU registers and 16 local address registers distributing on 8 local read and 8 local write registers. In addition, 16 counter registers exist which are strandindependent. To linearize register naming, the convention listed in Table C.1 applies.

\section{C.2 Assembly Language Format}

Following this register naming convention, the assembly language format does not necessarily need to mimic the CRYPTONITE architecture as shown in Table C.2. However, doing so will add clear structure to the code and ease both, program development and debugging since the programmer can easily keep track on used and spare units and the data flow.

Since due to the comparatively small sizes of typical crypto algorithms it is very likely that CRYPTONITE will be programmed in plain assembly 


\begin{tabular}{|l|c|}
\hline \multicolumn{1}{|c|}{ Register Type } & Register Name \\
\hline \hline Counter Register & $\mathrm{cr} 0-\mathrm{cr} 11$ \\
ALU \#1 Zero Flag & $\mathrm{cf} 0$ or $\mathrm{cr} 12$ \\
ALU \#1 Carry Flag & $\mathrm{zf} 0$ or $\mathrm{cr} 13$ \\
ALU \#2 Zero Flag & $\mathrm{cf} 1$ or $\mathrm{cr} 14$ \\
ALU \#2 Carry Flag & $\mathrm{zf} 1$ or $\mathrm{cr} 15$ \\
\hline
\end{tabular}

\begin{tabular}{|c|c|c|}
\hline Register Type & Strand \#1 & Strand \#2 \\
\hline ALU Register & $r 0-r 3$ & $r 4-r 7$ \\
\hline LAR/read & $\operatorname{lr} 0-\operatorname{lr} 7$ & $\operatorname{lr} 8-\operatorname{lr} 15$ \\
\hline LAR/write & $1 \mathrm{w} 0-1 \mathrm{w} 7$ & 1w8-1w15 \\
\hline
\end{tabular}

Table C.1: Register Naming Conventions

language. For this reason, human programmability becomes an issue. Programmers, however, usually dislike following strict schemes as shown in C.2 (although doing so would likely prevent quite a number of programming issues) but rather address units directly. For this reason, a CRYPTONITE mnemonic should follow the notation given in Table C.3.

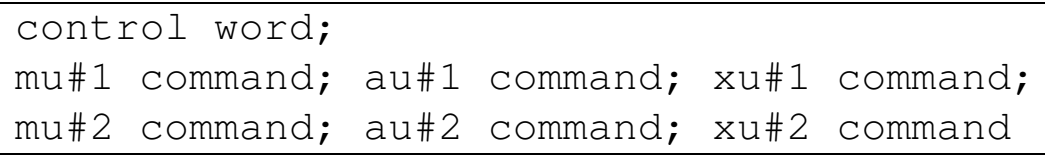

Table C.2: VLIW-style Assembly Instruction

As there is only one Control Unit within CRYPTONITE, there is no need to add any unit identifier to CU-related commands, these apply only for strand-relevant commands which are A for ALU, M for MU, and X for XU. These identifiers make it possible to maintain same names for equal operations since the assignment to units is done through the unit identifier which eases memorizing the CRYPTONITE commands: For example, the difference between an ALU-based or an XU-based XOR operation would simply be XOR. A as opposed to XOR. X. The assignment of commands to strands is usually done through the register numbering. In some cases, namely the DES_EX and DES_PBEX instructions, no parameters are assigned. Here, the 


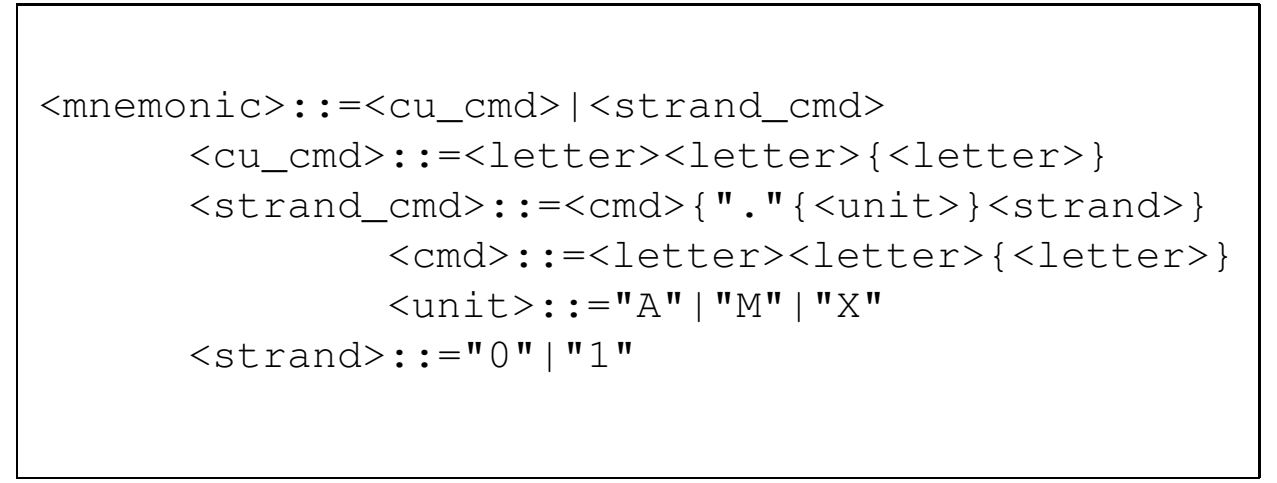

Table C.3: BNF Notation of a CRYPTONITE Mnemonic

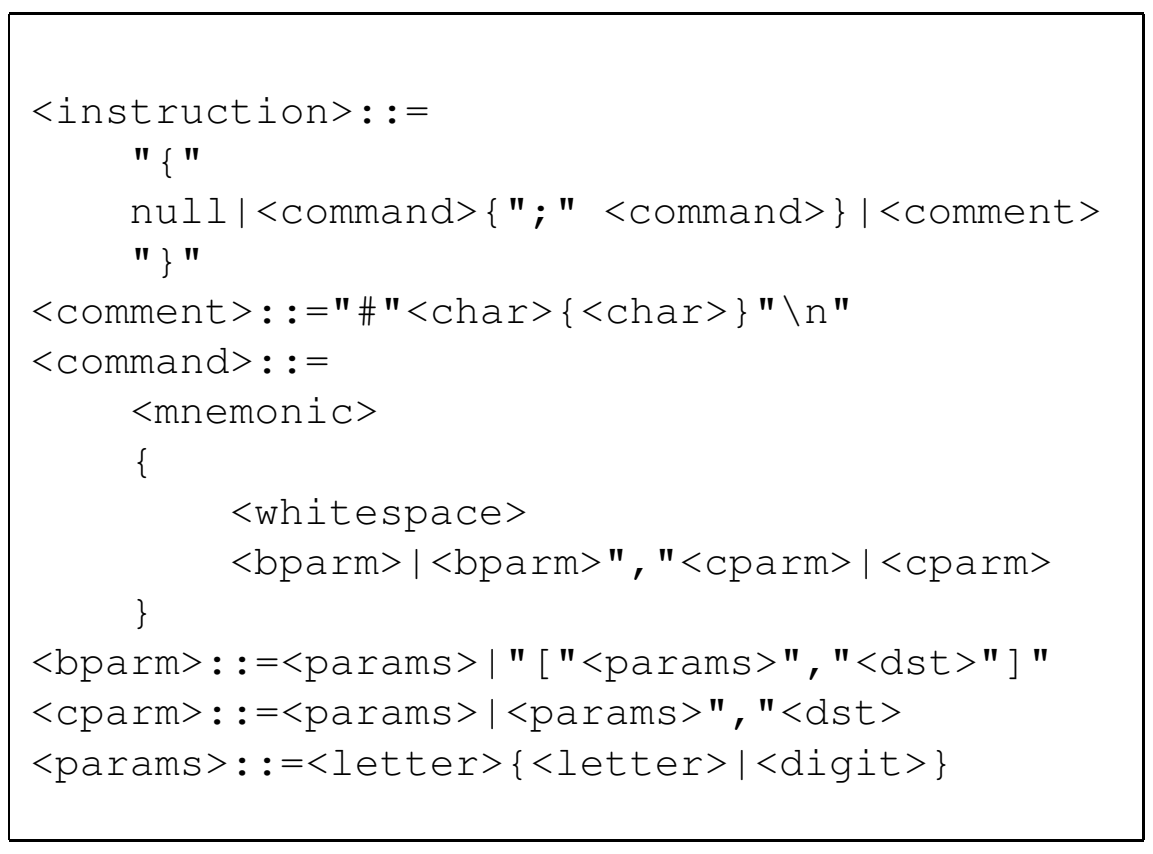

Table C.4: BNF Notation of a CRYPTONITE Instruction 


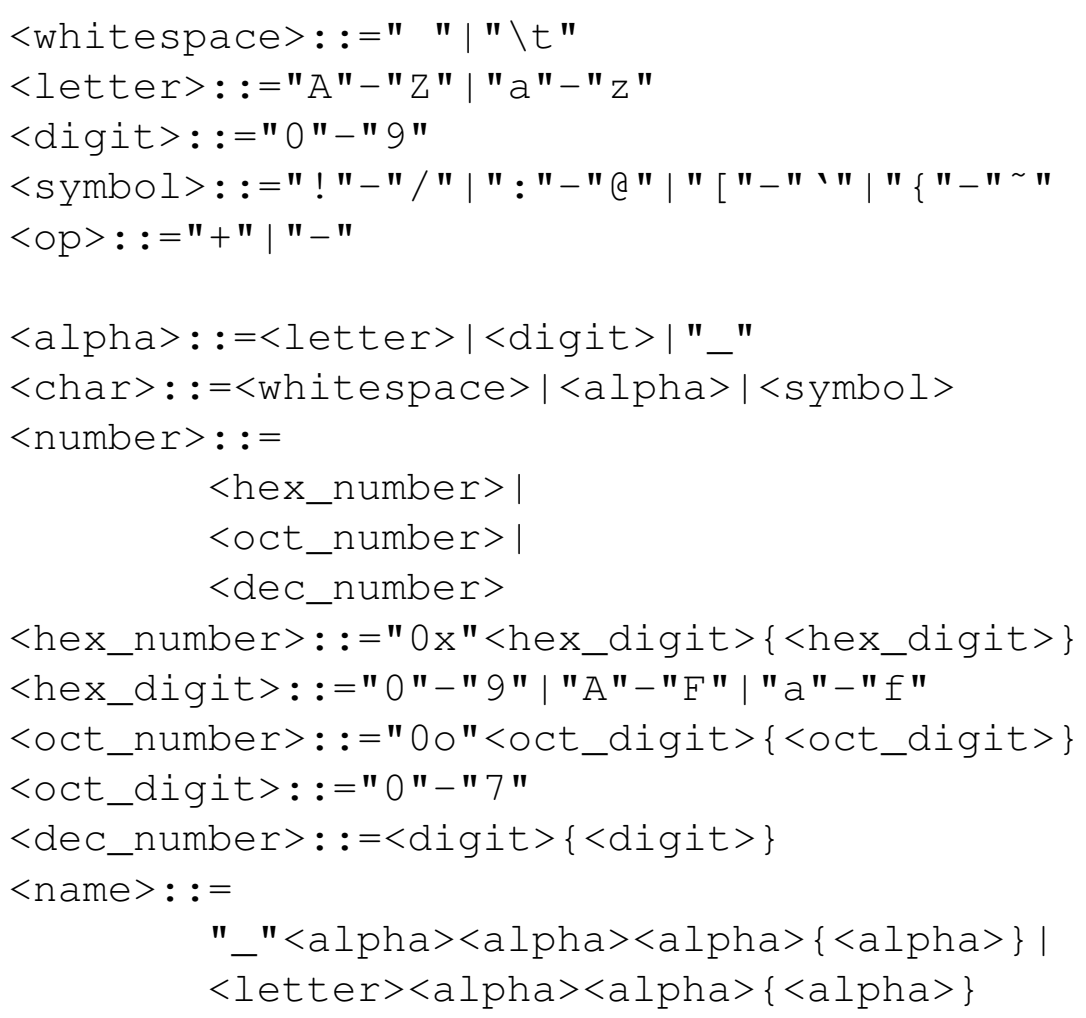

Table C.5: BNF Notation of Primitives and Composita 
strand is denoted by the respective number, . 0 or .1 . If a command can be assigned to a unit by name - which is usually the case - the unit identifier can be omitted. Similarly, the strand number can be omitted if the assignment can be made based on the register number.

To not force the programmer to fill up unused slots with unnecessary NOP instructions, bundles of commands which should be assigned to the CRYPTONITE units within the same cycles are enclosed in curly brackets. This leads to the definition of a complete assembly instruction shown in Table C.4. Together with the unit/strand assignment rules, writing NOP instruction are completely avoided. Instead, the assembler initializes each instruction word with NOP instructions for all units and replaces these with the commands found within each assembly language instruction.

CRYPTONITE supports branch instructions. For this reason, the assembly language also needs to support labels as branch targets. The definition for labels is given in Table C.6. Similarly, an assembly language should support constant definitions which together with CRYPTONITE is especially useful for assigning local memory addresses to more descriptive names. The definition for such constant assignments is also given in Table C.6.

Finally, it must been noted that certain ALU commands allow multiple destinations. In such cases, the comma-separated list of destination operands has to be enclosed in square brackets as shown in Table C.4.

$<$ label_decl $>:=<$ name $>$ ":"

$<$ const_decl $>::=$

$<$ name $>$

$<$ whitespace $>\{<$ whitespace $>\}$

$<e q u>$

$<$ whitespace $>\{<$ whitespace $>$ \}

$<$ number $>$ |

$<$ name $>\{\langle o p\rangle<$ number $\rangle\}$

$<$ equ $>:$ : ="EQU" | "equ"

Table C.6: BNF Notation of Labels and Constant Declarations 


\section{C.3 Programming Examples}

This section will now contain some basic programming examples followed by a complete algorithm implementation.

\section{C.3.1 Example 1: Initialization}

This example starts with a HOLD command which puts CRYPTONITE on hold and waits until external initialization (signalled through the level of the HOLD input line) is finished. Following this instruction, counter register $\operatorname{cr} 0$ and ALU registers $r 0$ (strand \#1) and $r 4$ (strand \#2) are cleared. Local address registers $\operatorname{lr} 0$ (strand \#1) and $\operatorname{lr} 8$ (strand \#2) for reading are initialized to memory location $0 \times 0000$.

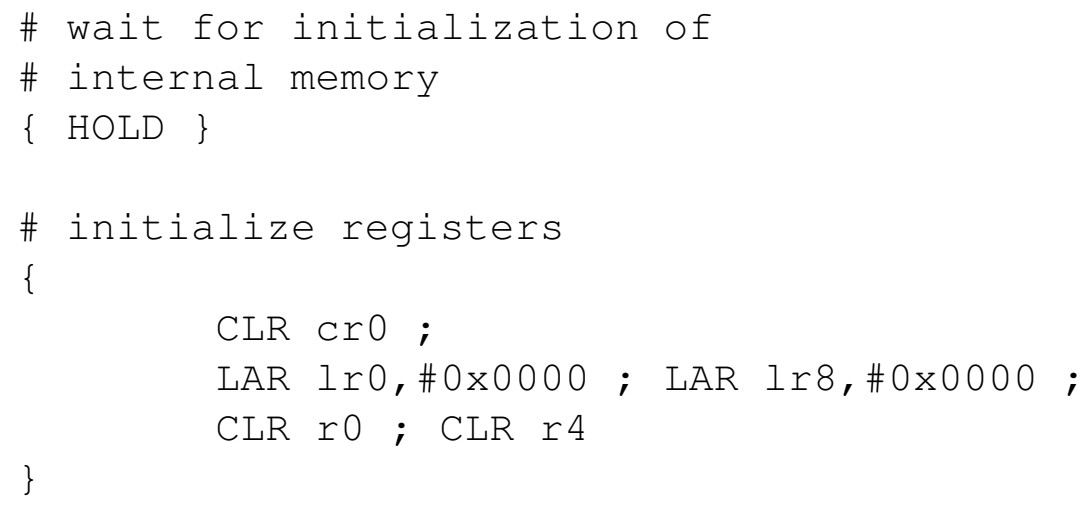

\section{C.3.2 Example 2: Update key \& data, return processed data}

For crypto algorithms it is vital to return processed data and fetch in new input data such as plain text and key updates. This example demonstrates how to achieve this with CRYPTONITE. The main crypto algorithm is embedded within DI and EI instructions to disallow interrupts during the encryption process which makes sure that updates will only happen after the current data block is processed. Since this is mainly a control flow example, it does not contain any computation relevant parts. In real life, it will be most likely possible to embed all these control instructions which will save cycles and program size. 
main:

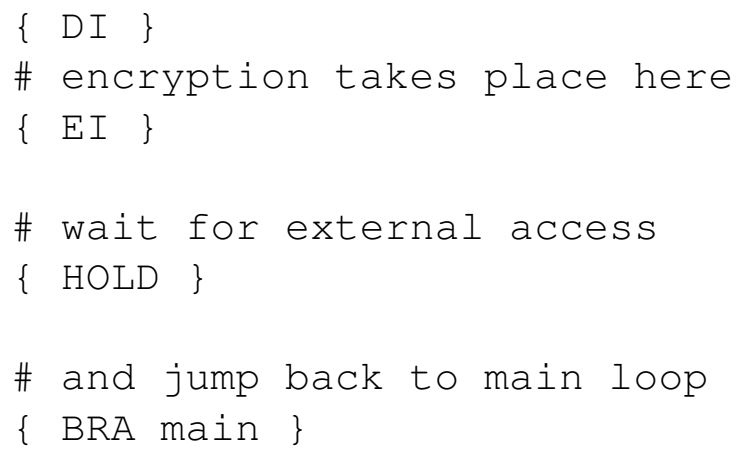

\section{C.3.3 Example 3: DES Implementation}

This DES implementation will now give an example for a complete algorithm implementation. Although this algorithm does not make use of all the CRYPTONITE's features it will give an easy to understand example for using the CRYPTONITE assembly language. It also shows the need for partially unrolling loops to circumvent pipelining issues together with branch instructions.

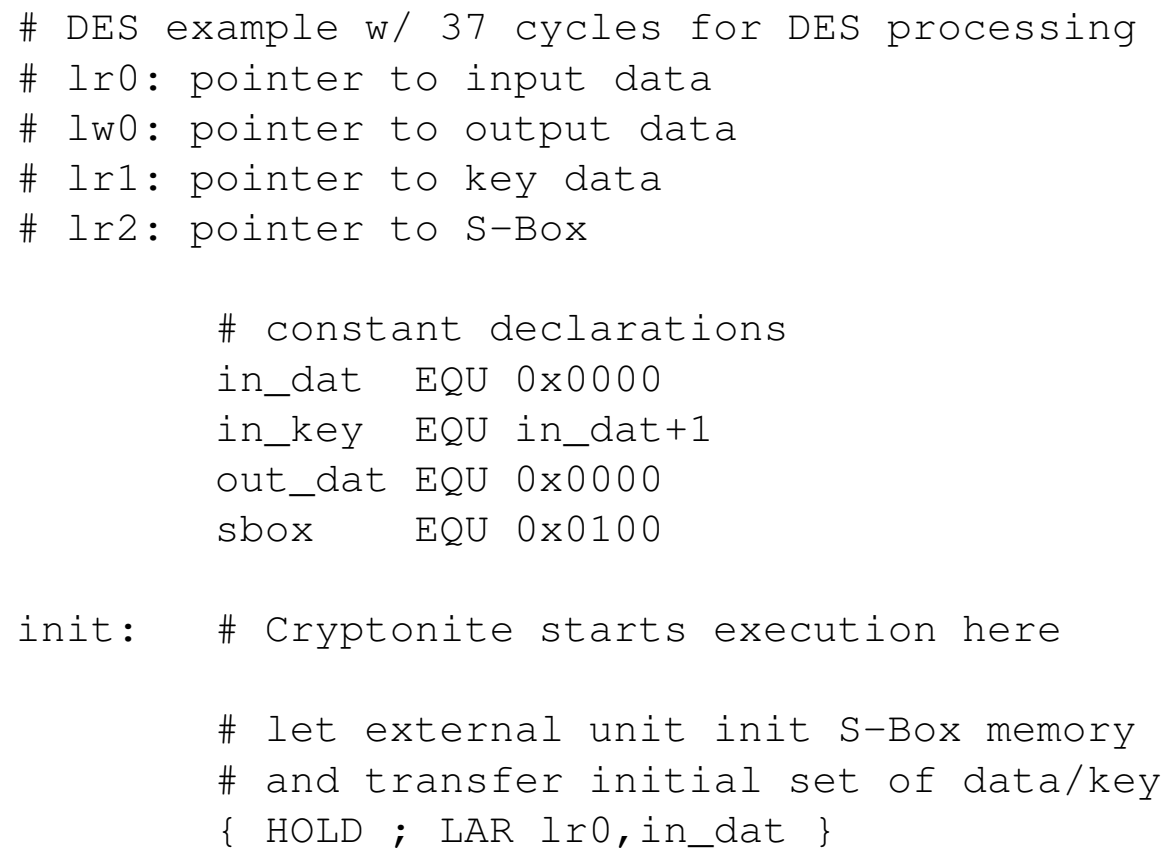




\section{Proposed Assembly Language Format}

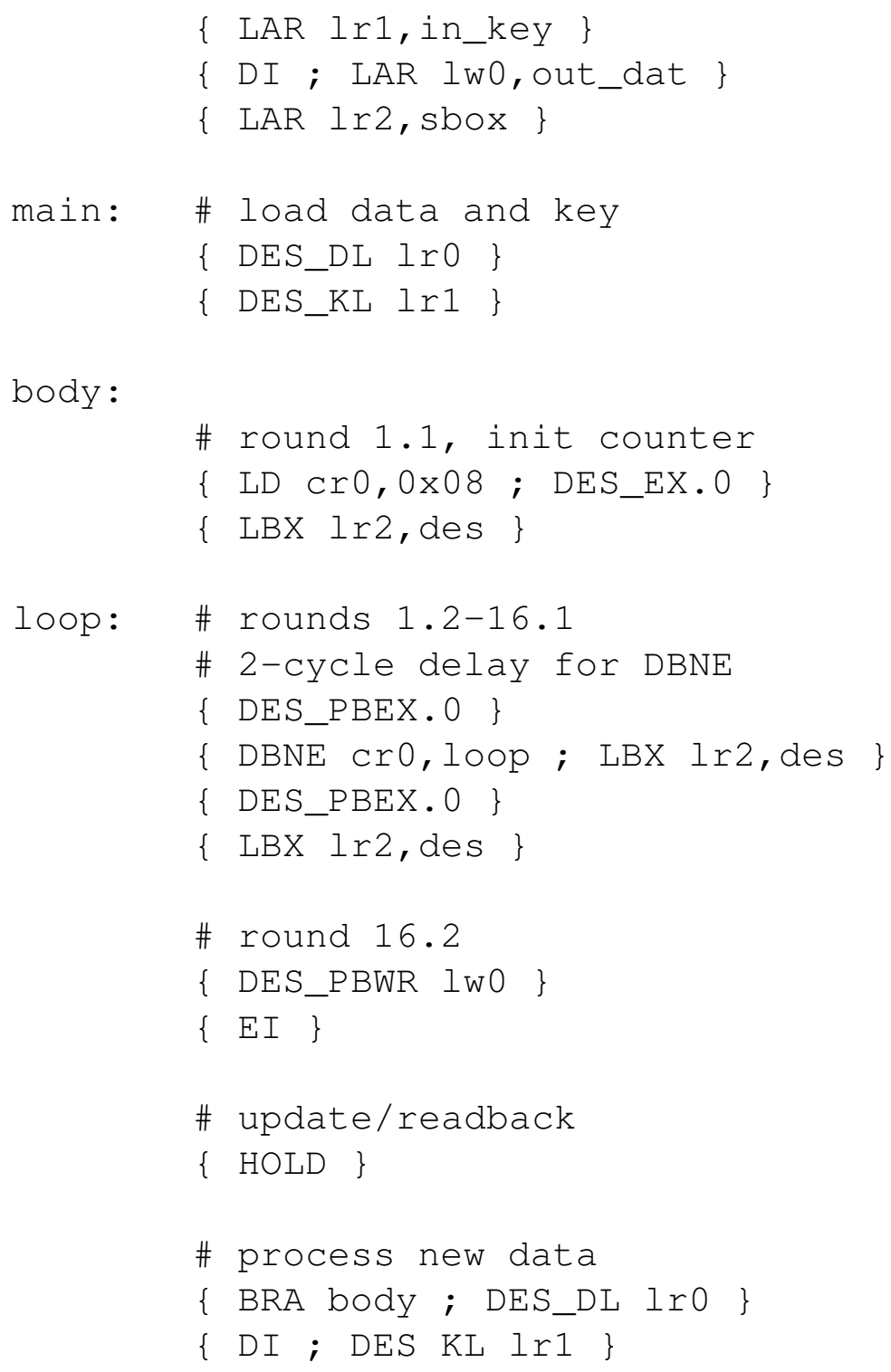

body :

\# round 1.1, init counter

\{ LD cr0,0x08; DES_EX.0 \}

\{ LBX 1r2, des \}

loop: \# rounds 1.2-16.1

\# 2-cycle delay for DBNE

$\{$ DES_PBEX.0 \}

\{ DBNE cr0, loop ; LBX lr2, des \}

$\{$ DES_PBEX.0 \}

$\{$ LBX $1 r 2$, des $\}$

\# round 16.2

\{ DES_PBWR lw0 \}

$\{\mathrm{EI}\}$

\# update/readback

\{ HOLD \}

\# process new data

\{ BRA body ; DES_DL IrO \}

$\{\mathrm{DI} ; \mathrm{DES} \mathrm{KL} \operatorname{lr} 1\}$ 


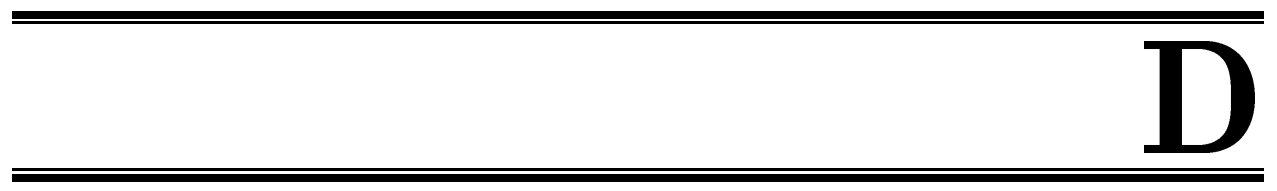

\section{Glossary}

3DES

AES

ALU

ASIC

Asymmetric

Cryptography

AU

Barrel Shifter

CPLD
Also known as Triple-DES; consists of three sequentially performed iterations of $16 \triangleright$ DES rounds. Can be either used with two keys where iterations one and three use the first and iteration two the second key, or with individual keys per iteration.

Advanced Encryption Standard; new $>$ NIST crypto standard from 2001. The AES standardization process was an open competition of many algorithms.

Arithmetic Logical Unit; a CRYPTONITE ALU contains $>\mathrm{AU}$ and $>\mathrm{XU}$

Application Specific Integrated Circuit; used as a cost-friendly alternative to fully custom chip design

Cryptographic method using a pair of keys, one for encryption $\left(K_{1}\right)$ and one for decryption $\left(K_{2}\right)$ so that the formula $M=D_{K_{2}}\left(C_{K_{1}}(M)\right)$ is valid with $M$ being the message, $C$ the encryption and $D$ the decryption function.

Arithmetic Unit

Circuitry being able to perform bit shifts of arbitrary size within one cycle

Complex Programmable Logic Device; $>$ EEPROMbased programmable logic device with complexities between 32 and 512 macrocells, typically used for glue logic or simple control devices 


\begin{tabular}{|c|c|}
\hline $\mathbf{C U}$ & Control Unit \\
\hline DES & $\begin{array}{l}\text { Data Encryption Standard; former }>\text { NIST crypto } \\
\text { standard from } 1977\end{array}$ \\
\hline DRM & $\begin{array}{l}\text { Digital Rights Management; (mostly cryptographic) } \\
\text { methods to maintain author rights for digital media }\end{array}$ \\
\hline EAU & $\begin{array}{l}\text { External Access Unit; interface between CRYP- } \\
\text { TONITE core and external devices }\end{array}$ \\
\hline EEPROM & $\begin{array}{l}\text { Electrically Erasable Programmable Read-Only } \\
\text { Memory }\end{array}$ \\
\hline FPGA & $\begin{array}{l}\text { Field Programmable Gate Array; usually } \rightarrow \text { SRAM- } \\
\text { based programmable logic device allowing to be } \\
\text { loaded with fairly complex logic, often used for pro- } \\
\text { totyping } \longrightarrow \text { ASICs }\end{array}$ \\
\hline IDEA & International Data Encryption Algorithm \\
\hline Key Generation & $\begin{array}{l}\text { Process of generating big prime numbers as }>\text { public } \\
\text { and }>\text { private key for }>\text { asymmetric cryptography; not } \\
\text { to be mixed up with }>\text { round key generation }\end{array}$ \\
\hline $\begin{array}{l}\text { Logical Element } \\
\text { (LE) }\end{array}$ & $\begin{array}{l}\text { Similar to macrocell but usually refers to }>\text { ASICs or } \\
- \text { FPGAs }\end{array}$ \\
\hline Loop & see $>$ Loop \\
\hline Macrocell & $\begin{array}{l}\text { Building block within }>\text { CPLDs holding a config- } \\
\text { urable storage cell (flip flop) with input and output } \\
\text { switch matrices }\end{array}$ \\
\hline MD4 & $\begin{array}{l}\text { Message Digest \#4, Hash Algorithm producing a 512- } \\
\text { bit hash }\end{array}$ \\
\hline MD5 & $\begin{array}{l}\text { Message Digest \#5, Hash Algorithm producing a 512- } \\
\text { bit hash; successor to }>\text { MD4 }\end{array}$ \\
\hline NIST & National Institute of Standards and Technology \\
\hline Private Key & Secret key for asymmetric encryption and decryption \\
\hline Public Key & $\begin{array}{l}\text { Publicly shared key for asymmetric encryption and } \\
\text { decryption }\end{array}$ \\
\hline RC4 & Rivest Cipher \#4, Cipher Algorithm \\
\hline RC5 & $\begin{array}{l}\text { Rivest Cipher \#5, Cipher Algorithm; successor to } \\
-\mathrm{RC} 4\end{array}$ \\
\hline RC6 & $\begin{array}{l}\text { Rivest Cipher \#6, Cipher Algorithm; successor to } \\
\rightarrow \text { RC5 and competitor in the } \rightarrow \text { AES selection }\end{array}$ \\
\hline
\end{tabular}


Rijndael Cipher Algorithm named after the inventors V. Rijmen and J. Daemen; winner of the AES competition

Round Key Gen- Method of generating individual keys for each eneration cryption (or decryption) round of an algorithm; these keys are based on a provided cipher (or decipher) key.

SHA Secure Hash Algorithm

SHA-1

SHA-256

SHA-384 Secure Hash Algorithm \#1 producing 160-bit hash; first hash algorithm to be standardized by $>$ NIST

Secure Hash Algorithm producing a 256-bit hash value

SHA-512

Secure Hash Algorithm producing a 384-bit hash value

Secure Hash Algorithm producing a 512-bit hash value

Slice $\quad$ Xilinx-specific term for logical elements within their Virtex family of $>$ FPGAs

Strand Within this document, the term strand is used to describe (mainly) data independent branches of a data dependency graph. The more pictural term thread is omitted to avoid confusion since in computer science literature this term is commonly used as a synonym for lightweighted processes. The concept of strands is also mapped to the architectural description where one strand is a compound of functional units ( ALU and associated $\longrightarrow$ MU) being able to process such data independent branches.

SRAM Static Random-Access Memory

Symmetric Cryptographic method using the same key $K$ for enCryptography cryption and decryption so that the formula $M=$ $D_{K}\left(C_{K}(M)\right)$ is valid with $\mathrm{M}$ being the message, $\mathrm{C}$ the encryption and $\mathrm{D}$ the decryption function. In addition, $C$ and $D$ can be identical and differ only in round key calculation.

Synopsys Both, vendor (Synopsys, Inc.) of an integrated development software for hardware design, and the name of the software itself 


$\begin{array}{ll}\text { Triple-DES } & \text { see 3DES } \\ \text { Tunnel } & \text { Virtual direct connection between two network nodes } \\ & \text { using }>\text { VPN mechanisms } \\ \text { VHDL } & \text { VHSIC Hardware Description Language } \\ \text { VHSIC } & \text { Very High Speed Integrated Circuit } \\ \text { VLIW } & \text { Very Long Instruction Word } \\ \text { VPN } & \text { Virtual Private Network; private network realized on } \\ & \text { a shared network infrastructure by means of crypto- } \\ & \text { graphy } \\ \text { Xilinx } & \text { Vendor of }>\text { CPLDs and }>\text { FPGAs } \\ \text { XOR } & \text { exclusive-or (Boolean operation) } \\ \text { XU } & \text { - XOR Unit }\end{array}$




\section{Bibliography}

[1] Record-Breaking DES Key Search Completed. July 1998. http://www.cryptography.com/resources/whitepapers/DES.html.

[2] Project DES. January 1999. http://www.distributed.net/des/.

[3] Marta Dinata A., Sarwono, and Sigit. OpenCores IDEA Core. Sep 2001. http://www.opencores.org/projects/idea/.

[4] Advanced Micro Devices, Inc. AMD 29000 Family Databook. 1987.

[5] I Putu Gede Al, Rudi Harianta, and Hendro Suryadi. OpenCores TwoFish Core. Feb 2002. http://www.opencores.org/projects/twofish_team/.

[6] Alliance for Telecommuncations Industry Solutions. ATIS HomePage. 2002. http://www.atis.org.

[7] Altera Corporation. APEX 20K Devices. 2002. http://www.altera.com/products/devices/apex/apx-index.html.

[8] Altera Corporation. Corporate Web Site. 2002. http://www.altera.com.

[9] Amphion Semiconductor Ltd. Corporate Web Site. 2001. http://www.amphion.com.

[10] Amphion Semiconductor Ltd. CS5210-40 High Performance AES Encryption Cores Product Information. 2001. http://www.amphion.com/acrobat/DS5210-40.pdf. 
[11] Amphion Semiconductor Ltd. CS5210-40 High Performance AES Decryption Cores Product Information. 2002. http://www.amphion.com/acrobat/DS5250-80.pdf.

[12] A. O. L. Atkin and F. Morain. Elliptic Curves and Primality Proving. Math. Comp., 61:203:29-68, July 1993.

[13] Dario Benedetto, Emanuele Caglioti, and Vittorio Loreto. Language Trees and Zipping. Physical Review Letters, 88, January 2002. http://prl.aps.org/.

[14] T. Beth and D. Gollmann. Algorithm Engineering for Public Key Algorithms. IEEE Journal on Selected Areas in Communications, 7 no. 4:458-466, 1989.

[15] Broadcom Corporation. BCM5801 Cryptographic Processor Data Sheet. 2002. http://www.broadcom.com/cgi-bin/access/new_request.cgi?file=5801-5805-5820-SRL101-R.pdf;rt=1 .

[16] Broadcom Corporation. BCM5801 Cryptographic Processor Product Brief. 2002. http://www.broadcom.com/pbs/BCM5801.pdf.

[17] Broadcom Corporation. BCM5802 Cryptographic Processor Data Sheet. 2002. http://www.broadcom.com/cgi-bin/access/new_request.cgi?file=5802-DS01-R.pdf;rt=1 .

[18] Broadcom Corporation. BCM5802 Cryptographic Processor Product Brief. 2002. http://www.broadcom.com/pbs/BCM5801.pdf.

[19] Broadcom Corporation. BCM5805 Security Processor Data Sheet. 2002. http://www.broadcom.com/cgi-bin/access/new request.cgi?file $=5805-\mathrm{DS} 03-\mathrm{R} . \mathrm{pdf} ; \mathrm{rt}=1$.

[20] Broadcom Corporation. BCM5805 Security Processor Product Brief. 2002. http://www.broadcom.com/pbs/BCM5805.pdf.

[21] Broadcom Corporation. BCM5820 E-Commerce Processor Data Sheet. 2002. http://www.broadcom.com/cgi-bin/access/new_request.cgi?file=5820-DS03-R.pdf;rt=1 . 
[22] Broadcom Corporation. BCM5820 E-Commerce Processor Product Brief. 2002. http://www.broadcom.com/pbs/BCM5820.pdf.

[23] Broadcom Corporation. BCM5821 E-Commerce Processor Product Brief. 2002. http://www.broadcom.com/pbs/BCM5821.pdf.

[24] Broadcom Corporation. BCM5840 Gigabit Security Processor Product Brief. 2002. http://www.broadcom.com/pbs/BCM5840.pdf.

[25] Broadcom Corporation. Corporate Web Site. 2002. http://www.broadcom.com.

[26] Ronald H. Brown, Mary L. Good, and Arati Prabhakar. Data Encryption Standard (DES) (FIPS 46-2). Federal Information Processing Standards Publication (FIPS), Dec 1993. http://www.itl.nist.gov/fipspubs/fip46-2.html (initial version from Jan 15, 1977).

[27] Ronald H. Brown and Arati Prabhakar. FIPS180-1: Secure Hash Standard (SHA). Federal Information Processing Standards Publication (FIPS), May 1993. http://www.itl.nist.gov/fipspubs/fip1801.htm.

[28] Ronald H. Brown and Arati Prabhakar. Security Requirements for Cryptographic Modules (FIPS 140-1). Federal Information Processing Standards Publication (FIPS), Jan 1994. http://www.itl.nist.gov/fipspubs/fip140-1.htm (initial version from Apr 14, 1982).

[29] Chris Caldwell. Finding primes \& proving primality, 2002. http://www.utm.edu/research/primes/prove/index.html.

[30] C. M. Campbell. Design and Secification of Cryptographic Capabilities. IEEE Cmputer Society Magazine, 16(6):15-19, November 1978.

[31] Lakshmi N. Chakrapani, Krishna V. Palem, and Weng Fai Wong. Triceps: Enhancing the Trimaran Compiler Infrastructure for Strong/ARM Code Generation. 2001. http://www.crest.gatech.edu/publications/codegen.zip. 
[32] Intel Corporation. Intel 82801 BA I/O Controller Hub 2 (ICH2) and Intel 82801BAM I/O Controller Hub 2 Mobile (ICH2-M). October 2000. Document Number 290687-002.

[33] Intel Corporation. Intel 850 Chipset Family: 82850/82850E Memory Controller Hub. May 2002. Document Number 290691-002.

[34] Intel Corporation. Intel Architecture for Applied Computing. 2002. http://developer.intel.com/design/intarch/.

[35] Intel Corporation. Intel Pentium 4 Processor with 512-KB L2 Cache on 0.13 Micro Process at $2 \mathrm{GHz}, 2.20 \mathrm{GHz}, 2.26 \mathrm{GHz}, 2.40 \mathrm{GHz}$ and 2.53 GHz. May 2002. Document Number 298643-003.

[36] Corrent Corporation. Corporate Web Site. 2002. http://www.corrent.com.

[37] Corrent Corporation. General Product Information. 2002. http://www.corrent.com/products.phtml.

[38] R. Crandall and C. Pomerance. Prime Numbers: A Computational Perspective. Springer-Verlag, New York, 2001. ISBN 0-387-947779.

[39] J. Daemen and V. Rijmen. The block cipher Rijndael, 2000. LNCS1820, Eds: J.-J. Quisquater and B. Schneier.

[40] J. Daemen and V. Rijmen. Advanced Encryption Standard (AES) (FIPS 197). Technical report, Katholijke Universiteit Leuven / ESAT, Nov 2001. http://csrc.nist.gov/publications/fips/fips197/fips-197.pdf.

[41] William M. Daley and Raymond G. Kammer. Fips186-2: Digital signature standard. Federal Information Processing Standards Publication (FIPS), Jan 2000. http://csrc.nist.gov/publications/fips/fips1862/fips186-2.pdf.

[42] D. W. Davies and G. I. P. Parkin. The Average Size of the Key Stream in Output Feedback Encipherment. Advances in Cryptology: Proceedings of Crypto 82, pages 97-98, 1983. 
[43] D. W. Davies and G. I. P. Parkin. The Average Size of the Key Stream in Output Feedback Encipherment. Cryptogprahy, Proceedings of the Workshop on Cryptography, Burg Feuerstein, Germany, March 29-April 2, 1982, pages 263-279, 1983.

[44] W. Diffie and M.E. Hellman. Privacy and Authentication: An Introduction to Cryptography. Proceedings of the IEEE, 67(3):397-427, March 1979.

[45] Dino Oliva. Efficient Implementation of the AES/Rijndael Algorithm. 2001. (internal paper).

[46] Dino Oliva and Nevin Heintze and Rainer Buchty. Straight-forward Implementation of the AES/Rijndael Algorithm. Sep 2001. (internal paper).

[47] Brian Gladman et al. AES Algorithm Efficiency. Feb 1999. http://fp.gladman.plus.com/cryptography technology/aes/.

[48] J. Gait. A New Nonlinear Pseudorandom Number Generator. IEEE Transactions on Software Engineering, SE-3(5):359-363, September 1977.

[49] P. Gaundry, F. Hess, and N. Smart. Constructive and Destructive Facets of Weil Descent on Elliptic Curves. January 2000. http://www.hpl.hp.com/techreports/2000/HPL-2000-10.html.

[50] Heise Online-Redaktion (wst). Zip-Algorithmus identifiziert Autoren. Heise Newsticker, January 2002. http://www.heise.de/newsticker/data/wst-28.01.02-003/.

[51] M.E. Hellman. On DES-Based Synchronous Encryption. 1980.

[52] John L. Hennessy and David A. Patterson. Morgan Kaufmann Publishers Inc., 1996. 2nd Edition.

[53] Hifn Inc. 7711 Encryption Processor Data Sheet. 2002. http://www.hifn.com/docs/a/DS-0001-04-7711.pdf.

[54] Hifn Inc. 7751 Encryption Processor Data Sheet. 2002. http://www.hifn.com/docs/a/DS-0013-03-7751.pdf. 
[55] Hifn Inc. 7811 Network Security Processor Data Sheet. 2002. http://www.hifn.com/docs/a/DS-0018-02-7811.pdf.

[56] Hifn Inc. 7814/7851/7854 Network Security Processors Device Specifications. 2002. http://www.hifn.com/docs/a/DS-0030-04-78147851-7854-Device-Specification.pdf.

[57] Hifn Inc. 7901 Network Security Processor Data Sheet. 2002. http://www.hifn.com/docs/a/DS-0023-01-7901.pdf.

[58] Hifn Inc. 7902 Network Security Processor Data Sheet. 2002. http://www.hifn.com/docs/a/DS-0040-00-7902.pdf.

[59] Hifn Inc. 7951 Network Security Processor Data Sheet. 2002. http://www.hifn.com/docs/a/DS-0028-02-7951.pdf.

[60] Hifn Inc. 8065/8165 Secure Session Processor Product Information. 2002. http://www.hifn.com/docs/HIPPII-8065-8165.pdf.

[61] Hifn Inc. 8154 Security Processor Product Information. 2002. http://www.hifn.com/docs/HIPPII-8154.pdf.

[62] Hifn Inc. 8165 Secure Session Processor Short Data Sheet. 2002. http://www.hifn.com/docs/a/DS-0049-B-8165-Short-DataSheet.pdf.

[63] Hifn Inc. Corporate Web Site. 2002. http://www.hifn.com.

[64] Renato Iannella. Digital Rights Management (DRM) Architectures. D-Lib Magazine, 7.6, June 2001. ISSB 1082-9873, http://www.dlib.org/dlib/june01/iannella/06iannella.html.

[65] Xilinx Inc. Synopsys FPGA Compiler II. 2002.

http://www.xilinx.com/xlnx/xil_prodcat_product.jsp?title=synopsys_fpga_express.

[66] Intel Corporation. Intel Itanium Processor Family Home. 2002. http://developer.intel.com/design/itanium.

[67] Jüri Pöldre. Cryptoprocessor PLD001 (Master Thesis). June 1998. 
[68] R. R. Jueneman. Analysis of Certain Aspects of Output-Feedback Mode. Advances in Cryptology: Proceedings of Crypto 82, pages 99-127, 1983.

[69] S. T. Kent. Encryption-Based Protection Protocols for Interactive User-Computer Communications. MIT/LCS/TR-162, May 1976.

[70] Philip M. Klutznick and Ernest Ambler. DES Modes of Operation (FIPS 81). Federal Information Processing Standards Publication (FIPS), Dec 1980. http://www.itl.nist.gov/fipspubs/fip81.htm.

[71] S. Shrivastava L. Gao and G. Sobelman. Elliptic curve scalar multiplier design using FPGAs. Workshop on Cryptographic Hardware and Embedded Systems (CHES 99), LNCS 1717, August 1999.

[72] X. Lai. Detailed description and a software implementation of the ipes cipher. Nov 1991. unpublished paper.

[73] X. Lai. On the design and security of block ciphers. ETH Series in Information Processing, 1, 1992.

[74] X. Lai, J. Massey, and S. Murphy. Markov ciphers and differential cryptanalysis. Advances in Cryptology - EUROCRYPT 91 Proceedings, pages 17-38, 1991.

[75] K. Leung, K. Ma, W. Wong, and P. Leong. FPGA implementation fo a microcoded elliptic curve cryptographic processor. Eight Annual IEEE Symposium on Field-Programmable Custom Computing Machines (FCCM 00), 2000.

[76] J. Lopez and R. Dahab. Fast multiplication on elliptic curves ofer $G F\left(2^{m}\right)$ without precomputation. Workshop on Cryptographic Hardware and Embedded Systems (CHES 99), LNCS 1717, August 1999.

[77] Microsoft Corporation. Technology \& Tools: Digital Rights Management. December 2001. http://www.microsoft.com/windows/windowsmedia/drm.asp.

[78] National Institute of Standards and Technology. Recommended Elliptic Curves for Federal Government Use, May 1999. http://csrc.nist.gov/encrytion. 
[79] NetOctave, Inc. NSP2000 Security Processor. 2001. http://www.chipcenter.com/networking/images/prod/prod237b.pdf.

[80] NetOctave, Inc. NSP2000-SSL Security Accelerator. 2001. http://www.netoctave.com/netoctave.asp?template=products $\&-$ section $=$ ssl.

[81] NetOctave, Inc. NSP3000-IPsec Security Accelerator. 2001. http://www.netoctave.com/netoctave.asp?template=products \&section=ipsec.

[82] NetOctave, Inc. NSP3200 Security Processor. 2001. http://www.netoctave.com/downloads/NSP3200SecurityProcessor.pdf.

[83] NetOctave, Inc. NSP3200 Security Processor. 2001. http://www.netoctave.com/downloads/FlowThrough_Security_Architecture_WP.pdf.

[84] Netoctave, Inc. Corporate Web Site. 2002. http://www.netoctave.com/.

[85] Open Software Foundation (OSF). OSF Homepage. 2002. http://www.osf.org.

[86] OpenCores.org. Project Website. 2002. http://www.opencores.org/.

[87] G. Orlando and C. Paar. A high performance elliptic curve processor for $G F\left(2^{m}\right)$. Workshop on Cryptographic Hardware and Embedded Systems (CHES 2000), LNCS 1865, August 2000.

[88] C. Paar, P. Fleischmann, and P. Soria-Rodriguez. Fast Arithmetic for Public Key Algorithms in Glaois Fields with Composite Exponents. IEEE Transactions on Computers, 48:1025-1034, October 1999.

[89] Peter N. Glaskowsky. At Least One Chip Niche Is Secure. Microprocessor Report, Feb 2001. http://www.corrent.com/pdf/Corrent_awardarticle_reprint.pdf.

[90] Peter N. Glaskowsky. Cahners In-Stat/MDR Announcement. Feb 2001. http://www.corrent.com/pdf/MPR_press_release.pdf. 
[91] R. Rivest. RFC1186: The MD4 Message-Digest Algorithm. October 1990. http://www.ietf.org/rfc/rfc1186.txt.

[92] R. Rivest. The MD4 message digest algorithm. Advances in Cryptology - CRYPTO '90 Proceedings, pages 303-311, 1991.

[93] R. Rivest. RFC1312: The MD5 Message-Digest Algorithm, April 1992. http://www.ietf.org/rfc/rfc1321.txt.

[94] R.L. Rivest. The RC5 Encryption Algorithm. Dr. Dobb's Journal, 20(1):146-148, Jan 1995.

[95] R.L. Rivest. The RC5 Encryption Algorithm. K.U. Leuven Workshop on Cryptographic Algorithms, 1995.

[96] Ronald R. Rivest, M.J.B. Robshaw, R. Sidney, and Y.L. Yin. The RC6 ${ }^{T M}$ Block Cipher. August 1998. http://www.rsasecurity.com/rsalabs/rc6/.

[97] Ronald R. Rivest, M.J.B. Robshaw, and Y.L. Yin. The Case for RC6 as the AES. May 2000. http://www.rsasecurity.com/rsalabs/rc6/.

[98] M. Rosner. Elliptic curve cryptosystems on reconfigurable hardware. ECE Dept, Worcester Polytechnic Institute, Worcester, USA, May 1998. Master's Thesis.

[99] Ray Savarda. Next Generation Network Security Processors: Optimal Design and Integration with Network Processors. 2001.

http://www.netoctave.com/downloads/NextGen_SecurityProcessors.pdf.

[100] Ray Savarda. Next Generation Network Security Processors: Optimal Design and Integration with Network Processors. Communications Design Conference 2001, 2001.

[101] Bruce Schneier. 13.9: IDEA. Angewandte Kryptographie: Protokolle, Algorithmen und Sourcecode in C, pages 370-377, 1996. ISBN 3-89319-854-7.

[102] Bruce Schneier. 24.12: Pretty Good Privacy (PGP). Angewandte Kryptographie, pages 664-667, 1996. ISBN 3-89319-854-7. 
[103] Bruce Schneier. 9.10 - Weitere Modi für Blockchiffrierung. Angewandte Kryptographie, pages 244-246, 1996. ISBN 3-89319-854-7.

[104] Bruce Schneier. 9.3 - Cipher Block Chaining. Angewandte Kryptographie, pages 227-232, 1996. ISBN 3-89319-854-7.

[105] Bruce Schneier. Algorithmenarten und Betriebsmodi. Angewandte Kryptographie, pages 223-250, 1996. ISBN 3-89319-854-7.

[106] Bruce Schneier. Angewandte Kryptographie: Protokolle, Algorithmen und Sourcecode in C. Addison Wesley, 1996. ISBN 3-89319854-7.

[107] Bruce Schneier. Einweg-Hashfunktionen: MD5. Angewandte Kryptographie: Protokolle, Algorithmen und Sourcecode in C, pages 498503, 1996. ISBN 3-89319-854-7.

[108] Bruce Schneier. Einweg-Hashfunktionen: SHA. Angewandte Kryptographie: Protokolle, Algorithmen und Sourcecode in C, pages 504507, 1996. ISBN 3-89319-854-7.

[109] Bruce Schneier. Noch mehr Blockchiffrierungen: RC5. Angewandte Kryptographie: Protokolle, Algorithmen und Sourcecode in C, pages 397-399, 1996. ISBN 3-89319-854-7.

[110] Bruce Schneier, John Kelsey, Doug Whiting, David Wagner, Chris Hall, and Niels Ferguson. AES Performance Comparisons. Oct 2000. http://csrc.nist.gov/encryption/aes/round1/conf2/Schneier.pdf.

[111] R. Schroeppel, H. Orman, S. O’Malley, and O. Spatscheck. Fast Key Exchange with Elliptic Curve Systems. Advance in Cryptography, Crypto 95, LNCS 963, 1995.

[112] SecuCore Consulting Services. SecuCore AES/Rijndael Core. 2001. http://www.secucore.com/secucore_aes.pdf.

[113] SecuCore Consulting Services. SecuCore DES/3DES Core. 2001. http://www.secucore.com/secucore_des.pdf.

[114] SecuCore Consulting Services. SecuCore SHA-1/MD5/HMAC Core. 2001. http://www.secucore.com/secucore_hmac.pdf. 
[115] SecuCore Consulting Services. SecuCore SHA-256 Core. 2001. http://www.secucore.com/secucore_sha256.pdf.

[116] SecuCore Consulting Services. Corporate Web Site. 2002. http://www.secucore.com/.

[117] L. Song and K. K. Parhi. Low-energy digit-serial/parallel finite field multipliers. Journal of VLSI Signal Processing Systems, 2 no. 22:117, 1997.

[118] Synopsys, Inc. Corporate Web Site. 2002. http://www.synopsys.com.

[119] Taiwan Semiconductor Manufacturing Company Ltd. Corporate Web Site. 2002. http://www.tsmc.com.

[120] Paulus Tamba. OpenCores Crypto Accelerator. Sep 2001. http://www.opencores.org/projects/crypto/.

[121] Telecom Glossary 2000. Cryptanalysis, 2000. http://www.its.bldrdoc.gov/fs-1037/dir-009/_1343.htm.

[122] Telecom Glossary 2000. Cryptography, 2000. http://www.its.bldrdoc.gov/projects/t1glossary2000/_cryptography.html.

[123] Telecom Glossary 2000. Cryptology, 2000. http://www.its.bldrdoc.gov/projects/t1glossary2000/_cryptology.html.

[124] The FreeS/WAN Project Team. FreeS/WAN Project. 2002. http://www.freeswan.org/.

[125] United Microelectronics Corp. Corporate Web Site. 2001. http://www.umc.com.

[126] United States Patent and Trademark Office. Official Gazette of the United States Patent and Trademark Office. May 1975. 934 O.G. 452.

[127] United States Patent and Trademark Office. Official Gazette of the United States Patent and Trademark Office. Aug 1976. 949 O.G. 1717. 
[128] Rudolf Usselmann. OpenCores DES Core. Sep 2001. http://www.opencores.org/projects/des/.

[129] A. Wiles. Modular Elliptic Curves and Fermat's last theorem. Ann. Math., 141:3:443-551, 1995.

[130] H. Wu. Low complexity bit-parallel finite field arithmetic using polynomial basis. Workshop on Cryptographic Hardware and Embedded Systems (CHES 99), LNCS 1717, August 1999.

[131] Lisa Wu, Chris Weaver, and Todd Austin. Cryptomaniac: A fast flexible architectore for secure communication. In 28th Annual International Symposium on Computer Architecture (ISCA 2001), June 2001.

[132] Xilinx, Inc. Corporate Web Site. 2002. http://www.xilinx.com.

[133] Xilinx, Inc. Virtex-II Pro Platform FPGAs. 2002.

http://www.xilinx.com/xlnx/xil_prodcat_landingpage.jsp?title=Virtex-II+Pro+FPGAs.

[134] Chi Lap Yuen. OpenCores RSA RC4 Core. Apr 2002.

http://www.opencores.org/projects/rsa-rc4/.

[135] Chi Lap Yuen and Kurt Ting. OpenCores NIST Secure Hash Algorithm 256 Core. Apr 2002.

http://www.opencores.org/projects/sha256/.

[136] Made Yusadana. OpenCores AES/Rijndael Core. Feb 2002. http://www.opencores.org/projects/rijndael/.

[137] Zilog, Inc. Z80 Product Details. 2002. http://www.zilog.com/products/parts.asp?BusinessLineID=274.

[138] P.R. Zimmermann. PGP Source Code and Internals. 1995.

[139] P.R. Zimmermann. The Official PGP User's Guide. 1995.

[140] Zyfer, Inc. Corporate Web Site. 2002. http://www.zyfer.com.

[141] Zyfer, Inc. SKP-100 Gigabit Network Security Processor. 2002. http://www.zyfer.com/products/SKP-100_Data_Sheet.pdf. 
Index

- Symbols -

3DES, 22

$$
-\mathbf{A}-
$$

Advanced Encryption Standard, see AES

AES, 22, 30, 76, 77

algorithm analysis, 32

column mixing, 34

data chunk size, 30

key addition, 33

key scheduling, 31

row shifting, 33

substitution, 33

AES/Rijndael, see AES

approving data by a third person, 5

architecture summary, 90

asymmetric encryption, 3,12

audio streams, 7

authentication, 2, 3

$$
-\mathbf{B} \text { - }
$$

bandwidth, 64

block cipher, 3
- C -

Caesar chiffre, 11

CBC, 13, 14

CFB, 13,16

chaining variables, 36,40

cipher algorithm, 11

Cipher Block Chaining, see CBC

Cipher Feedback Mode, see CFB

cipher key, 6

CM, 13, 18

conditional branching, 71

control unit, 74

cryptanalysis, 1

cryptography, 1

cryptology, 1, 2

CryptoManiac, 114

CRYPTONITE

address generation, 66

addressing mode, 66

addressing modes, 81

AGU, 66, 81

ALU, 74

ALU-common control encoding, 151

arithmetic instructions, 145 
AU, 66, 74, 76

AU command encoding, 153

bitmux unit, 79

control instructions, 142

control unit, 71

CU command encoding, 151

DES unit, 68, 77

design goals, 64

DIO, 66, 85

external access, 69

fold operations, 79

Hold input, 70

immediate value support, 141,150

instruction word, 74, 149

instruction word format, 74

LAR, 82

linking of arithmetic units, 80

loop support, 73

lower64 operation, 77

memory instructions, 142

MU, 66, 81

MU command encoding, 155

overview, 66, 74

pipeline, 68

Ready flag, 69

register file, 74, 74

register naming convention, 157

S-Box support, 84

sign flag, 73

special functions, 77

summary, 90

swap operation, 79

swrt operation, 79

synchronous transfer, 70

technology limitations, 64 timing constraints, 64

upper64 operation, 77

XOR instructions, 145

XOR unit, 74, 80

XU command encoding, 153

zero flag, 73

CRYPTONITE

foldb32 operation, 79

foldb64 operation, 79

foldw64 operation, 79

- D -

data distribution, 4

Data Encryption Standard, see DES

DES, 22, 30, 77, 87, 102

data chunk size, 30

decryption, 25

encryption, 25

expansion permutation, 25

initial approach, 87

key compression, 25

modes of operation, 23

monolithic operations, 88

P-Box permutation, 25

pipelined, 88

round key generation, 24

S-Box, 25

single operations, 88

design goals, 64

Digital Rights Management, see DRM

DIO, 81

distributed secret sharing, 5

DRM, 4

$$
\text { - E - }
$$

ECB, 13, 13 
Electronic Codebook Mode, see ECM

electronic voting, see secret voting

elliptic curve equations, 6

elliptic curve processors, 6

exchanging data through a trusted third party, 5

$$
-\mathbf{F}-
$$

FreeS/WAN, 5

$$
-\mathbf{G}-
$$

general purpose architectures, 65 general purpose processor, 102

$$
-\mathbf{H}-
$$

hardware solutions, 102

hash, 11

hash alagorithm, 13

hash algorithm, 11

$$
\text { - I - }
$$

IDEA, 22, 43, 73

algorithm analysis, 45

architectural requirements, 48

round key generation, 44

identity fraud, 4

immediate exchange of secrets, 5

immediate signing, 5

intranet, 5

IP cores, 102

IPES, see IDEA

IPSEC, 5

$$
-\mathbf{K}-
$$

key generation, 5

key scheduling, see round key generation key spanning, see round key generation

$$
-\mathbf{L} \text { - }
$$

LAR, 82

linear addressing, 71

loop support, 71

$$
-\mathrm{M}-
$$

MD4, 22, 39

MD4 Message Digest Algorithm, see MD4

MD5, 22, 36

chaining variables, 36

hash generation, 38

non-linear functions, 37, 39

rounds, 36, 38

MD5 Message Digest Algorithm, see MD5

message authentication, 13

Modes of Operation, 13

CBC, 13,14

CFB, 13,16

CM, 13,18

ECB, 13, 13

OFB, 13, 17

modulo addressing, 82

multimedia streams, 7

$$
-\mathbf{O} \text { - }
$$

OFB, 13, 17

one-to-many broadcast, 2

operation mode, see Mdes of Operation 13

Output Feedback Mode, see OFB

$$
-\mathbf{P} \text { - }
$$

Personal Identification Number, see PIN 
PES, see IDEA

PIN, 3

point-to-point connection, 2

prime number generation, 6

private key, 12

programmable solutions, 114

public key, 12

public key algorithm, 12

$$
-\mathbf{R}-
$$

$\mathrm{RC} 5,48$

RC5 Block Cipher, see RC5

RC6, 22, 48

RC6 Block Cipher, see RC6

real-time requirements, 7

round, 6

round key generation, 6

AES/Rijndael, 30, 31

DES, 28

IDEA, 44

RC6, 50

$$
-\mathbf{S}-
$$

S-Box addressing, 84

S-Box support, 84

secret voting, 5

secure data transfer, 3

SHA, 22, 39, 40

SHA-1 Secure Hash Algorith, see

SHA

single-cycle access, 64

single-cycle execution, 64

stream cipher, 3

symmetric encryption, 3,12

$$
-\mathbf{T} \text { - }
$$

table lookup acceleration, 84

TAN, 3 technology limitations, 64

throughput, 64

timing constraints, 64

Transaction Number, see TAN

Triple DES, see 3DES

tunneling, 7

$$
-\mathrm{V}-
$$

video transmission, 7

Virtual Private Network, see VPN

VPN, 4, 7 